\title{
2D/2D heterostructured photocatalyst: Rational design for energy and environmental applications
}

\author{
Huilin Hou ${ }^{1,2}$, Xiangkang Zeng ${ }^{2}$ and Xiwang Zhang ${ }^{2 *}$
}

\begin{abstract}
Two-dimensional/two-dimensional (2D/2D) hybrid nanomaterials have triggered extensive research in the photocatalytic field. The construction of emerging 2D/2D heterostructures can generate many intriguing advantages in exploring high-performance photocatalysts, mainly including preferable dimensionality design allowing large contact interface area, integrated merits of each $2 \mathrm{D}$ component and rapid charge separation by the heterojunction effect. Herein, we provide a comprehensive review of the recent progress on the fundamental aspects, general synthesis strategies (in situ growth and ex situ assembly) of 2D/2D heterostructured photocatalysts and highlight their applications in the fields of hydrogen evolution, $\mathrm{CO}_{2}$ reduction and removal of pollutants. Furthermore, the perspectives on the remaining challenges and future opportunities regarding the development of 2D/2D heterostructure photocatalysts are also presented.
\end{abstract}

Keywords: 2D/2D heterojunction, photocatalyst, hydrogen evolution, $\mathrm{CO}_{2}$ reduction, pollutant removal

\section{INTRODUCTION}

Two-dimensional (2D) materials have been deemed as rapidly rising stars in materials science, because they display a variety of extraordinary physicochemical and mechanical properties different from their bulk counterparts, such as large surface-to-volume ratio, high transparency and good flexibility [1-5]. Since the groundbreaking discovery of mono-layered graphene [6], plenty of newly developed 2D materials have been applied in many fields including energy storage [7], electrocatalysis [8], sensing [9], and photocatalysis [10]. Particularly, the utilization of $2 \mathrm{D}$ materials for photocatalysis has triggered considerable interest, and promptly become one of the hottest research topics [11-13]. It has been demonstrated that $2 \mathrm{D}$ material-based photocatalysts are expected to offer intriguing features such as high specific surface areas, porous structures, good conductivity and superior electron mobility, rich options of host-guest species and abundant surface active sites, which are beneficial to photocatalytic reactions [14,15]. Moreover, heterostructures of 2D materials further offer an effective way for enhancing light absorption, and promoting the separation and transfer of photogenerated charge carriers [16-19]. Typically, it is well-known that the appropriate band alignments thermodynamically between the components should be firstly required in heterostructure design. By coupling the different band structures in various materials, the light responsive range is broadened while the charge separation and transportation is synchronously promoted, leading to enhanced photocatalytic activities [20-23]. However, besides the band structure matching, the interface contact, governed by the dimensionality of each component, is also critically vital for the smooth transfer of the photogenerated charge carriers. In regard to the dimensionality, 2D based heterostructures composed of two components can be classified into $0 \mathrm{D} / 2 \mathrm{D}, 1 \mathrm{D} / 2 \mathrm{D}$ and $2 \mathrm{D} / 2 \mathrm{D}$ heterostructures with three different types of interfacial contacts as illustrated in Fig. 1. Distinctly, the face-to-face stacking of 2D/2D heterostructures has the largest interfacial contact area and strongest interactions, which enables relatively better coupling hetero-interfaces compared with $0 \mathrm{D} / 2 \mathrm{D}$ and $1 \mathrm{D} / 2 \mathrm{D}$ heterostructures $[10,15]$. The large contact area and strong interactions can also facilitate the transfer and separation of photogenerated electron-hole pairs, thus contributing to high photocatalytic performance. Profiting from these features, 2D/2D photocatalyst can display enormous prospects and a large number of related studies

\footnotetext{
${ }^{1}$ Institute of Materials, Ningbo University of Technology, Ningbo 315016, China

${ }^{2}$ Department of Chemical Engineering, Monash University, Clayton, VIC, 3800, Australia

* Corresponding author (email: xiwang.zhang@monash.edu)
} 


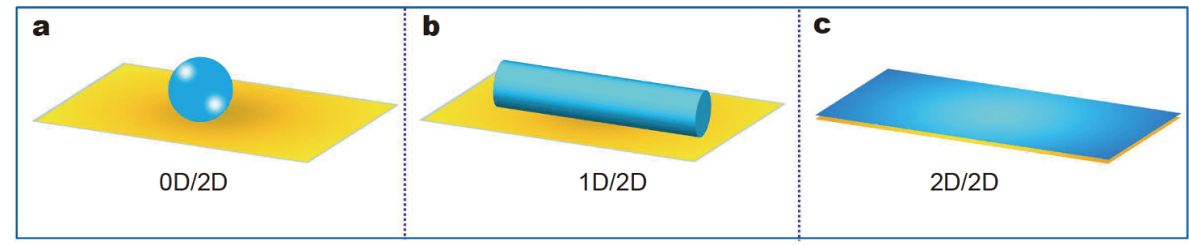

Figure 1 Schematic illustration of 2D based heterostructures in regard to the dimensionality difference.

have been reported in recent years. Although the research progress of $2 \mathrm{D} / 2 \mathrm{D}$ heterostructure photocatalysts has been mentioned in some excellent reviews on $2 \mathrm{D}$ based photocatalysts $[10,11,24,25]$, a thoroughly comprehensive review to specifically highlight this subject has yet been reported. Thus, it is highly desirable and urgent to present a comprehensive review on this subject to provide a better understanding of the state-of-the-art progress in this research field as well as promote the further development of $2 \mathrm{D} / 2 \mathrm{D}$ heterostructured photocatalysts.

Here, we present a comprehensive review to summarize the advanced progress of $2 \mathrm{D} / 2 \mathrm{D}$ heterostructure photocatalysts (Fig. 2). It begins with a brief discussion of the fundamental aspects of $2 \mathrm{D} / 2 \mathrm{D}$ photocatalysts. Then, the emerging strategies for designing various $2 \mathrm{D} / 2 \mathrm{D}$ heterostructures are brifly summarized. Subsequently, we highlight the recent progress of $2 \mathrm{D} / 2 \mathrm{D}$ heterostructures for photocatalytic applications, including photocatalytic hydrogen production, carbon dioxide $\left(\mathrm{CO}_{2}\right)$ reduction and the removal of pollutions. Finally, a brief summary of the current the research status and key issues, along with the perspectives of $2 \mathrm{D} / 2 \mathrm{D}$ heterostructures for photocatalysis applications will also be discussed.

\section{FUNDAMENTAL ASPECTS OF 2D/2D HETEROJUNCTION PHOTOCATALYSTS}

\section{The interfacial coupling in 2D/2D photocatalysts}

The activity and stability of the $2 \mathrm{D} / 2 \mathrm{D}$ photocatalysts are highly dependent on the type and quality of the interface in $2 \mathrm{D} / 2 \mathrm{D}$ photocatalysts [26]. Generally, the layers in $2 \mathrm{D}$ material heterostructures can be combined by covalent bonding and van der Waals (vdW) forces [27]. To form a well-defined covalent bonding interface in $2 \mathrm{D} / 2 \mathrm{D}$ photocatalysts, it needs not only the lattice constant matching, but also valence matching on each side of the interface [3,27] (Fig. 3a). In contrast to the covalent bonding interfaces, the vdW forces between the layers in 2D material heterostructures are relatively weak. In addition, the vdW integration does not rely on one-to-one lattice-matching and valence-matching, ultimately en-

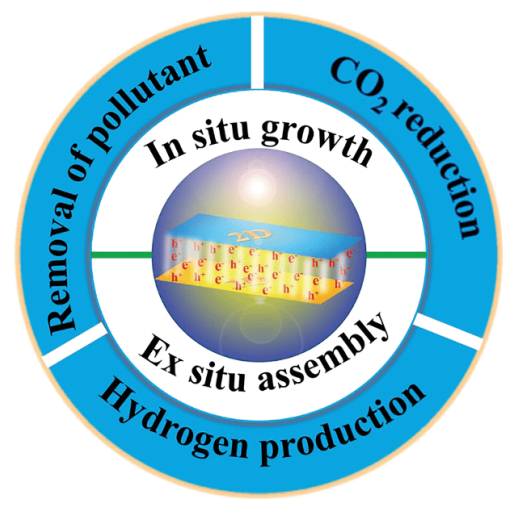

Figure 2 Schematic diagram for the scope of this review.
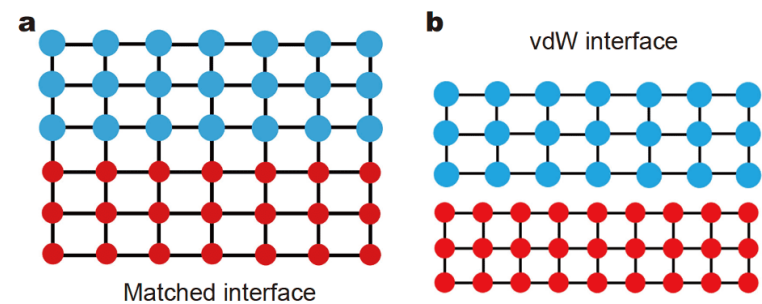

Figure 3 (a) Schematic illustrations of bonded heterostructure interface with a lattice-matched interface. (b) Bonding free atomic structure at a $\mathrm{vdW}$ interface. Reprinted with permission from Ref. [3]. Copyright 2019, Nature.

abling a broader heterostructure phase space (Fig. 3b). Although vdW force is weak, the formed interaction can still mediate various types of coupling across the $2 \mathrm{D} / 2 \mathrm{D}$ interface $[3,27]$. For example, it can redistribute the charges at interfaces to balance the chemical potential, which would result in band bending phenomena in the $2 \mathrm{D} / 2 \mathrm{D}$ photocatalysts. In recent years, the form of $2 \mathrm{D} / 2 \mathrm{D}$ photocatalysts with vdW interface has attracted considerable interests because of its bond-free integration feature.

\section{Characterization of interfaces in 2D/2D photocatalysts}

Besides the interfacial coupling type, the characterization 
of interface is also vital to guarantee its quality in $2 \mathrm{D} / 2 \mathrm{D}$ photocatalysts [26]. To investigate the quality and interaction between the $2 \mathrm{D}$ components, many powerful characterization techniques have been employed, mainly including transmission electron microscopy (TEM), atomic force microscopy (AFM), X-ray photoelectron spectroscopy (XPS), and Kelvin probe force microscopy (KPFM) [26].

Low-magnification TEM is often used to explore the lateral size and roughly measure the thickness of the $2 \mathrm{D}$ nanosheet. However, the high-resolution TEM (HRTEM) is often performed to reveal the interfaces between the $2 \mathrm{D} / 2 \mathrm{D}$ photocatalysts. AFM is an useful technique to accurately measure the thickness of $2 \mathrm{D}$ materials. When it is used to characterize the interface in $2 \mathrm{D} / 2 \mathrm{D}$ materials, it should be switched to a conductive AFM (CAFM) mode, which is a variation of AFM. Then, the CAFM can provide a conductivity mapping to clearly explore the interface between two 2D materials. XPS is a well-known surface-sensitive and spectroscopic technique to identify the elemental composition of a material. Specially, the formation of the interface would cause a change in the electron configuration owing to the interaction between the $2 \mathrm{D}$ materials in $2 \mathrm{D} / 2 \mathrm{D}$ photocatalysts. In this case, XPS can be employed to examine the interaction intensity between the $2 \mathrm{D}$ materials. It has been confirmed that the interfacial interaction between the 2D materials could alter the surface potential of a catalyst. KPFM is considered as a powerful technique to study the surface potential and reveal the interfacial interaction between $2 \mathrm{D}$ materials.

\section{SYNTHESIS METHODS FOR 2D/2D HETEROJUNCTION PHOTOCATALYSTS}

\section{In situ growth of 2D/2D heterojunction photocatalysts}

The in situ growth of 2D/2D heterostructures is normally implemented through the following steps. One of the two $2 \mathrm{D}$ materials is firstly synthesized. Then, another 2D component directly grows on the primarily synthesized 2D substrate through one-step or multi-step conversion approaches, such as wet-chemical synthesis and chemical vapor deposition (CVD) methods. Generally, the wetchemical strategy is one of the most commonly used methods for assembling 2D/2D heterostructures due to the simplicity, low cost and high tunability of composition, mainly including hydrothermal/solvothermal, solution deposition and ion exchange. A large variety of $2 \mathrm{D} /$ 2D heterostructures have been synthesized by this route, which are briefly summarized in Table 1 .
As for the hydrothermal/solvothermal method, the presynthesized 2D materials were immersed in the precursor solution of the second $2 \mathrm{D}$ component to obtain a mixed suspension. The $2 \mathrm{D} / 2 \mathrm{D}$ heterostructures were produced by the hydrothermal or solvothermal treatment of the suspension. For instance, Cao et al. [28] developed a $\mathrm{Ti}_{3} \mathrm{C}_{2} / \mathrm{Bi}_{2} \mathrm{WO}_{6} 2 \mathrm{D} / 2 \mathrm{D}$ heterostructure by an in situ hydrothermal growth of $\mathrm{Bi}_{2} \mathrm{WO}_{6}$ nanosheets on ultrathin $\mathrm{Ti}_{3} \mathrm{C}_{2}$ nanosheets. As shown in Fig. $4 \mathrm{a}$, few-layers ultrathin $\mathrm{Ti}_{3} \mathrm{C}_{2}$ nanosheets were firstly obtained by etching bulk $\mathrm{Ti}_{3} \mathrm{AlC}_{2}$ combining with ultrasonic exfoliation process. Because of the negative potential of $\mathrm{Ti}_{3} \mathrm{C}_{2}, \mathrm{Bi}^{3+} \mathrm{ca}-$ tions could be adsorbed on the $\mathrm{Ti}_{3} \mathrm{C}_{2}$ nanosheet surface through electrostatic attraction in the $\mathrm{Bi}\left(\mathrm{NO}_{3}\right)_{3} / \mathrm{Ti}_{3} \mathrm{C}_{2}$ suspension. Subsequently, $\mathrm{Na}_{2} \mathrm{WO}_{6}$-cetyl trimethyl ammonium bromide (CTAB) mixed solution was added into the above suspension. Finally, $\mathrm{Ti}_{3} \mathrm{C}_{2} / \mathrm{Bi}_{2} \mathrm{WO}_{6} 2 \mathrm{D} / 2 \mathrm{D}$ heterostructure was formed after a hydrothermal treatment. In some other studies, the second 2D component was amorphous after hydrothermal reaction and thus an additional calcination process was often required to crystallize it. For example, Cheng and coworkers [29] firstly obtained $\mathrm{C}_{3} \mathrm{~N}_{4}$ nanosheets by ultrasonic exfoliation of bulk $\mathrm{C}_{3} \mathrm{~N}_{4}$, and then immersed them in $\mathrm{Bi}\left(\mathrm{NO}_{3}\right)_{35} \mathrm{H}_{2} \mathrm{O}$ / $\mathrm{Ti}\left(\mathrm{OC}_{3} \mathrm{H}_{7}\right)_{4} / \mathrm{NaBH}_{4}$ benzyl alcohol solution. The $2 \mathrm{D} / 2 \mathrm{D}$ $\mathrm{C}_{3} \mathrm{~N}_{4} / \mathrm{Bi}_{20} \mathrm{TiO}_{32}$ heterojunctions were obtained by hydrothermal reaction and following calcination (Fig. 4b).

Another wet-chemical method for in situ growth of the second 2D component is solution deposition. Similarly, the first $2 \mathrm{D}$ nanosheet is pre-synthesized and then dispersed into some specific solvents to obtain suspension. In this process, one or more reactant ions are firstly adsorbed on the surface of the pre-synthesized 2D substrate through electrostatic attraction. Then, other reaction solutions are added into the mixed suspension under stirring and kept for some times to generate the second $2 \mathrm{D}$ component through chemical precipitation reaction. Compared with the hydrothermal or solvothermal process, the whole process of this route is at room temperature and atmospheric pressure, but only several specific $2 \mathrm{D} / 2 \mathrm{D}$ heterostructures can be prepared by this strategy. Xia et al. [30] used the solution deposition method for in situ growth of $\mathrm{MnO}_{2}$ nanosheets on the surface of $\mathrm{g}-\mathrm{C}_{3} \mathrm{~N}_{4}$ nanolayers. As shown in Fig. $5 \mathrm{a}$, the g$\mathrm{C}_{3} \mathrm{~N}_{4}$ nanosheets were obtained after exfoliation, which possessed a large number of defects, such as dangling bonds, hydroxyl groups, and exposed lone pair electrons from $\mathrm{N}$ atoms. These defects promoted the adsorption of $\mathrm{Mn}^{2+}$ on the surface of $\mathrm{g}-\mathrm{C}_{3} \mathrm{~N}_{4}$ nanosheets. Then, $\mathrm{Mn}^{2+}$ would be gradually oxidized to generate $\mathrm{MnO}_{2}$ na- 
Table 1 In situ growth of 2D/2D heterostructures through wet chemical method

\begin{tabular}{|c|c|c|c|}
\hline 2D/2D heterojunction & Pre-synthesized 2D component/method & Growth method for the second 2D component & References \\
\hline $\mathrm{g}-\mathrm{C}_{3} \mathrm{~N}_{4}-\mathrm{GO} / \mathrm{MoS}_{2}$ & g- $\mathrm{C}_{3} \mathrm{~N}_{4}$-GO/Hydrothermal & Hydrothermal & [36] \\
\hline $\mathrm{TiO}_{2} / \mathrm{GO}$ & GO/Modified Hummer's method & Solvothermal & [37] \\
\hline $\mathrm{C}_{3} \mathrm{~N}_{4} / \mathrm{Bi}_{20} \mathrm{TiO}_{32}$ & $\mathrm{C}_{3} \mathrm{~N}_{4} /$ Ultrasonic exfoliation & Hydrothermal + Calcination & [26] \\
\hline $\mathrm{CdS} / \mathrm{rGO}$ & GO/Modified Hummer's method & Hydrothermal + Hydrazine hydrate reduction & [38] \\
\hline GO/Mesoporous $\mathrm{TiO}_{2}$ & GO/Modified Hummer's method & Hydrothermal + Calcination & [39] \\
\hline $\mathrm{MoS}_{2} / \mathrm{TiO}_{2}$ & $\mathrm{TiO}_{2} /$ Hydrothermal & Hydrothermal & [40] \\
\hline $\mathrm{BiOIO}_{3} / \mathrm{BiOI}$ & $\mathrm{BiOIO}_{3} /$ hydrothermal & Chemical precipitation method & [41] \\
\hline $\mathrm{P}-\mathrm{C}_{3} \mathrm{~N}_{4} / \mathrm{ZnIn}_{2} \mathrm{~S}_{4}$ & P- $\mathrm{C}_{3} \mathrm{~N}_{4} /$ Frozen expansion and post-thermal exfoliation & Hydrothermal & [42] \\
\hline $\mathrm{TiO}_{2} / \mathrm{Bi}_{2} \mathrm{WO}_{6}$ & $\mathrm{TiO}_{2} /$ Hydrothermal & Hydrothermal & [43] \\
\hline $\mathrm{MnO}_{2} / \mathrm{rGO}$ & rGO/Modified Hummers' method + Calcination & Hydrothermal & [44] \\
\hline $\mathrm{BiOBr} / \mathrm{La}_{2} \mathrm{Ti}_{2} \mathrm{O}_{7}$ & $\mathrm{La}_{2} \mathrm{Ti}_{2} \mathrm{O}_{7} /$ Hydrothermal & Chemical precipitation & [45] \\
\hline $\mathrm{BiOCl} / \mathrm{La}_{2} \mathrm{Ti}_{2} \mathrm{O}_{7}$ & $\mathrm{La}_{2} \mathrm{Ti}_{2} \mathrm{O}_{7} /$ Hydrothermal & Chemical precipitation & [46] \\
\hline $\mathrm{BiOI} / \mathrm{g}-\mathrm{C}_{3} \mathrm{~N}_{4}$ & g- $\mathrm{C}_{3} \mathrm{~N}_{4} /$ Ultrasonic exfoliation & Chemical precipitation & [47] \\
\hline $\mathrm{MnO}_{2} / \mathrm{g}-\mathrm{C}_{3} \mathrm{~N}_{4}$ & g- $\mathrm{C}_{3} \mathrm{~N}_{4} /$ Thermal exfoliation & Redox reaction & [48] \\
\hline $\mathrm{MnO}_{2} / \mathrm{g}-\mathrm{C}_{3} \mathrm{~N}_{4}$ & g- $\mathrm{C}_{3} \mathrm{~N}_{4} /$ Nitric acid and hydrogen peroxide exfoliation & Redox reaction & [30] \\
\hline $\mathrm{Bi}_{4} \mathrm{Ti}_{3} \mathrm{O}_{12} / \mathrm{I}-\mathrm{BiOCl}$ & $\mathrm{Bi}_{4} \mathrm{Ti}_{3} \mathrm{O}_{12} /$ Molten salt synthesis & Chemical transformation & [31] \\
\hline $\mathrm{TiO}_{2} / \mathrm{SnS}_{2}$ & $\mathrm{TiO}_{2} /$ Hydrothermal & Hydrothermal & [49] \\
\hline $\mathrm{Bi}_{2} \mathrm{WO}_{6} / \mathrm{RGO} / \mathrm{g}-\mathrm{C}_{3} \mathrm{~N}_{4}$ & rGO/g- $\mathrm{C}_{3} \mathrm{~N}_{4} /$ heat-etchin + Hydrothermal & Hydrothermal & [50] \\
\hline $\mathrm{Ti}_{3} \mathrm{C}_{2} / \mathrm{Bi}_{2} \mathrm{WO}_{6}$ & $\mathrm{Ti}_{3} \mathrm{C}_{2} /$ Ultrasonic exfoliation & Hydrothermal & [28] \\
\hline $\mathrm{N}-\mathrm{ZnO}-\mathrm{g}-\mathrm{C}_{3} \mathrm{~N}_{4}$ & g- $\mathrm{C}_{3} \mathrm{~N}_{4} /$ Ultrasonic exfoliation & Hydrothermal & {$[51]$} \\
\hline $\mathrm{N}-\mathrm{ZnO}-\mathrm{g}-\mathrm{C}_{3} \mathrm{~N}_{4}$ & g- $\mathrm{C}_{3} \mathrm{~N}_{4} /$ Ultrasonic exfoliation & Hydrothermal & {$[52]$} \\
\hline $\mathrm{g}-\mathrm{C}_{3} \mathrm{~N}_{4} / \mathrm{ZnIn}_{2} \mathrm{~S}_{4}$ & g- $\mathrm{C}_{3} \mathrm{~N}_{4} /$ Thermal exfoliation & Surfactant-assisted solvothermal & [53] \\
\hline $\mathrm{SnNb}_{2} \mathrm{O}_{6} / \mathrm{CoFe}-\mathrm{LDH}$ & $\mathrm{SnNb}_{2} \mathrm{O}_{6} /$ Hydrothermal & Hydrothermal & {$[54]$} \\
\hline $\mathrm{BiOI} / \mathrm{BiVO}_{4}$ & $\mathrm{BiOI} /$ Hydrolysis & Anion-exchange & [55] \\
\hline $\mathrm{Cu} / \mathrm{TiO}_{2} @ \mathrm{Ti}_{3} \mathrm{C}_{2} \mathrm{Tx}$ & $\mathrm{Ti}_{3} \mathrm{C}_{2} \mathrm{Tx} /$ Etching & Hydrothermal & [56] \\
\hline $\mathrm{g}-\mathrm{C}_{3} \mathrm{~N}_{4} / \mathrm{NiAl}-\mathrm{LDH}$ & g- $\mathrm{C}_{3} \mathrm{~N}_{4} /$ Ultrasonic exfoliation & Hydrothermal & {$[57]$} \\
\hline $\mathrm{BiOCl} / \mathrm{g}-\mathrm{C}_{3} \mathrm{~N}_{4}$ & g- $\mathrm{C}_{3} \mathrm{~N}_{4} /$ Ultrasonic exfoliation & Hydrothermal & {$[58]$} \\
\hline $\mathrm{Zn}_{3} \mathrm{In}_{2} \mathrm{~S}_{6} / \mathrm{F}-\mathrm{g}-\mathrm{C}_{3} \mathrm{~N}_{4}$ & F-g- $\mathrm{C}_{3} \mathrm{~N}_{4} /$ Hydrothermal & Hydrothermal & [59] \\
\hline $\mathrm{CoMoS}_{2} / \mathrm{rGO} / \mathrm{C}_{3} \mathrm{~N}_{4}$ & $\begin{array}{l}\text { g- }-\mathrm{C}_{3} \mathrm{~N}_{4} / \text { Thermal exfoliation } \\
\text { rGO/Hummer's method and reduction }\end{array}$ & Solvothermal & {$[60]$} \\
\hline $\mathrm{MoS}_{2} / \mathrm{CdS}$ & CdS/Hydrothermal & Hydrothermal & {$[61]$} \\
\hline $\mathrm{g}-\mathrm{C}_{3} \mathrm{~N}_{4} / \mathrm{rGO} / \mathrm{MoS}_{2}$ & $\mathrm{~g}-\mathrm{C}_{3} \mathrm{~N}_{4}-\mathrm{rGO} /$ Pyrolysis & Hydrothermal & {$[62]$} \\
\hline $\mathrm{CuInS}_{2} / \mathrm{SnS}_{2}$ & $\mathrm{SnS}_{2} /$ Hydrothermal & Hydrothermal & {$[63]$} \\
\hline $\mathrm{ZnCr}-\mathrm{CLDH} / \mathrm{g}-\mathrm{C}_{3} \mathrm{~N}_{4}$ & g- $\mathrm{C}_{3} \mathrm{~N}_{4} /$ Thermal exfoliation & Chemical precipitation & [64] \\
\hline g- $\mathrm{C}_{3} \mathrm{~N}_{4} / \mathrm{Bi}_{12} \mathrm{O}_{17} \mathrm{C}_{12}$ & g- $\mathrm{C}_{3} \mathrm{~N}_{4} /$ Thermal exfoliation & Chemical precipitation & {$[65]$} \\
\hline $\mathrm{MnIn}_{2} \mathrm{~S}_{4} / \mathrm{g}-\mathrm{C}_{3} \mathrm{~N}_{4}$ & g- $\mathrm{C}_{3} \mathrm{~N}_{4} /$ Frozen expansion and post-thermal exfoliation & Hydrothermal & {$[66]$} \\
\hline $\mathrm{CuInS}_{4} / \mathrm{ZnIn}_{2} \mathrm{~S}_{4}$ & $\mathrm{ZnIn}_{2} \mathrm{~S}_{4} /$ Hydrothermal & Hydrothermal & [67] \\
\hline $\mathrm{CuInS}_{4} / \mathrm{g}-\mathrm{C}_{3} \mathrm{~N}_{4}$ & g- $\mathrm{C}_{3} \mathrm{~N}_{4} /$ Thermal exfoliation & Hydrothermal & [68] \\
\hline $\mathrm{C}_{3} \mathrm{~N}_{4} / \mathrm{SnS}_{2}$ & g- $\mathrm{C}_{3} \mathrm{~N}_{4} /$ Ultrasonic exfoliation & Hydrothermal & [69] \\
\hline $\mathrm{MoS}_{2} / \mathrm{SnNb}_{2} \mathrm{O}_{6}$ & $\mathrm{SnNb}_{2} \mathrm{O}_{6} /$ Hydrothermal & Hydrothermal & [70] \\
\hline $\mathrm{SnNb}_{2} \mathrm{O}_{6} / \mathrm{Bi}_{2} \mathrm{WO}_{6}$ & $\mathrm{SnNb}_{2} \mathrm{O}_{6} /$ Hydrothermal & Hydrothermal & [71] \\
\hline $\mathrm{ZnIn}_{2} \mathrm{~S}_{4} / \mathrm{BiOCl}$ & $\mathrm{ZnIn}_{2} \mathrm{~S}_{4} /$ Hydrothermal & Hydrothermal & [72] \\
\hline $\mathrm{P}-\mathrm{La}_{2} \mathrm{Ti}_{2} \mathrm{O}_{7} / \mathrm{Bi}_{2} \mathrm{WO}_{6}$ & $\mathrm{P}-\mathrm{La}_{2} \mathrm{Ti}_{2} \mathrm{O}_{7} /$ Hydrothermal + Calcination & Solvothermal & [73] \\
\hline $\mathrm{MoS}_{2} / \mathrm{PbS}$ & $\mathrm{MoS}_{2} /$ Liquid-phase exfoliation & Solvothermal & [74] \\
\hline $\mathrm{Ti}_{3} \mathrm{C}_{2} @ \mathrm{TiO}_{2} @ \mathrm{MoS}_{2}$ & $\mathrm{Ti}_{3} \mathrm{C}_{2} /$ HF Etching $\mathrm{Ti}_{3} \mathrm{C}_{2} / \mathrm{TiO}_{2} /$ Hydrothermal & Hydrothermal & [75] \\
\hline $\mathrm{BiOCl} / \mathrm{K}^{+} \mathrm{Ca}_{2} \mathrm{Nb}_{3} \mathrm{O}_{10}{ }^{-}$ & $\mathrm{K}^{+} \mathrm{Ca}_{2} \mathrm{Nb}_{3} \mathrm{O}_{10}{ }^{-} /$Solid-phase reaction & Hydrothermal & [76] \\
\hline $\mathrm{CdIn}_{2} \mathrm{~S}_{4} / \mathrm{N}-\mathrm{rGO}$ & N-rGO/Ultrasonic treatment & Hydrothermal & [77] \\
\hline $\mathrm{SnS}_{2} / \mathrm{TiO}_{2}$ & $\mathrm{TiO}_{2} /$ Hydrothermal & Hydrothermal & [78] \\
\hline $\mathrm{WS}_{2} / \mathrm{TiO}_{2}$ & $\mathrm{TiO}_{2} /$ Hydrothermal & Hydrothermal & [79] \\
\hline $\mathrm{GO} / \mathrm{Bi}_{2} \mathrm{WO}_{6}$ & GO/Hummer's method and reduction & Hydrothermal & {$[80]$} \\
\hline $\mathrm{MoS}_{2} / \mathrm{g}-\mathrm{C}_{3} \mathrm{~N}_{4}$ & g- $\mathrm{C}_{3} \mathrm{~N}_{4} /$ Liquid exfoliation & Solvothermal & [81] \\
\hline Black phosphorus (BP)/ $\mathrm{MoS}_{2}$ & BP/Ultrasonic exfoliation & Solvothermal & [82] \\
\hline $\mathrm{SnS}_{2} / \mathrm{MoS}_{2}$ & $\mathrm{SnS}_{2} /$ Hydrothermal & Hydrothermal & {$[83]$} \\
\hline $\mathrm{Zn}_{x} \mathrm{Cd}_{1-x} \mathrm{In}_{2} \mathrm{~S}_{4} / \mathrm{g}-\mathrm{C}_{3} \mathrm{~N}_{4}$ & g- $\mathrm{C}_{3} \mathrm{~N}_{4} /$ Thermal exfoliation & Hydrothermal & [84] \\
\hline $\mathrm{CdS} / \mathrm{g}-\mathrm{C}_{3} \mathrm{~N}_{4}$ & g- $\mathrm{C}_{3} \mathrm{~N}_{4} /$ Liquid exfoliation & Hydrothermal & [85] \\
\hline
\end{tabular}



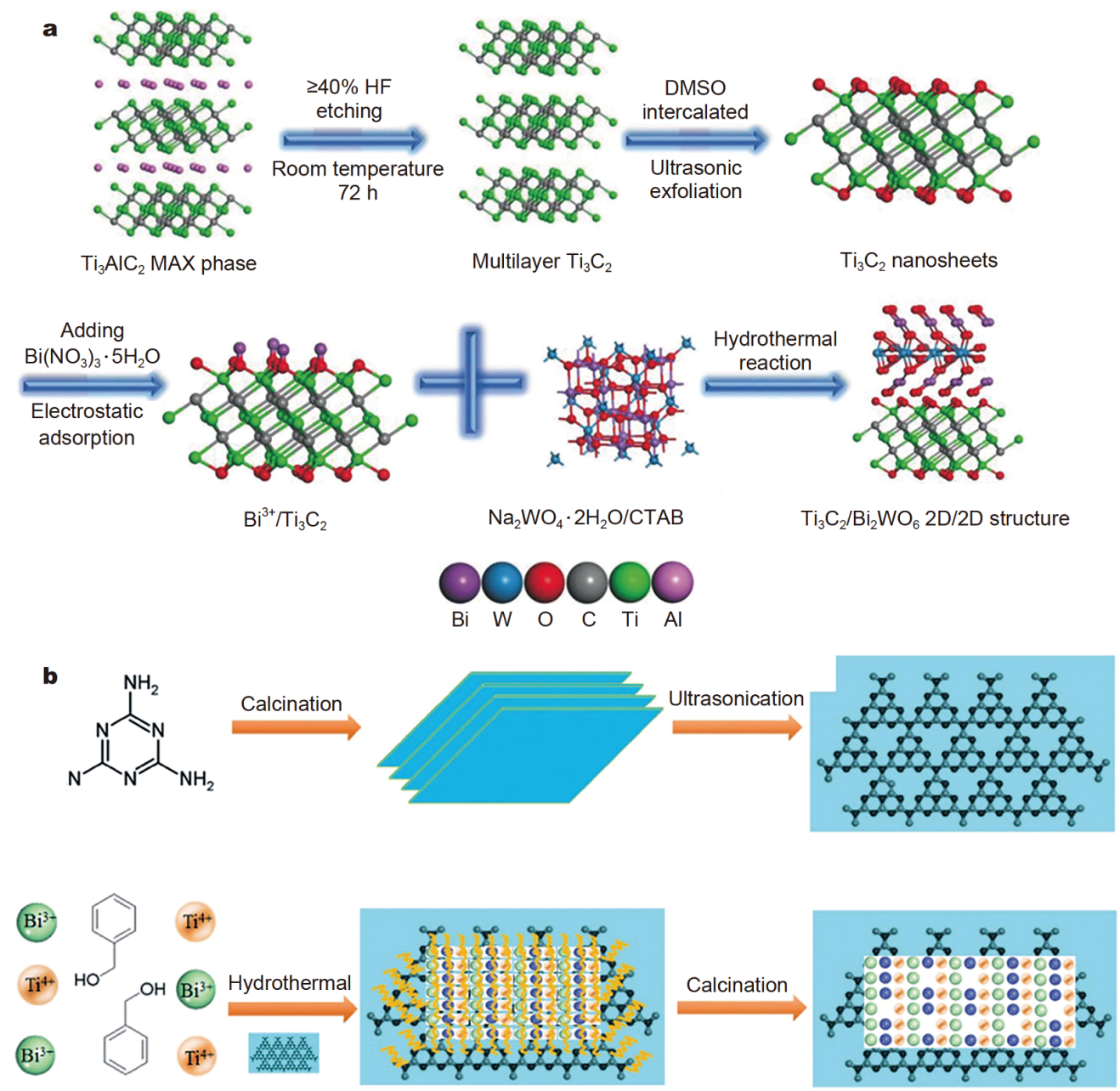

Figure 4 (a) Schematic illustration of the synthetic process for $2 \mathrm{D} / 2 \mathrm{D} \mathrm{Ti}_{3} \mathrm{C}_{2} / \mathrm{Bi}_{2} \mathrm{WO}_{6}$ heterojunction. DMSO: dimethyl sulfoxide. Reprinted with permission from Ref. [28]. Copyright 2018, Wiley-VCH. (b) Schematic illustration of the synthetic process for $2 \mathrm{D} / 2 \mathrm{D} \mathrm{C} \mathrm{C}_{3} / \mathrm{Bi}_{20} \mathrm{TiO}_{32}$ heterojunction. Reprinted with permission from Ref. [29]. Copyright 2015, Royal Society of Chemistry.

nosheets by addition of tetramethylammonium hydroxide (TMA.OH) and $\mathrm{H}_{2} \mathrm{O}_{2}$ in the suspension, and thus the 2D/ $2 \mathrm{D}$ g- $\mathrm{C}_{3} \mathrm{~N}_{4} / \mathrm{MnO}_{2}$ heterostructured photocatalyst was synthesized (Fig. 5b). In another study, Qian and coworkers [31] successfully constructed $\mathrm{Bi}_{4} \mathrm{Ti}_{3} \mathrm{O}_{12} / \mathrm{I}-\mathrm{BiOCl}$ 2D/2D heterojunction systems via an in situ ion exchange approach at room temperature. As displayed in Fig. 5c, the pre-prepared $\mathrm{Bi}_{4} \mathrm{Ti}_{3} \mathrm{O}_{12}$ nanosheets served as the substrate. By mixing with $\mathrm{HCl}$, the outside of the $\mathrm{Bi}_{4} \mathrm{Ti}_{3}$ $\mathrm{O}_{12}$ nanosheets could be dissolved to $\mathrm{Bi}^{3+}$ and $\mathrm{Ti}^{4+}$, and then an intermediate product $\mathrm{BiO}^{+}$was generated because of the hydrolysis of $\mathrm{Bi}^{3+}$. Finally, the second phase I$\mathrm{BiOCl}$ on the surface of the $\mathrm{Bi}_{4} \mathrm{Ti}_{3} \mathrm{O}_{12}$ nanosheets was gradually formed via the combination of $\mathrm{BiO}^{+}, \mathrm{Cl}^{-}$, and $\mathrm{I}^{-}$, and thus the $\mathrm{Bi}_{4} \mathrm{Ti}_{3} \mathrm{O}_{12} / \mathrm{I}-\mathrm{BiOCl} 2 \mathrm{D} / 2 \mathrm{D}$ heterojunction was synthesized (Fig. 5d)
Besides the wet-chemical method, CVD technique was also employed for in-situ growth of the second 2D component on the primarily synthesized $2 \mathrm{D}$ component. Typically, the pre-synthesized $2 \mathrm{D}$ substrate exposed to volatile precursors, which would react or decompose on the surface of substrate for growth of the second 2D component. Gong et al. [32] used the CVD method for growing $\mathrm{WSe}_{2}$ on the edge and top surface of pre-synthesized $\mathrm{MoSe}_{2}$, resulting in the formation of $\mathrm{WSe}_{2} /$ $\mathrm{MoSe}_{2}$ 2D/2D heterostructures. In another example, Han et al. [33] reported the in-situ fabrication of ultrathin $\mathrm{SiC}$ nanosheets on the 2D surface of reduced graphene oxide (rGO) through a facial CVD technique. The growth mechanism of $\mathrm{SiC}$ nanosheets was attributed to a vaporsolid reaction between $\mathrm{Si}$ atoms and solid carbon template. 


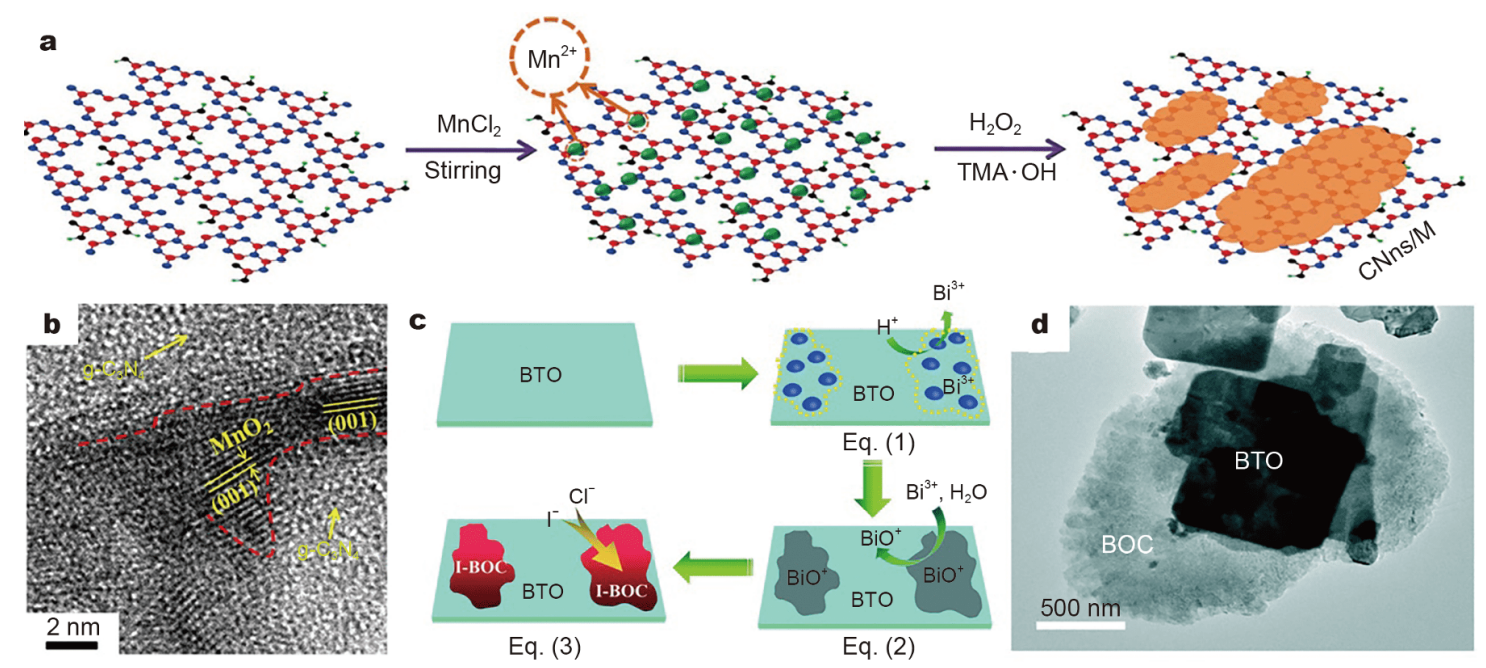

Figure 5 (a, b) Schematic illustration of the synthetic process (a) and representative HRTEM image (b) of $2 \mathrm{D} / 2 \mathrm{D}$ g- $\mathrm{C}_{3} \mathrm{~N}_{4} / \mathrm{MnO}_{2}$ heterojunction. Reprinted with permission from Ref. [30]. Copyright 2017, American Chemical Society. (c, d) Schematic illustration of the synthetic process (c) and representative TEM image (d) of 2D/2D $\mathrm{Bi}_{4} \mathrm{Ti}_{3} \mathrm{O}_{12} / \mathrm{I}-\mathrm{BiOCl}$ heterojunction. Reprinted with permission from Ref. [31]. Copyright 2017, Royal Society of Chemistry.

The 2D/2D heterostructures can also be obtained by a direct synthesis method without the need of pre-synthesized $2 \mathrm{D}$ substrate. For example, Zhang et al. [34] developed a modified nanoconfinement method for the direct synthesis of atomic-scale $2 \mathrm{D} / 2 \mathrm{D}$ heterojunctions of boron nitride and monolayer graphene. In a typical procedure, the $2 \mathrm{D} / 2 \mathrm{D}$ heterojunctions were directly synthesized by pyrolyzing the mixture of glucose (carbon source), boric acid (boron source), and urea (leaving reagent) at the elevated temperature of $1000^{\circ} \mathrm{C}$ under nitrogen atmosphere. More recently, Shi and coworkers [35] designed $\mathrm{Cu}_{2} \mathrm{~S} / \mathrm{Zn}_{0.67} \mathrm{Cd}_{0.33} \mathrm{~S} 2 \mathrm{D} / 2 \mathrm{D}$ nanosheet heterojunctions through a one-step in situ topotactic hydrothermal transformation of CuZnCdAl layered double hydroxide (LDH) precursors.

\section{Ex situ assembly of 2D/2D heterojunction photocatalysts} Compared with the aforementioned in situ growth method, each component in $2 \mathrm{D} / 2 \mathrm{D}$ heterojunction is separately synthesized in this strategy. Then, these $2 \mathrm{D}$ pre-synthesized components are combined together through some assembly strategies, mainly including liquid-phase ultrasonic adsorption, solid phase grinding, electrostatic attraction, hydrothermal and calcination, which are summarized in Table 2 [86-125].

Liquid-phase ultrasonic adsorption is one of the simplest routes, which is often carried by a direct ultrasonic treatment of the mixed suspension of the $2 \mathrm{D}$ components. Ma et al. [86] constructed 2D CdS/MoS 2 heterojunction by ultrasonic adsorption of CdS nanosheets and
$\mathrm{MoS}_{2}$ nanosheets. As shown in Fig. 6a, the CdS nanosheets and $\mathrm{MoS}_{2}$ nanosheets were firstly synthesized by the solvothermal and hydrothermal method, respectively. Then, a certain amount of the CdS nanosheets as well as $\mathrm{MoS}_{2}$ nanosheets were dispersed into water to form a mixed suspension. The CdS/MoS 2 composites were obtained after the ultrasonic treatment of the mixed suspension and followed vacuum-drying. In another study, Qiao's group [87] fabricated a metal-free phosphorene (FP)/graphitic carbon nitride (CNS) 2D/2D heterojunction through mechanically grinding the FP/CNS ethanol suspension. The TEM image (Fig. 6b) clearly revealed that the 2D CNS was attached on the surface of ultrathin FP based on weak vdW force. Solid phase grinding along with post-sintering process was also employed to assemble the 2D/2D heterojunction. Xu and coworkers [88] successfully synthesized a $\mathrm{Bi}_{4} \mathrm{NbO}_{8} \mathrm{Cl} / \mathrm{g}-\mathrm{C}_{3} \mathrm{~N}_{4} \quad 2 \mathrm{D} / 2 \mathrm{D}$ heterojunction via the solid phase grinding and postsintering process. As shown in Fig. $6 \mathrm{c}, \mathrm{Bi}_{4} \mathrm{NbO}_{8} \mathrm{Cl}$ and g$\mathrm{C}_{3} \mathrm{~N}_{4}$ nanosheets were firstly prepared by a molten-salt and thermal polymerization method, respectively. Then, the $\mathrm{Bi}_{4} \mathrm{NbO}_{8} \mathrm{Cl}$ and $\mathrm{g}-\mathrm{C}_{3} \mathrm{~N}_{4}$ nanosheets mixed together by ball-milling as well as further calcination in air. Similarly, Liu et al. [89] also used this strategy to fabricate 2D/2D $\mathrm{Bi}_{4} \mathrm{Ti}_{3} \mathrm{O}_{12} / \mathrm{Ni}(\mathrm{OH})_{2}$ composites.

Although the coupling of $2 \mathrm{D}$ components via liquidphase ultrasonic adsorption or solid phase grinding is a simple and direct route, the interface adhesion in such obtained $2 \mathrm{D} / 2 \mathrm{D}$ systems is usually not strong, which may result in the breaking down of their structures in practical 
Table 2 Summary of the ex situ methods to assembly of 2D/2D heterojunction

\begin{tabular}{|c|c|c|c|c|}
\hline 2D/2D heterojunction & Component A/Method & Component B/Method & Assembly methods & References \\
\hline $\mathrm{SnNb}_{2} \mathrm{O}_{6} /$ Graphene & $\begin{array}{l}\mathrm{SnNb}_{2} \mathrm{O}_{6} /(\mathrm{Hydrothermal}+\text { Calcination }+ \\
\text { Positively-charged functionalization })\end{array}$ & $\begin{array}{l}\text { Graphene/(Modified Hummers } \\
\text { method + BPEI refluxing) }\end{array}$ & Electrostatic attraction & {$[95]$} \\
\hline $\mathrm{rGO} / \mathrm{g}-\mathrm{C}_{3} \mathrm{~N}_{4}$ & $\begin{array}{l}\text { g- } \mathrm{C}_{3} \mathrm{~N}_{4} /(\text { Thermal polymerization + Ultra- } \\
\text { sonic exfoliation + Proton-functionalized) }\end{array}$ & $\begin{array}{c}\mathrm{rGO} / \text { (Modified Hummers method }+ \\
\text { Ultrasonic exfoliation }+\mathrm{NaBH}_{4} \text { re- } \\
\text { duction) }\end{array}$ & Electrostatic attraction & {$[96]$} \\
\hline $\mathrm{SnS}_{2} / g-\mathrm{C}_{3} \mathrm{~N}_{4}$ & $\mathrm{SnS}_{2} /$ Hydrothermal & $\begin{array}{c}\mathrm{g}-\mathrm{C}_{3} \mathrm{~N}_{4} /(\text { Thermal polymerization }+ \\
\text { Ultrasonic exfoliation) }\end{array}$ & $\begin{array}{l}\text { Ultrasonic adsorption } \\
+ \text { Hydrothermal }\end{array}$ & [97] \\
\hline $\mathrm{C}_{3} \mathrm{~N}_{4} / \mathrm{rGO}$ & $\begin{array}{c}\mathrm{C}_{3} \mathrm{~N}_{4} /(\text { Thermal polymerization + Ultrasonic } \\
\text { exfoliation + Proton-functionalized })\end{array}$ & rGO/Modified Hummers method & $\begin{array}{l}\text { Photo-assisted electro- } \\
\text { static attraction }\end{array}$ & [98] \\
\hline $\mathrm{Bi}_{2} \mathrm{O}_{2} \mathrm{CO}_{3} / g-\mathrm{C}_{3} \mathrm{~N}_{4}$ & $\mathrm{Bi}_{2} \mathrm{O}_{2} \mathrm{CO}_{3} /$ Hydrothermal & g- $\mathrm{C}_{3} \mathrm{~N}_{4} /$ Thermal polymerization & Calcination & {$[99]$} \\
\hline $\mathrm{MoS}_{2} / \mathrm{g}-\mathrm{C}_{3} \mathrm{~N}_{4}$ & $\begin{array}{c}\mathrm{MoS}_{2} / \text { Hydrothermal + Ultrasonic } \\
\text { exfoliation }\end{array}$ & $\begin{array}{c}\mathrm{g}-\mathrm{C}_{3} \mathrm{~N}_{4} /(\text { Thermal polymerization }+ \\
\text { Ultrasonic exfoliation) }\end{array}$ & $\begin{array}{l}\text { Impregnation and } \\
\text { calcination method }\end{array}$ & {$[100]$} \\
\hline $\mathrm{GL}-\mathrm{MoS}_{2} / \mathrm{C}_{3} \mathrm{~N}_{4}$ & $\mathrm{MoS}_{2} /$ Hydrothermal & $\mathrm{C}_{3} \mathrm{~N}_{4} /$ Thermal polymerization & Hydrothermal & {$[101]$} \\
\hline $\mathrm{SnNb}_{2} \mathrm{O}_{6} / g-\mathrm{C}_{3} \mathrm{~N}_{4}$ & $\mathrm{SnNb}_{2} \mathrm{O}_{6} /$ Hydrothermal & $\begin{array}{c}\mathrm{g}-\mathrm{C}_{3} \mathrm{~N}_{4} /(\text { Thermal polymerization }+ \\
\left.\mathrm{HNO}_{3} \text { exfoliation }\right)\end{array}$ & Hydrothermal & {$[102]$} \\
\hline $\mathrm{C}_{3} \mathrm{~N}_{4} / \mathrm{GO}$ & $\mathrm{C}_{3} \mathrm{~N}_{4} /$ Thermal polymerization & GO/Modified Hummers method & $\begin{array}{l}\text { Ultrasonic adsorption } \\
\quad+\text { Freeze drying }\end{array}$ & {$[103]$} \\
\hline $\mathrm{GO} / \mathrm{g}-\mathrm{C}_{3} \mathrm{~N}_{4}$ & GO/Modified Hummers method & $\begin{array}{c}\mathrm{g}-\mathrm{C}_{3} \mathrm{~N}_{4} /(\text { Thermal polymerization }+ \\
\text { Ultrasonic exfoliation + Proton- } \\
\text { functionalized })\end{array}$ & $\begin{array}{l}\text { Photo-assisted electro- } \\
\text { static attraction }\end{array}$ & {$[104]$} \\
\hline $\mathrm{Bi}_{2} \mathrm{WO}_{6} / \mathrm{TiO}_{2}$ & $\begin{array}{c}\mathrm{TiO}_{2} /(\text { Hydrothermal }+ \text { Positively-charged } \\
\text { functionalization })\end{array}$ & $\begin{array}{l}\mathrm{Bi}_{2} \mathrm{WO}_{6} /(\text { Hydrothermal }+ \\
\text { A-TNS functionalization) }\end{array}$ & Electrostatic attraction & {$[91]$} \\
\hline $\mathrm{g}-\mathrm{C}_{3} \mathrm{~N}_{4} / \mathrm{K}^{+} \mathrm{Ca}_{2} \mathrm{Nb}_{3} \mathrm{O}_{10}^{-}$ & $\begin{array}{c}\mathrm{K}^{+} \mathrm{Ca}_{2} \mathrm{Nb}_{3} \mathrm{O}_{10}^{-} / \mathrm{TBA}^{+} \mathrm{OH}^{-} \text {ultrasonic } \\
\text { exfoliation }\end{array}$ & g- $\mathrm{C}_{3} \mathrm{~N}_{4} /$ Thermal polymerization & Hydrothermal & {$[93]$} \\
\hline $\mathrm{WO}_{3} / \mathrm{K}^{+} \mathrm{Ca}_{2} \mathrm{Nb}_{3} \mathrm{O}_{10}^{-}$ & $\mathrm{WO}_{3} /$ Hydrothermal & $\begin{array}{c}\mathrm{K}^{+} \mathrm{Ca}_{2} \mathrm{Nb}_{3} \mathrm{O}_{10}^{-} / \mathrm{TBAOH}^{-} \text {ultrasonic } \\
\text { exfoliation }\end{array}$ & Hydrothermal & {$[94]$} \\
\hline N-doped $\mathrm{La}_{2} \mathrm{Ti}_{2} \mathrm{O}_{7} / \mathrm{g}-\mathrm{C}_{3} \mathrm{~N}_{4}$ & $\mathrm{~N}$-doped $\mathrm{La}_{2} \mathrm{Ti}_{2} \mathrm{O}_{7} /$ Hydrothermal & $\begin{array}{c}\mathrm{g}-\mathrm{C}_{3} \mathrm{~N}_{4} /(\text { Thermal polymerization }+ \\
\text { Thermal exfoliation) }\end{array}$ & Ultrasonic adsorption & {$[105]$} \\
\hline $\mathrm{CdS} / \mathrm{MoO}_{2}$ & CdS/Solvothermal & $\mathrm{MoO}_{2} /$ Hydrothermal & Ultrasonic adsorption & {$[86]$} \\
\hline $\mathrm{WO}_{3} / \mathrm{SnNb}_{2} \mathrm{O}_{6}$ & $\mathrm{WO}_{3} /$ Hydrothermal & $\mathrm{SnNb}_{2} \mathrm{O}_{6} /$ Hydrothermal & Hydrothermal & {$[106]$} \\
\hline $\mathrm{BiOI} / \mathrm{CeO}_{2}$ & $\mathrm{CeO}_{2} /$ Refluxed method & $\mathrm{BiOI} /$ Precipitation & Ultrasonic adsorption & {$[107]$} \\
\hline $\mathrm{BP} / \mathrm{g}-\mathrm{C}_{3} \mathrm{~N}_{4}$ & BP/NMP solvent exfoliation & $\begin{array}{c}\mathrm{g}-\mathrm{C}_{3} \mathrm{~N}_{4} /(\text { Thermal polymerization }+ \\
\text { Thermal exfoliation) }\end{array}$ & Ultrasonic adsorption & {$[108]$} \\
\hline $\mathrm{BiOIO}_{3} / \mathrm{g}-\mathrm{C}_{3} \mathrm{~N}_{4}$ & $\begin{array}{c}\mathrm{g}-\mathrm{C}_{3} \mathrm{~N}_{4} /(\text { Thermal polymerization }+ \text { Ultra- } \\
\text { sonic exfoliation })\end{array}$ & $\begin{array}{l}\mathrm{BiOIO}_{3} \text { (Hydrothermal + Positively- } \\
\text { charged functionalization) }\end{array}$ & Electrostatic attraction & [109] \\
\hline $\mathrm{rGO} / \mathrm{g}-\mathrm{C}_{3} \mathrm{~N}_{4}$ & $\begin{array}{l}\text { g-C } \mathrm{C}_{3} \mathrm{~N}_{4} /(\text { Thermal polymerization + Ultra- } \\
\text { sonic exfoliation + Proton-functionalized })\end{array}$ & GO/Modified Hummers method & Electrostatic attraction & {$[110]$} \\
\hline Porous-g- $\mathrm{C}_{3} \mathrm{~N}_{4} / \mathrm{Bi}_{2} \mathrm{WO}_{6}$ & Porous-g- $\mathrm{C}_{3} \mathrm{~N}_{4} /$ Thermal polymerization & $\mathrm{Bi}_{2} \mathrm{WO}_{6} /$ Hydrothermal & Ultrasonic adsorption & {$[111]$} \\
\hline $\mathrm{Ni}_{2} \mathrm{P} / \mathrm{ZnIn}_{2} \mathrm{~S}_{4}$ & $\mathrm{ZnIn}_{2} \mathrm{~S}_{4} /$ Hydrothermal + Ultrasonic exfo- & $\mathrm{Ni}_{2} \mathrm{P} / \mathrm{Hydrothermal}+$ Calcination & Ultrasonic adsorption & [112] \\
\hline $\mathrm{CdS} / \mathrm{WS}_{2}$ & CdS/Hydrothermal & $\mathrm{WS}_{2} / \mathrm{NMP}$ ultrasonic exfoliation & Stirring adsorption & {$[113]$} \\
\hline $\mathrm{MoO}_{2} / \mathrm{GL}-\mathrm{C}_{3} \mathrm{~N}_{4}$ & $\begin{array}{c}\mathrm{MoO}_{2} / \text { Interfacial self-assembly and thermal } \\
\text { reduction }\end{array}$ & $\begin{array}{c}\mathrm{g}-\mathrm{C}_{3} \mathrm{~N}_{4} /(\text { Thermal polymerization }+ \\
\text { Thermal exfoliation })\end{array}$ & Hydrothermal & [114] \\
\hline Phosphorene/g- $\mathrm{C}_{3} \mathrm{~N}_{4}$ & Phosphorene/Ultrasonic exfoliation & $\begin{array}{c}\mathrm{g}-\mathrm{C}_{3} \mathrm{~N}_{4} /(\text { Thermal polymerization }+ \\
\text { Thermal exfoliation })\end{array}$ & Mechanically ground & {$[87]$} \\
\hline $\mathrm{Fe}_{2} \mathrm{O}_{3} / g-\mathrm{C}_{3} \mathrm{~N}_{4}$ & $\mathrm{Fe}_{2} \mathrm{O}_{3} /$ Hydrothermal & $\begin{array}{c}\text { g- } \mathrm{C}_{3} \mathrm{~N}_{4} /(\text { Thermal polymerization }+ \\
\text { Ultrasonic exfoliation + Proton- } \\
\text { functionalized) }\end{array}$ & Electrostatic attraction & {$[92]$} \\
\hline $\mathrm{g}-\mathrm{C}_{3} \mathrm{~N}_{4} / \mathrm{MoS}_{2}$ & $\begin{array}{c}\mathrm{g}-\mathrm{C}_{3} \mathrm{~N}_{4} /(\text { Thermal polymerization }+ \text { Thermal } \\
\text { exfoliation })\end{array}$ & $\mathrm{MoS}_{2} /$ Ultrasonic exfoliation & Ultrasonic adsorption & [115] \\
\hline $\mathrm{WO}_{3} / \mathrm{g}-\mathrm{C}_{3} \mathrm{~N}_{4}$ & $\begin{array}{l}\text { g- } \mathrm{C}_{3} \mathrm{~N}_{4} /(\text { Thermal polymerization + Ultra- } \\
\text { sonic exfoliation + Positively-charged } \\
\text { functionalization) }\end{array}$ & $\begin{array}{l}\mathrm{WO}_{3} / \mathrm{BSA} \text { electrostatic-assisted } \\
\text { ultrasonic exfoliation }\end{array}$ & Electrostatic attraction & {$[116]$} \\
\hline
\end{tabular}


(Continued)

\begin{tabular}{|c|c|c|c|c|}
\hline 2D/2D heterojunction & Component A/Method & Component B/Method & Assembly methods & References \\
\hline $\mathrm{ZnIn}_{2} \mathrm{~S}_{4} / \mathrm{MoS}_{2}$ & $\mathrm{ZnIn}_{2} \mathrm{~S}_{4} /($ Hydrothermal + Cryodesiccation) & $\begin{array}{l}\mathrm{MoS}_{2} /(\text { Hydrothermal }+ \\
\quad \text { Cryodesiccation })\end{array}$ & Electrostatic attraction & {$[117]$} \\
\hline $\begin{array}{c}\mathrm{Ti}_{3} \mathrm{C}_{2} \underset{\mathrm{g}-\mathrm{C}_{3} \mathrm{~N}_{4}}{\mathrm{MXene} / \mathrm{O} \text {-doped }} \\
.\end{array}$ & $\begin{array}{c}\mathrm{g}-\mathrm{C}_{3} \mathrm{~N}_{4} /(\text { Thermal polymerization }+ \\
\text { Ultrasonic exfoliation + Calcination }+ \\
\text { Proton-functionalized })\end{array}$ & $\begin{array}{c}\mathrm{Ti}_{3} \mathrm{C}_{2} /(\mathrm{HF} \text { etching }+ \text { Ultrasonic } \\
\text { exfoliation })\end{array}$ & Electrostatic attraction & {$[118]$} \\
\hline $\mathrm{Ti}_{3} \mathrm{C}_{2} / g-\mathrm{C}_{3} \mathrm{~N}_{4}$ & $\mathrm{Ti}_{3} \mathrm{C}_{2} /$ Pyroreaction $+\mathrm{HF}$ etching & $\begin{array}{c}\text { g- } \mathrm{C}_{3} \mathrm{~N}_{4} /(\text { Thermal polymerization }+ \\
\text { Thermal exfoliation + Calcination }+ \\
\text { Proton-functionalized })\end{array}$ & Electrostatic attraction & [119] \\
\hline $\mathrm{BiVO}_{4} / \mathrm{g}-\mathrm{C}_{3} \mathrm{~N}_{4}$ & $\mathrm{BiVO}_{4} /$ Hydrothermal & g- $\mathrm{C}_{3} \mathrm{~N}_{4} /$ Thermal polymerization & Ultrasonic adsorption & {$[120]$} \\
\hline $\mathrm{WO}_{3} / \mathrm{ZnIn}_{2} \mathrm{~S}_{4}$ & $\begin{array}{l}\mathrm{WO}_{3} /(\text { Hydrothermal + Calcination }+ \\
\text { Positively-charged functionalization })\end{array}$ & $\underset{\text { exfoliation })}{\mathrm{ZnIn}_{2} \mathrm{~S}_{4} /(\text { Fefluxing }+ \text { Ultrasonic }}$ & Electrostatic attraction & {$[90]$} \\
\hline $\mathrm{ZnIn}_{2} \mathrm{~S}_{4} / \mathrm{g}-\mathrm{C}_{3} \mathrm{~N}_{4}$ & $\begin{array}{c}\text { g- } \mathrm{C}_{3} \mathrm{~N}_{4} /(\text { Thermal polymerization }+ \\
\text { Ultrasonic exfoliation + Calcination }+ \\
\text { Proton-functionalized })\end{array}$ & $\begin{array}{c}\mathrm{ZnIn}_{2} \mathrm{~S}_{4} /(\text { Fefluxing }+ \text { Ultrasonic } \\
\text { exfoliation })\end{array}$ & Electrostatic attraction & {$[121]$} \\
\hline $\mathrm{ZnV}_{2} \mathrm{O}_{6} / g-\mathrm{C}_{3} \mathrm{~N}_{4}$ & $\mathrm{ZnV}_{2} \mathrm{O}_{6} /($ Hydrothermal + Calcination $)$ & $\begin{array}{c}\mathrm{g}-\mathrm{C}_{3} \mathrm{~N}_{4} /(\text { Thermal polymerization }+ \\
\text { Proton-functionalized })\end{array}$ & Electrostatic attraction & {$[122]$} \\
\hline $\mathrm{SnS}_{2} / g-\mathrm{C}_{3} \mathrm{~N}_{4}$ & $\begin{array}{c}\mathrm{g}-\mathrm{C}_{3} \mathrm{~N}_{4} /(\text { Thermal polymerization }+ \text { Thermal } \\
\text { exfoliation })\end{array}$ & $\mathrm{SnS}_{2} /$ Hydrothermal & Hydrothermal & {$[123]$} \\
\hline $\mathrm{BP} / \mathrm{g}-\mathrm{C}_{3} \mathrm{~N}_{4}$ & BP/Solvent exfoliation & $\begin{array}{c}\mathrm{g}-\mathrm{C}_{3} \mathrm{~N}_{4} /(\text { Thermal polymerization }+ \\
\text { Thermal exfoliation) }\end{array}$ & Ultrasonic adsorption & {$[124]$} \\
\hline $\mathrm{Bi}_{4} \mathrm{Ti}_{3} \mathrm{O}_{12} / \mathrm{Ni}(\mathrm{OH})_{2}$ & $\mathrm{Bi}_{4} \mathrm{Ti}_{3} \mathrm{O}_{12} /$ Molten salt method & $\mathrm{Ni}(\mathrm{OH})_{2} /$ Sedimentation & Solid phase grinding & {$[89]$} \\
\hline $\mathrm{Bi}_{4} \mathrm{NbO}_{8} \mathrm{Cl} / \mathrm{g}-\mathrm{C}_{3} \mathrm{~N}_{4}$ & $\mathrm{Bi}_{4} \mathrm{NbO}_{8} \mathrm{Cl} / \mathrm{Molten}$ salt method & g- $\mathrm{C}_{3} \mathrm{~N}_{4} /$ Thermal polymerization & $\begin{array}{l}\text { Solid phase grinding }+ \\
\text { Calcination }\end{array}$ & {$[88]$} \\
\hline $\mathrm{Bi}_{2} \mathrm{WO}_{6} / \mathrm{BiOI}$ & $\mathrm{Bi}_{2} \mathrm{WO}_{6} /$ Hydrothermal & $\mathrm{BiOI} /$ Sedimentation & Ultrasonic adsorption & {$[125]$} \\
\hline
\end{tabular}

Abbreviations: A-TNS: anatase $\mathrm{TiO}_{2}$ nanosheet; BPEI: branched polyethylenimine; BSA: bovine serum albumin; GL: graphene-like; NMP: $N$ methyl-2-pyrrolidone.

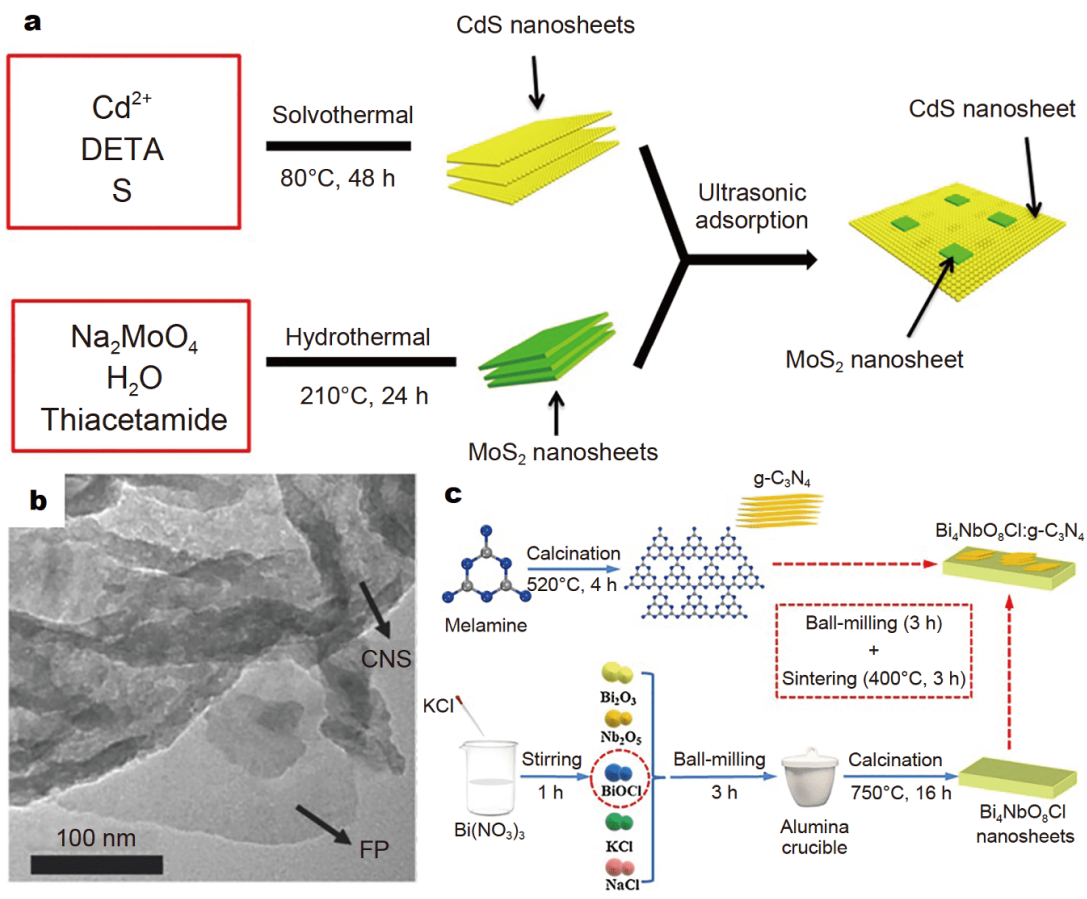

Figure 6 (a) Schematic illustration of the synthetic process of 2D/2D CdS/MoS 2 heterojunction. DETA: diethylenetriamine. Reprinted with permission from Ref. [86]. Copyright 2017, Elsevier. (b) TEM image of metal-FP/graphitic CNS 2D/2D heterojunction. Reprinted with permission from Ref. [87]. Copyright 2018, Wiley-VCH. (c) Schematic illustration of the synthetic process of 2D/2D Bi $\mathrm{NbO}_{8} \mathrm{Cl} / \mathrm{g}-\mathrm{C}_{3} \mathrm{~N}_{4}$ heterojunction. Reprinted with permission from Ref. [88]. Copyright 2019, Elsevier. 
applications. Thus, some approaches have been developed to consolidate their heterojunction interfaces. One of the most effective strategies is to respectively modify each $2 \mathrm{D}$ component with heterogeneous charges. Then, an electrostatic attraction occur between them when they mix together, which benefits the fabrication of the $2 \mathrm{D} / 2 \mathrm{D}$ heterojunction with good interfacial contact. According to this route, a variety of advanced $2 \mathrm{D} / 2 \mathrm{D}$ heterojunctions have been synthesized, which are summarized in Table 2. For example, Tan et al. [90] constructed 2D/2D $\mathrm{WO}_{3} / \mathrm{ZnIn}_{2} \mathrm{~S}_{4}$ nanocomposites via electrostatic attraction between modified $\mathrm{WO}_{3}$ and $\mathrm{ZnIn}_{2} \mathrm{~S}_{4}$. As shown in Fig. 7a, $\mathrm{WO}_{3}$ nanosheets were synthesized through a hydrothermal process and post-annealing treatment while the negatively charged $\mathrm{ZnIn}_{2} \mathrm{~S}_{4}$ nanosheets were obtained by a refluxing and ultrasonic exfoliation process. The $\mathrm{WO}_{3}$ nanosheets were further modified by adding 3-aminopropyltriethoxysilane (APTES) to obtain positivelycharged $\mathrm{WO}_{3}$ disperse solution, and then the negatively charged $\mathrm{ZnIn}_{2} \mathrm{~S}_{4}$ nanosheets were added into the suspension to assemble 2D/2D $\quad \mathrm{WO}_{3} / \mathrm{ZnIn}_{2} \mathrm{~S}_{4}$ heterostructure. TEM (Fig. 7b) and HRTEM (Fig. 7c) images revealed the intimate contact interfaces between $\mathrm{WO}_{3}$ and $\mathrm{ZnIn}_{2} \mathrm{~S}_{4}$ nanosheets. Yuan et al. [91] also employed the electrostatic self-assembly method to design a $2 \mathrm{D} / 2 \mathrm{D}$ heterojunction. The positively charged $\mathrm{TiO}_{2}$ nanosheets and negatively charged $\mathrm{Bi}_{2} \mathrm{WO}_{6}$ were combined together to couple the $2 \mathrm{D} / 2 \mathrm{D} \quad \mathrm{Bi}_{2} \mathrm{WO}_{6} / \mathrm{TiO}_{2}$ heterojunction (Fig. 7d). In another study, Xu and coworkers [92] synthesized 2D/2D $\mathrm{Fe}_{2} \mathrm{O}_{3} / g-\mathrm{C}_{3} \mathrm{~N}_{4}$ composites via the facile electrostatic self-assembly approach. As confirmed by the measured Zeta potential results, the strong negative charge on $\mathrm{g}-\mathrm{C}_{3} \mathrm{~N}_{4}$ could provide a significant driving force to attract positively charged $\mathrm{Fe}_{2} \mathrm{O}_{3}$, resulting in spontaneous assembly of the two components into a $2 \mathrm{D} /$ $2 \mathrm{D}$ heterostructure (Fig. 7e).

Hydrothermal co-assembly is also an effective method to couple the pre-synthesized $2 \mathrm{D}$ components. The formed $2 \mathrm{D} / 2 \mathrm{D}$ heterostructure also possesses strong contact interface. Jiang et al. [93] coupled the perovskite oxide ultrathin nanosheets with $\mathrm{g}-\mathrm{C}_{3} \mathrm{~N}_{4}$ nanosheets by the hydrothermal coassembly method. As displayed in Fig. 8a, ultrathin $\mathrm{K}^{+} \mathrm{Ca}_{2} \mathrm{Nb}_{3} \mathrm{O}_{10}{ }^{-}$nanosheets were obtained by exfoliation of the $\mathrm{KCa}_{2} \mathrm{Nb}_{3} \mathrm{O}_{10}$ bulk materials in tetrabutylammonium hydroxide (TBAOH) aqueous solution and the $\mathrm{g}_{-} \mathrm{C}_{3} \mathrm{~N}_{4}$ nanosheets were prepared by a thermal polymerization strategy. Afterwards, a certain amount of the as-prepared $\mathrm{g}-\mathrm{C}_{3} \mathrm{~N}_{4}$ nanosheets and

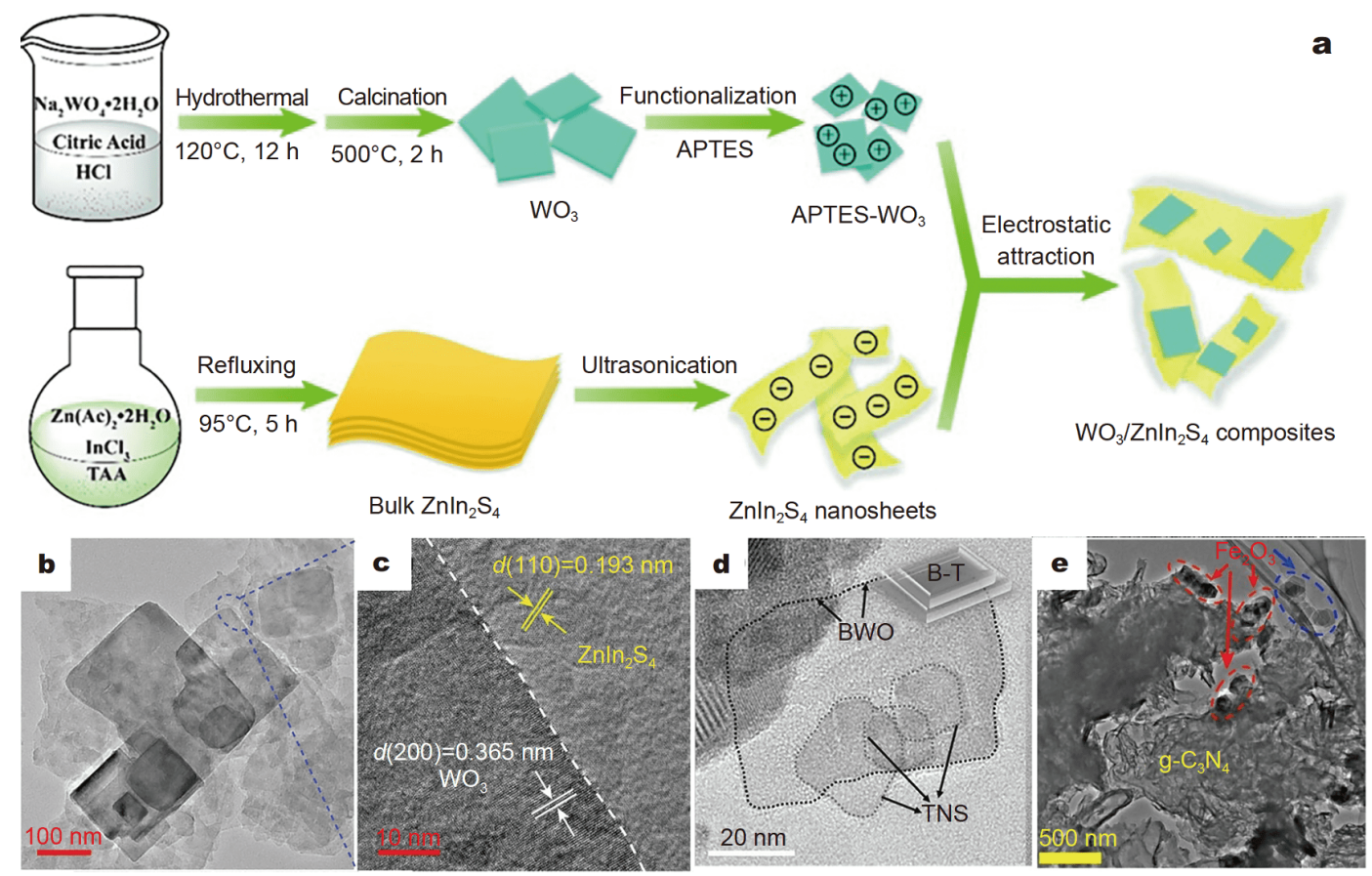

Figure 7 (a-c) Schematic illustration of the synthetic process (a), TEM image (b) and HRTEM image (c) of the $2 \mathrm{D} / 2 \mathrm{D}$ WO $\mathrm{O}_{3} / \mathrm{ZnIn}_{2} \mathrm{~S}_{4}$ heterojunction. Reprinted with permission from Ref. [90]. Copyright 2019, Royal Society of Chemistry. (d) TEM image of the $2 \mathrm{D} / 2 \mathrm{D} \mathrm{Bi}_{2} \mathrm{WO}_{6} / \mathrm{TiO}_{2}$ heterojunction. Reprinted with permission from Ref. [91]. Copyright 2017, Wiley-VCH. (e) TEM image of 2D/2D $\mathrm{Fe}_{2} \mathrm{O}_{3} / \mathrm{g}_{-} \mathrm{C}_{3} \mathrm{~N}_{4}$ composites. Reprinted with permission from Ref. [92]. Copyright 2018, Wiley-VCH. 


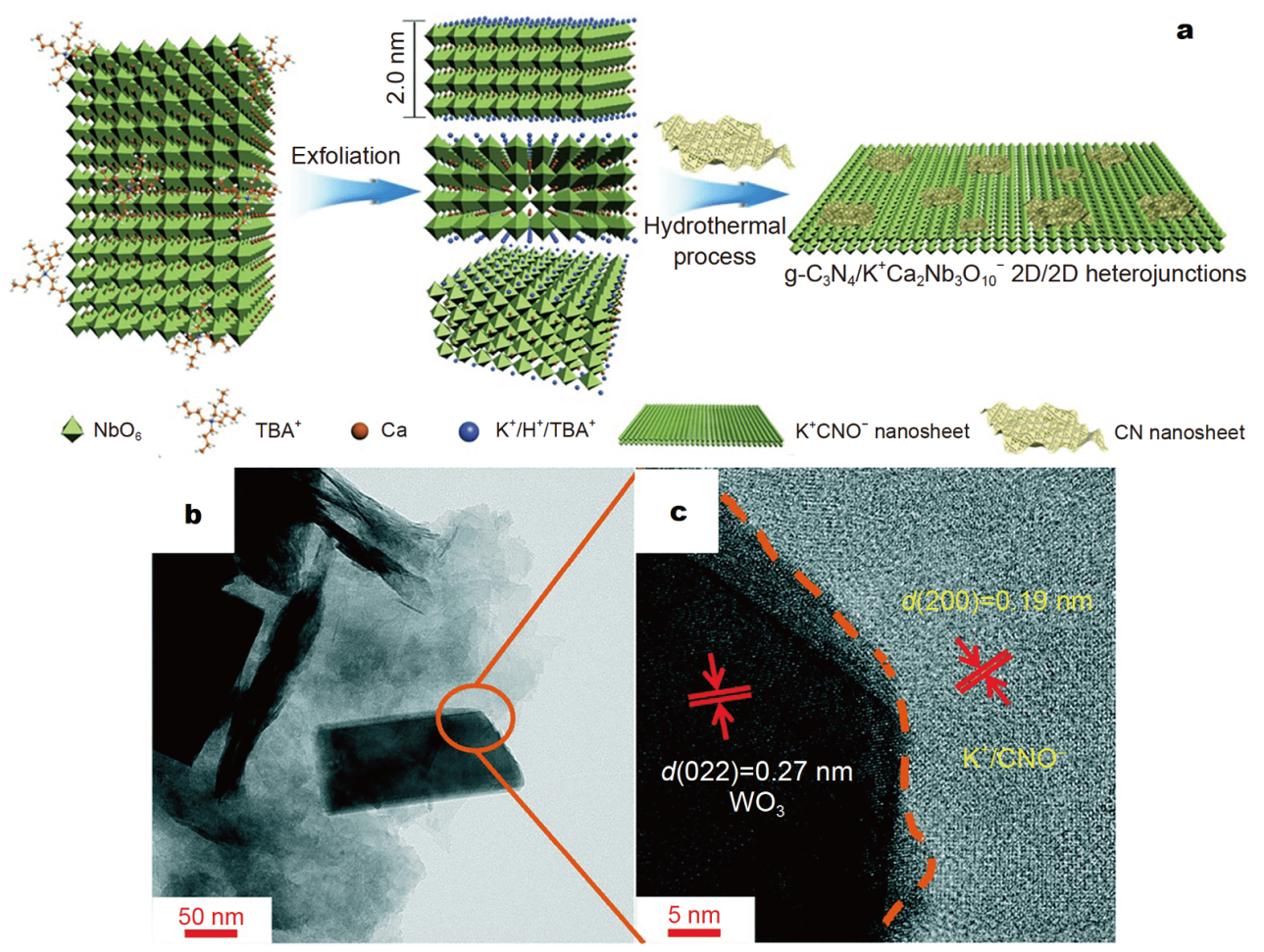

Figure 8 (a) Schematic illustration of the synthetic process of $2 \mathrm{D} / 2 \mathrm{D} \mathrm{g}-\mathrm{C}_{3} \mathrm{~N}_{4} / \mathrm{K}^{+} \mathrm{Ca}_{2} \mathrm{Nb}_{3} \mathrm{O}_{10}{ }^{-}$heterojunction. Reprinted with permission from Ref. [93]. Copyright 2017, Elsevier. (b, c) TEM and HRTEM images of $2 \mathrm{D} / 2 \mathrm{D} \mathrm{WO}_{3} / \mathrm{K}^{+} \mathrm{Ca}_{2} \mathrm{Nb}_{3} \mathrm{O}_{10}$ heterojunction. Reprinted with permission from Ref. [94]. Copyright 2017, Royal Society of Chemistry.

$\mathrm{K}^{+} \mathrm{Ca}_{2} \mathrm{Nb}_{3} \mathrm{O}_{10}{ }^{-}$nanosheets were simultaneously added in deionized water to form a homogeneous suspension. The $2 \mathrm{D} / 2 \mathrm{D}$ g- $\mathrm{C}_{3} \mathrm{~N}_{4} / \mathrm{K}^{+} \mathrm{Ca}_{2} \mathrm{Nb}_{3} \mathrm{O}_{10}{ }^{-}$heterostructure (Fig. 8a) was fabricated by a facile one-step hydrothermal treatment of the mixture suspension. Similarly, Ma and coworkers [94] further coupled the ultrathin $\mathrm{K}^{+} \mathrm{Ca}_{2} \mathrm{Nb}_{3} \mathrm{O}_{10}{ }^{-}$nanosheets with $\mathrm{WO}_{3}$ nanosheets via the hydrothermal coassembly route. The TEM image (Fig. 8b) and HRTEM (Fig. 8c) image confirmed that the $\mathrm{WO}_{3}$ nanosheets and $\mathrm{K}^{+} \mathrm{Ca}_{2} \mathrm{Nb}_{3} \mathrm{O}_{10}{ }^{-}$nanosheets successfully combined each other by forming an intimate $2 \mathrm{D} / 2 \mathrm{D}$ heterojunction interface.

\section{APPLICATIONS OF 2D/2D HETEROJUNCTION PHOTOCATALYSTS}

The 2D/2D heterojunction materials can display huge advantages toward photocatalytic reactions including the preferable dimensionality design, appropriate band structure, and surface properties. To date, the advanced $2 \mathrm{D} / 2 \mathrm{D}$ heterojunction photocatalysts have been widely applied in diverse photocatalytic applications. In this section, the advancement of versatile photocatalytic applications of $2 \mathrm{D} / 2 \mathrm{D}$ heterojunction photocatalysts in the fields of hydrogen generation, environmental purification and $\mathrm{CO}_{2}$ reduction will be discussed (Table 3).

\section{Hydrogen generation}

Hydrogen fuel has been considered as a clean and sustainable form of energy with the advantages including high energy density $\left(142 \mathrm{MJ} \mathrm{kg}^{-1}\right)$, high stability and clean combustion product $[126,127]$. At present, the process of hydrogen generation mainly involves chemical decomposition of biomass [128], electrolysis of water [129], and photocatalytic decomposition of water [130132]. Among these, hydrogen generation via photocatalytic water splitting is a promising eco-friendly route to address the energy crisis [133]. In this technology, it only needs the sustainable solar light as energy input, photocatalysts as medium, and water as reaction source, while there is no pollutive emission in the whole process. Thus, photocatalytic water splitting into hydrogen has been recognized as the "Holy Grail" of the renewable energy research. Up to now, various impressive photocatalyst materials have been developed for efficient and stable photocatalytic hydrogen production [134-139]. However, most of the employed photocatalysts are still 
Table 3 Summary of the photocatalytic performances by using 2D/2D heterojunction photocatalysts

\begin{tabular}{|c|c|c|c|c|c|c|}
\hline Photocatalysts & $\begin{array}{c}\text { Amount of photoca- } \\
\text { talysts/Reaction solu- } \\
\text { tion volume }\end{array}$ & Sacrificial agent & Light source & $\begin{array}{l}\text { Yields of product } \\
\quad\left(\mu \mathrm{mol} \mathrm{h} \mathrm{g}^{-1}\right)\end{array}$ & $\begin{array}{l}\text { Apparent quantum } \\
\text { yield (AQY) }\end{array}$ & References \\
\hline $\mathrm{MoS}_{2} / \mathrm{g}-\mathrm{C}_{3} \mathrm{~N}_{4}$ & $20 \mathrm{mg} / 100 \mathrm{~mL}$ & Lactic acid & $300 \mathrm{~W} \mathrm{Xe}(\lambda>420 \mathrm{~nm})$ & 1030 & $2.1 \%$ at $420 \mathrm{~nm}$ & {$[203]$} \\
\hline $\mathrm{TiO}_{2} / \mathrm{MoS}_{2}$ & $100 \mathrm{mg} / 100 \mathrm{~mL}$ & Methanol & $300 \mathrm{~W}$ Xe & 2145 & $6.4 \%$ at $360 \mathrm{~nm}$ & {$[40]$} \\
\hline CdS-NSs/rGO-WO $\mathrm{W}_{3}$ & $-/ 30 \mathrm{~mL}$ & $\mathrm{EtOH}$ & $300 \mathrm{~W} \mathrm{Xe}(\lambda>420 \mathrm{~nm})$ & 119.4 & $10.7 \%$ at $420 \mathrm{~nm}$ & {$[152]$} \\
\hline $\mathrm{g}-\mathrm{C}_{3} \mathrm{~N}_{4} / \mathrm{N}-\mathrm{La}_{2} \mathrm{Ti}_{2} \mathrm{O}_{7}$ & $5 \mathrm{mg} / 5 \mathrm{~mL}$ & Methanol & $500 \mathrm{~W}$ Xe & 430 & $2.1 \%$ at $420 \mathrm{~nm}$ & {$[105]$} \\
\hline $\mathrm{TiO}_{2} / \mathrm{g}-\mathrm{C}_{3} \mathrm{~N}_{4}$ & $10 \mathrm{mg} / 30 \mathrm{~mL}$ & TEOA & $300 \mathrm{~W} \mathrm{Xe}$ & 18,200 & $5.3 \%$ at $380 \mathrm{~nm}$ & {$[141]$} \\
\hline $\mathrm{ZnO}-\mathrm{MoS}_{2} / \mathrm{rGO}$ & $5 \mathrm{mg} /-$ & $\mathrm{Na}_{2} \mathrm{~S} / \mathrm{Na}_{2} \mathrm{SO}_{3}$ & $\begin{array}{c}\text { Natural } \\
\text { sunlight irradiation }\end{array}$ & 28,616 & - & [204] \\
\hline $\mathrm{CdS} / \mathrm{MoS}_{2}$ & $50 \mathrm{mg} / 80 \mathrm{~mL}$ & $\mathrm{Na}_{2} \mathrm{~S} / \mathrm{Na}_{2} \mathrm{SO}_{3}$ & $300 \mathrm{~W}$ Xe $(\lambda>400 \mathrm{~nm})$ & 8720 & - & {$[86]$} \\
\hline $\mathrm{Bi}_{4} \mathrm{Ti}_{3} \mathrm{O}_{12} / \mathrm{I}-\mathrm{BiOCl}$ & $50 \mathrm{mg} / 80 \mathrm{~mL}$ & Methanol & $350 \mathrm{~W}$ Xe $(\lambda>420 \mathrm{~nm})$ & 91.7 & - & {$[31]$} \\
\hline$a-\mathrm{Fe}_{2} \mathrm{O}_{3} / g-\mathrm{C}_{3} \mathrm{~N}_{4}$ & $10 \mathrm{mg} / 100 \mathrm{~mL}$ & TEOA & $300 \mathrm{~W}$ Xe $(\lambda>400 \mathrm{~nm})$ & $>30,000$ & $44.35 \%$ at $420 \mathrm{~nm}$ & {$[150]$} \\
\hline $\mathrm{CPFA} / \mathrm{g}-\mathrm{C}_{3} \mathrm{~N}_{4}$ & $100 \mathrm{mg} / 300 \mathrm{~mL}$ & Triethanol amine & $300 \mathrm{~W} \mathrm{Xe}(\lambda>400 \mathrm{~nm})$ & 584.7 & - & {$[205]$} \\
\hline $\mathrm{ZnIn}_{2} \mathrm{~S}_{4} / \mathrm{MoSe}_{2}$ & $5 \mathrm{mg} / 10 \mathrm{~mL}$ & Lactic acid & $300 \mathrm{~W}$ Xe $(\lambda>400 \mathrm{~nm})$ & 6454 & - & {$[206]$} \\
\hline Nickel boron oxide/Graphene & $40 \mathrm{mg} / 100 \mathrm{~mL}$ & TEOA & $300 \mathrm{~W}$ Xe $(\lambda>400 \mathrm{~nm})$ & $\sim 5000$ & - & {$[207]$} \\
\hline $\mathrm{MoS}_{2} / \mathrm{Cu}-\mathrm{ZnIn}_{2} \mathrm{~S}_{4}$ & $50 \mathrm{mg} / 250 \mathrm{~mL}$ & Ascorbic acid & $300 \mathrm{~W}$ Xe $(\lambda>420 \mathrm{~nm})$ & 5463 & $13.6 \%$ at $420 \mathrm{~nm}$ & {$[160]$} \\
\hline $\mathrm{MoS}_{2} / \mathrm{rGO}$ & $40 \mathrm{mg} / 250 \mathrm{~mL}$ & $\begin{array}{l}\text { TEOA and } \\
{[\mathrm{ZnTMPyP}]^{4+}}\end{array}$ & $300 \mathrm{~W}$ Xe $(\lambda>420 \mathrm{~nm})$ & 2560 & $15.2 \%$ at $420 \mathrm{~nm}$ & [208] \\
\hline $\mathrm{BP} / \mathrm{g}-\mathrm{C}_{3} \mathrm{~N}_{4}$ & $1.5 \mathrm{mg} / 40 \mathrm{~mL}$ & Methanol & $320 \mathrm{~W}$ Xe $(\lambda>420 \mathrm{~nm})$ & 427 & - & {$[108]$} \\
\hline $\mathrm{N}-\mathrm{ZnO} / \mathrm{g}-\mathrm{C}_{3} \mathrm{~N}_{4}$ & $5 \mathrm{mg} / 50 \mathrm{~mL}$ & $\mathrm{Na}_{2} \mathrm{~S} / \mathrm{Na}_{2} \mathrm{SO}_{3}$ & $320 \mathrm{~W}$ Xe & 18,836 & - & {$[52]$} \\
\hline $\mathrm{g}-\mathrm{C}_{3} \mathrm{~N}_{4} / \mathrm{MoS}_{2}$ & $50 \mathrm{mg} / 80 \mathrm{~mL}$ & Methanol & $350 \mathrm{~W}$ Xe $(\lambda>400 \mathrm{~nm})$ & 191.2 & - & [209] \\
\hline $\mathrm{Ni}_{2} \mathrm{P} / \mathrm{ZnIn}_{2} \mathrm{~S}_{4}$ & $50 \mathrm{mg} / 100 \mathrm{~mL}$ & Lactic acid & $300 \mathrm{~W} \mathrm{Xe}(\lambda>400 \mathrm{~nm})$ & 2066 & $7.7 \%$ at $420 \mathrm{~nm}$ & {$[112]$} \\
\hline $\mathrm{g}-\mathrm{C}_{3} \mathrm{~N}_{4} / \mathrm{ZnIn}_{2} \mathrm{~S}_{4}$ & $50 \mathrm{mg} / 60 \mathrm{~mL}$ & TEOA & $300 \mathrm{~W}$ Xe $(\lambda>420 \mathrm{~nm})$ & 2780 & $7.05 \%$ at $420 \mathrm{~nm}$ & {$[53]$} \\
\hline $\mathrm{Cu}_{y} / \mathrm{TiO}_{2} @ \mathrm{Ti}_{3} \mathrm{C}_{2} \mathrm{Tx}$ & $20 \mathrm{mg} / 150 \mathrm{~mL}$ & Methanol & $300 \mathrm{~W}$ Xe & 860 & - & {$[56]$} \\
\hline Phosphorene/g- $\mathrm{C}_{3} \mathrm{~N}_{4}$ & $20 \mathrm{mg} / 100 \mathrm{~mL}$ & Lactic acid & $300 \mathrm{~W}$ Xe $(\lambda>400 \mathrm{~nm})$ & 571 & $1.2 \%$ at $420 \mathrm{~nm}$ & {$[87]$} \\
\hline $\mathrm{g}-\mathrm{C}_{3} \mathrm{~N}_{4} / \mathrm{MgFe}$ & $30 \mathrm{mg} / 100 \mathrm{~mL}$ & Tricthanolamine & $300 \mathrm{~W} \mathrm{Xe}(\lambda>420 \mathrm{~nm})$ & 1260 & $6.9 \%$ at $420 \mathrm{~nm}$ & {$[210]$} \\
\hline $\mathrm{g}-\mathrm{C}_{3} \mathrm{~N}_{4} / \mathrm{MoS}_{2}$ & $3 \mathrm{mg} / 5 \mathrm{~mL}$ & Lactic acid & $350 \mathrm{~W}$ Xe $(\lambda>400 \mathrm{~nm})$ & 660 & $5.67 \%$ at $400 \mathrm{~nm}$ & {$[115]$} \\
\hline $\mathrm{g}-\mathrm{C}_{3} \mathrm{~N}_{4} / \mathrm{rGO}$ & $100 \mathrm{mg} / 100 \mathrm{~mL}$ & Tricthanolamine & $300 \mathrm{~W}$ Xe $(\lambda>420 \mathrm{~nm})$ & 715 & - & {$[211]$} \\
\hline $\mathrm{CoP} / \mathrm{g}-\mathrm{C}_{3} \mathrm{~N}_{4}$ & $50 \mathrm{mg} / 100 \mathrm{~mL}$ & Tricthanolamine & $300 \mathrm{~W}$ Xe $(\lambda>400 \mathrm{~nm})$ & $\sim 750$ & $4.3 \%$ at $420 \mathrm{~nm}$ & {$[212]$} \\
\hline $\mathrm{Fe}_{2} \mathrm{O}_{3} / g-\mathrm{C}_{3} \mathrm{~N}_{4}$ & $50 \mathrm{mg} / 80 \mathrm{~mL}$ & TEOA & $350 \mathrm{~W} \mathrm{Xe}(\lambda>420 \mathrm{~nm})$ & 398.0 & - & {$[92]$} \\
\hline $\mathrm{CoMoS}_{2} / \mathrm{rGO} / \mathrm{C}_{3} \mathrm{~N}_{4}$ & $100 \mathrm{mg} /-$ & TEOA & $300 \mathrm{~W}$ Xe $(\lambda>400 \mathrm{~nm})$ & 684 & - & {$[60]$} \\
\hline $\mathrm{CdS}-\mathrm{MoS}_{2} / \mathrm{rGO}-\mathrm{E}$ & $20 \mathrm{mg} / 80 \mathrm{~mL}$ & Lactic acid & $300 \mathrm{~W}$ Xe $(\lambda>420 \mathrm{~nm})$ & 36,700 & $30.5 \%$ at $420 \mathrm{~nm}$ & {$[213]$} \\
\hline $\mathrm{MoS}_{2} / \mathrm{CdS}$ & $50 \mathrm{mg} / 250 \mathrm{~mL}$ & $\mathrm{Na}_{2} \mathrm{~S} / \mathrm{Na}_{2} \mathrm{SO}_{3}$ & $300 \mathrm{~W}$ Xe $(\lambda>420 \mathrm{~nm})$ & 26,320 & $46.65 \%$ at $450 \mathrm{~nm}$ & {$[61]$} \\
\hline $\mathrm{g}-\mathrm{C}_{3} \mathrm{~N}_{4} /$ Graphene/ $\mathrm{MoS}_{2}$ & $50 \mathrm{mg} / 250 \mathrm{~mL}$ & TEOA & $300 \mathrm{~W} \mathrm{Xe}(\lambda>420 \mathrm{~nm})$ & 317 & $3.4 \%$ at $420 \mathrm{~nm}$ & {$[62]$} \\
\hline $\mathrm{CdS} / \mathrm{WS}_{2}$ & $3 \mathrm{mg} / 5 \mathrm{~mL}$ & Lactic acid & $350 \mathrm{~W}$ Xe & 14,100 & $70 \%$ at $460 \mathrm{~nm}$ & {$[113]$} \\
\hline $\mathrm{O}-\mathrm{g}-\mathrm{C}_{3} \mathrm{~N}_{4} / \mathrm{TiO}_{2}$ & $50 \mathrm{mg} / 50 \mathrm{~mL}$ & TEOA & $300 \mathrm{~W}$ Xe $(\lambda>400 \mathrm{~nm})$ & 587.1 & - & {$[142]$} \\
\hline $\begin{array}{c}\text { Phosphorus/Bismuth } \\
\text { vanadate }\end{array}$ & $5 \mathrm{mg} / 8 \mathrm{~mL}$ & - & $320 \mathrm{~W}$ Xe $(\lambda>420 \mathrm{~nm})$ & 160 & $0.89 \%$ at $420 \mathrm{~nm}$ & {$[214]$} \\
\hline $\mathrm{CdS} / \mathrm{WS}_{2} / \mathrm{g}-\mathrm{C}_{3} \mathrm{~N}_{4}$ & $10 \mathrm{mg} / 20 \mathrm{~mL}$ & TEOA & $300 \mathrm{~W} \mathrm{Xe}(\lambda>420 \mathrm{~nm})$ & 1174.5 & - & {$[215]$} \\
\hline $\mathrm{WO}_{3} / \mathrm{g}-\mathrm{C}_{3} \mathrm{~N}_{4}$ & $50 \mathrm{mg} / 80 \mathrm{~mL}$ & Lactic acid & $350 \mathrm{~W} \mathrm{Xe}$ & 982 & - & {$[116]$} \\
\hline $\mathrm{CuInS}_{2} / \mathrm{ZnIn}_{2} \mathrm{~S}_{4}$ & $50 \mathrm{mg} / 100 \mathrm{~mL}$ & $\mathrm{Na}_{2} \mathrm{~S} / \mathrm{Na}_{2} \mathrm{SO}_{3}$ & $300 \mathrm{~W}$ Xe $(\lambda>420 \mathrm{~nm})$ & 3430.2 & $12.4 \%$ at $420 \mathrm{~nm}$ & {$[67]$} \\
\hline $\begin{array}{l}\text { Phosphorus/Monolayer } \\
\qquad \mathrm{Bi}_{2} \mathrm{WO}_{6}\end{array}$ & $20 \mathrm{mg} / 100 \mathrm{~mL}$ & TEOA & $300 \mathrm{~W}$ Xe & 21042 & - & {$[153]$} \\
\hline $\mathrm{La}_{2} \mathrm{Ti}_{2} \mathrm{O}_{7} / \mathrm{In}_{2} \mathrm{~S}_{3}$ & $60 \mathrm{mg} / 100 \mathrm{~mL}$ & $\mathrm{Na}_{2} \mathrm{~S} / \mathrm{Na}_{2} \mathrm{SO}_{3}$ & $300 \mathrm{~W}$ Xe $(\lambda>400 \mathrm{~nm})$ & 158.89 & - & {$[144]$} \\
\hline $\mathrm{ZnIn}_{2} \mathrm{~S}_{4} / \mathrm{MoS}_{2}$ & $-/ 40 \mathrm{~mL}$ & Lactic acid & $300 \mathrm{~W}$ Xe $(\lambda>400 \mathrm{~nm})$ & 4974 & - & {$[117]$} \\
\hline $\mathrm{MoS}_{2} / \mathrm{SnNb}_{2} \mathrm{O}_{6}$ & $50 \mathrm{mg} / 50 \mathrm{~mL}$ & Methanol & $300 \mathrm{~W} \mathrm{Xe}(\lambda>420 \mathrm{~nm})$ & 258 & - & {$[70]$} \\
\hline $\mathrm{C}_{3} \mathrm{~N}_{4} / \mathrm{MoS}_{2}$ & $50 \mathrm{mg} / 100 \mathrm{~mL}$ & Methyl alcohol & $300 \mathrm{~W} \mathrm{Xe}$ & 385.04 & - & {$[216]$} \\
\hline $\mathrm{Ti}_{3} \mathrm{C}_{2}$ MXene/MoS 2 & $10 \mathrm{mg} /-$ & TEOA & $300 \mathrm{~W}$ Xe (AM 1.5) & 6425.297 & $4.61 \%$ at $420 \mathrm{~nm}$ & {$[75]$} \\
\hline g- $\mathrm{C}_{3} \mathrm{~N}_{4} / \mathrm{UMOFNs}$ & $15 \mathrm{mg} / 30 \mathrm{~mL}$ & Actic acid & $500 \mathrm{~W}$ Xe & 1909.02 & $2.34 \%$ at $405 \mathrm{~nm}$ & [217] \\
\hline
\end{tabular}


(Continued)

Photocatalytic hydrogen generation

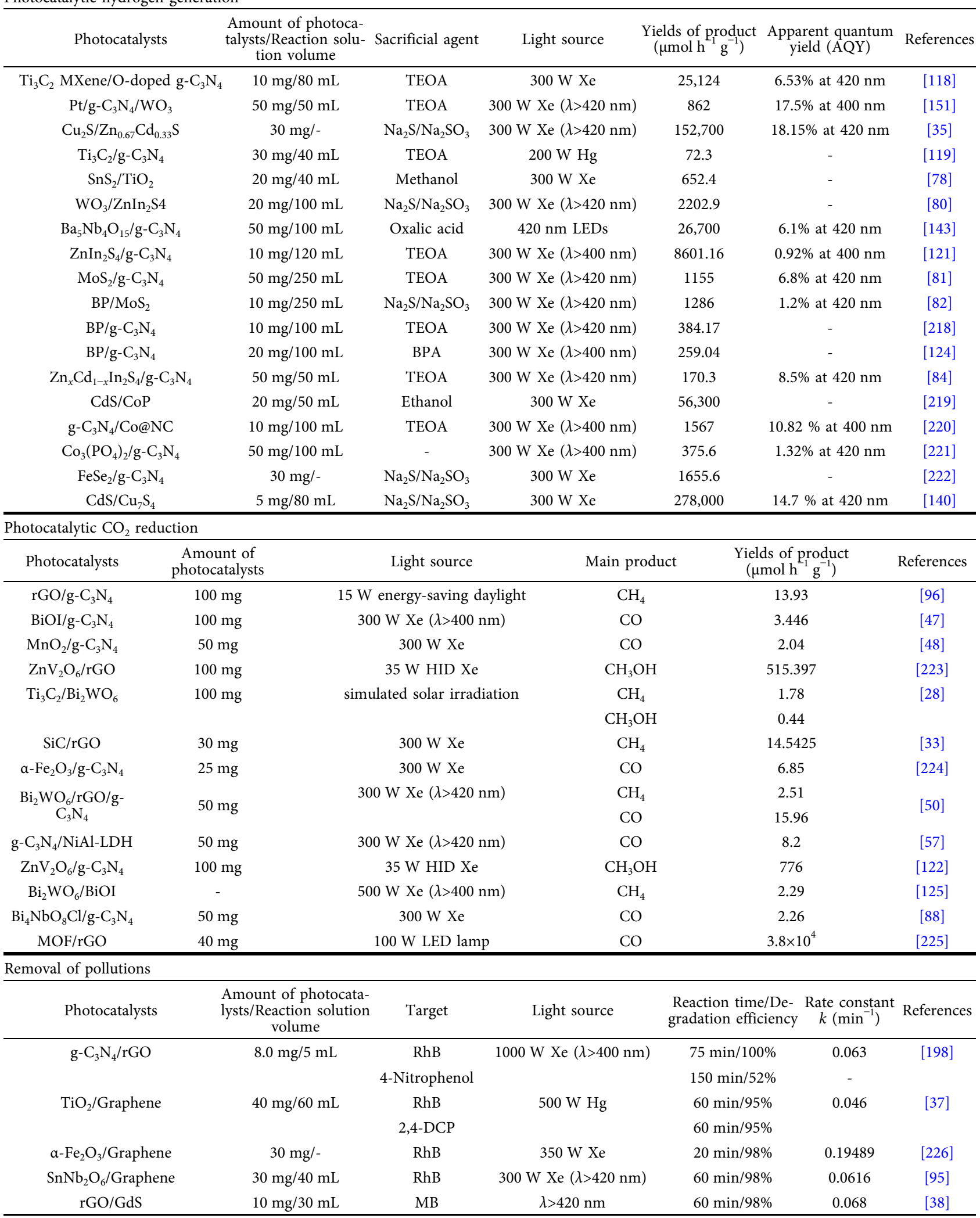




\begin{tabular}{|c|c|c|c|c|c|c|}
\hline \multicolumn{7}{|l|}{ Removal of pollutions } \\
\hline Photocatalysts & $\begin{array}{l}\text { Amount of photocata- } \\
\text { lysts/Reaction solution } \\
\text { volume }\end{array}$ & Target & Light source & $\begin{array}{l}\text { Reaction time/De- } \\
\text { gradation efficiency }\end{array}$ & $\begin{array}{l}\text { Rate constant } \\
k\left(\min ^{-1}\right)\end{array}$ & References \\
\hline $\mathrm{C}_{3} \mathrm{~N}_{4} / \mathrm{Bi}_{20} \mathrm{TiO}_{32}$ & $100 \mathrm{mg} / 100 \mathrm{~mL}$ & $\mathrm{RhB}$ & $300 \mathrm{~W}$ Xe $(\lambda>420 \mathrm{~nm})$ & $20 \mathrm{~min} / 98 \%$ & - & [29] \\
\hline Graphene $/ \mathrm{TiO}_{2}$ & $10 \mathrm{mg} / 30 \mathrm{~mL}$ & MB & $300 \mathrm{~W} \mathrm{Xe}(\lambda>420 \mathrm{~nm})$ & $3 \mathrm{~h} / \sim 80 \%$ & 0.00463 & [39] \\
\hline $\mathrm{BiOIO}_{3} / \mathrm{BiOI}$ & - & NO & $\lambda>420 \mathrm{~nm}$ & $30 \mathrm{~min} / 41.3 \%$ & - & [41] \\
\hline $\mathrm{SnS}_{2} / \mathrm{g}-\mathrm{C}_{3} \mathrm{~N}_{4}$ & $10 \mathrm{mg} / 100 \mathrm{~mL}$ & $\mathrm{RhB}$ & $300 \mathrm{~W}$ Xe $(\lambda>400 \mathrm{~nm})$ & $20 \mathrm{~min} / 99.8 \%$ & 0.2 & [97] \\
\hline $\mathrm{BiOBr} / \mathrm{La}_{2} \mathrm{Ti}_{2} \mathrm{O}_{7}$ & $40 \mathrm{mg} / 100 \mathrm{~mL}$ & $\mathrm{RhB}$ & $300 \mathrm{~W} \mathrm{Xe}$ & - & 0.092 & {$[45]$} \\
\hline $\mathrm{BiOCl} / \mathrm{La}_{2} \mathrm{Ti}_{2} \mathrm{O}_{7}$ & $40 \mathrm{mg} / 100 \mathrm{~mL}$ & $\mathrm{RhB}$ & $300 \mathrm{~W} \mathrm{Hg}$ & - & 0.19 & {$[46]$} \\
\hline CNX-NSs/rGO & $5 \mathrm{mg} / 10 \mathrm{~mL}$ & MB & $300 \mathrm{~W} \mathrm{Xe}$ & $40 \mathrm{~min} / 88 \%$ & - & [98] \\
\hline $\mathrm{P}-\mathrm{C}_{3} \mathrm{~N}_{4} / \mathrm{ZnIn}_{2} \mathrm{~S}_{4}$ & $30 \mathrm{mg} / 30 \mathrm{~mL}$ & 4-Nitroaniline & $300 \mathrm{~W} \mathrm{Xe}(\lambda>400 \mathrm{~nm})$ & $90 \mathrm{~min} / 99.4 \%$ & - & [42] \\
\hline $\mathrm{Bi}_{2} \mathrm{O}_{2} \mathrm{CO}_{3} / \mathrm{g}-\mathrm{C}_{3} \mathrm{~N}_{4}$ & $50 \mathrm{mg} / 50 \mathrm{~mL}$ & $\mathrm{RhB}$ & $500 \mathrm{~W} \mathrm{Xe}(\lambda>420 \mathrm{~nm})$ & $5 \mathrm{~h} / 74 \%$ & - & [99] \\
\hline $\mathrm{MoS}_{2} / \mathrm{g}-\mathrm{C}_{3} \mathrm{~N}_{4}$ & $40 \mathrm{mg} / 50 \mathrm{~mL}$ & $\mathrm{RhB}$ & $300 \mathrm{~W} \mathrm{Xe}(\lambda>420 \mathrm{~nm})$ & $20 \mathrm{~min} / 96 \%$ & 0.152 & {$[100]$} \\
\hline g- $\mathrm{C}_{3} \mathrm{~N}_{4} / \mathrm{Bi}_{4} \mathrm{O}_{5} \mathrm{I}_{2}$ & $500 \mathrm{mg} / 50 \mathrm{~mL}$ & $\mathrm{RhB}$ & $\lambda>420 \mathrm{~nm}$ & $40 \mathrm{~min} / 99 \%$ & 0.06 & [227] \\
\hline $\mathrm{C}_{3} \mathrm{~N}_{4} /$ Graphene & $20 \mathrm{mg} /-$ & $\mathrm{MO}$ & $300 \mathrm{~W} \mathrm{Xe}(\lambda>420 \mathrm{~nm})$ & $5 \mathrm{~h} / 73 \%$ & - & [103] \\
\hline $\mathrm{MoS}_{2} / \mathrm{g}-\mathrm{C}_{3} \mathrm{~N}_{4}$ & $25 \mathrm{mg} / 50 \mathrm{~mL}$ & MO & $300 \mathrm{~W} \mathrm{Xe}(\lambda>400 \mathrm{~nm})$ & $3 \mathrm{~h} / 74.4 \%$ & 0.461 & {$[101]$} \\
\hline $\mathrm{SnNb}_{2} \mathrm{O}_{6} / \mathrm{g}-\mathrm{C}_{3} \mathrm{~N}_{4}$ & $20 \mathrm{mg} / 80 \mathrm{~mL}$ & MB & $500 \mathrm{~W} W(\lambda>420 \mathrm{~nm})$ & $60 \mathrm{~min} / 55 \%$ & - & {$[102]$} \\
\hline $\mathrm{g}-\mathrm{C}_{3} \mathrm{~N}_{4} / \mathrm{N}-\mathrm{La}_{2} \mathrm{Ti}_{2} \mathrm{O}_{7}$ & $10 \mathrm{mg} / 10 \mathrm{~mL}$ & MO & $300 \mathrm{~W} \mathrm{Xe}(\lambda>400 \mathrm{~nm})$ & $3 \mathrm{~h} / 44 \%$ & - & {$[105]$} \\
\hline $\mathrm{g}-\mathrm{C}_{3} \mathrm{~N}_{4} / \mathrm{MgIn}_{2} \mathrm{~S}_{4}$ & $15 \mathrm{mg} / 30 \mathrm{~mL}$ & MO & $300 \mathrm{~W} \mathrm{Xe}(\lambda>400 \mathrm{~nm})$ & $70 \%$ & - & [228] \\
\hline $\mathrm{g}-\mathrm{C}_{3} \mathrm{~N}_{4} / \mathrm{TiO}_{2}$ & $10 \mathrm{mg} / 30 \mathrm{~mL}$ & MO & $300 \mathrm{~W}$ Xe & $15 \mathrm{~min} / 98 \%$ & 0.189 & [141] \\
\hline g- $\mathrm{C}_{3} \mathrm{~N}_{4} / \mathrm{Bi}_{4} \mathrm{O}_{5} \mathrm{Br}_{2}$ & $10 \mathrm{mg} / 100 \mathrm{~mL}$ & $\mathrm{RhB}$ & $300 \mathrm{~W} \mathrm{Xe}(\lambda>400 \mathrm{~nm})$ & $75 \mathrm{~min} / 91 \%$ & - & [229] \\
\hline $\mathrm{K}^{+} \mathrm{Ca}_{2} \mathrm{Nb}_{3} \mathrm{O}_{10}{ }^{-} / \mathrm{g}-\mathrm{C}_{3} \mathrm{~N}_{4}$ & $40 \mathrm{mg} / 40 \mathrm{~mL}$ & $\mathrm{TC}$ & $500 \mathrm{~W} W(\lambda>420 \mathrm{~nm})$ & $90 \mathrm{~min} / 80 \%$ & 0.0137 & {$[93]$} \\
\hline $\mathrm{WO}_{3} / \mathrm{K}^{+} \mathrm{Ca}_{2} \mathrm{Nb}_{3} \mathrm{O}_{10}^{-}$ & $40 \mathrm{mg} / 40 \mathrm{~mL}$ & $\mathrm{TC}$ & $250 \mathrm{~W} \mathrm{Xe}$ & $120 \mathrm{~min} / 85.8 \%$ & 0.0151 & [94] \\
\hline $\mathrm{WO}_{3} / \mathrm{SnNb}_{2} \mathrm{O}_{6}$ & $40 \mathrm{mg} / 40 \mathrm{~mL}$ & $\mathrm{RhB}$ & $500 \mathrm{~W}$ W & $180 \mathrm{~min} / 93.4 \%$ & 0.015 & {$[106]$} \\
\hline $\mathrm{Bi}_{4} \mathrm{Ti}_{3} \mathrm{O}_{12} / \mathrm{I}-\mathrm{BiOCl}$ & $50 \mathrm{mg} / 80 \mathrm{~mL}$ & MB & $350 \mathrm{~W} \mathrm{Xe}(\lambda>420 \mathrm{~nm})$ & $180 \mathrm{~min} / 90 \%$ & 0.013 & {$[31]$} \\
\hline $\mathrm{CeO}_{2} / \mathrm{BiOCl}$ & $20 \mathrm{mg} / 20 \mathrm{~mL}$ & $\mathrm{RhB}$ & direct sunlight & $60 \mathrm{~min} / 89 \%$ & - & [107] \\
\hline $\mathrm{g}-\mathrm{C}_{3} \mathrm{~N}_{4} / \mathrm{Bi}_{2} \mathrm{WO}_{6}$ & $10 \mathrm{mg} / 50 \mathrm{~mL}$ & Ibuprofen & $300 \mathrm{~W} \mathrm{Xe}(\lambda>420 \mathrm{~nm})$ & $60 \mathrm{~min} / 96.1 \%$ & 0.052 & [196] \\
\hline g- $\mathrm{C}_{3} \mathrm{~N}_{4} / \mathrm{MnO}_{2}$ & $50 \mathrm{mg} / 50 \mathrm{~mL}$ & Phenol & $300 \mathrm{~W}$ Xe & $180 \mathrm{~min} / 73.6 \%$ & 0.033 & [30] \\
\hline $\mathrm{Bi}_{2} \mathrm{~S}_{3}-\mathrm{BiOCl}$ & $10 \mathrm{mg} / 50 \mathrm{~mL}$ & $\mathrm{X}-3 \mathrm{~B}$ & $300 \mathrm{~W} \mathrm{Xe}(\lambda>400 \mathrm{~nm})$ & $30 \mathrm{~min} / 74.6 \%$ & 0.096 & [201] \\
\hline $\mathrm{Bi}_{2} \mathrm{WO}_{6} / \mathrm{TiO}_{2}$ & $10 \mathrm{mg} / 40 \mathrm{~mL}$ & 4-Nitroaniline & $300 \mathrm{~W}$ Xe & $16 \mathrm{~min} / 100 \%$ & - & [91] \\
\hline $\mathrm{TiO}_{2} / \mathrm{SnS}_{2}$ & $20 \mathrm{mg} / 100 \mathrm{~mL}$ & MB & $250 \mathrm{~W} \mathrm{Hg}$ & $60 \mathrm{~min} / 44 \%$ & - & {$[49]$} \\
\hline $\mathrm{ZnO} / \mathrm{V}_{2} \mathrm{O}_{5}$ & $10 \mathrm{mg} / 25 \mathrm{~mL}$ & MB & $300 \mathrm{~W} \mathrm{Xe}(\lambda>400 \mathrm{~nm})$ & $400 \mathrm{~min} / 90 \%$ & 0.0052 & {$[230]$} \\
\hline $\mathrm{C}_{3} \mathrm{~N}_{4}$-CdS & $500 \mathrm{mg} / 1000 \mathrm{~mL}$ & MO & $300 \mathrm{~W} \mathrm{Xe}$ & $210 \mathrm{~min} / 97 \%$ & - & [231] \\
\hline $\mathrm{Bi}_{3} \mathrm{O}_{4} \mathrm{Cl} / \mathrm{g}-\mathrm{C}_{3} \mathrm{~N}_{4}$ & $50 \mathrm{mg} / 100 \mathrm{~mL}$ & $\mathrm{TC}$ & $250 \mathrm{~W} \mathrm{Xe}(\lambda>420 \mathrm{~nm})$ & $60 \mathrm{~min} / 76 \%$ & 0.0205 & [197] \\
\hline $\mathrm{BiOIO}_{3} / \mathrm{g}-\mathrm{C}_{3} \mathrm{~N}_{4}$ & $-/ 50 \mathrm{~mL}$ & $\begin{array}{l}\text { 2,4,6-Trichloro- } \\
\text { phenol }\end{array}$ & $500 \mathrm{~W}$ Xe & $2.5 \mathrm{~h} / 92 \%$ & 0.016 & [109] \\
\hline $\mathrm{Bi}_{2} \mathrm{WO}_{6} / \mathrm{g}-\mathrm{C}_{3} \mathrm{~N}_{4}$ & $50 \mathrm{mg} / 100 \mathrm{~mL}$ & $\mathrm{RhB}$ & $500 \mathrm{~W} W(\lambda>420 \mathrm{~nm})$ & - & 0.043 & [111] \\
\hline $\mathrm{MoO}_{2} / \mathrm{g}-\mathrm{C}_{3} \mathrm{~N}_{4}$ & $50 \mathrm{mg} / 50 \mathrm{~mL}$ & $\mathrm{RhB}$ & $300 \mathrm{~W} \mathrm{Xe}(\lambda>420 \mathrm{~nm})$ & $120 \mathrm{~min} / 97.5 \%$ & - & [114] \\
\hline $\mathrm{N}-\mathrm{ZnO} / \mathrm{g}-\mathrm{C}_{3} \mathrm{~N}_{4}$ & $20 \mathrm{mg} / 50 \mathrm{~mL}$ & $\mathrm{RhB}$ & visible light irradiation & $180 \mathrm{~min} / 97 \%$ & 0.0089 & {$[51]$} \\
\hline $\mathrm{KTiNbO}_{5} / \mathrm{g}-\mathrm{C}_{3} \mathrm{~N}_{4}$ & $100 \mathrm{mg} /-$ & $\mathrm{RhB}$ & $300 \mathrm{~W} \mathrm{Xe}(\lambda>420 \mathrm{~nm})$ & $80 \mathrm{~min} / 89.9 \%$ & - & [232] \\
\hline $\mathrm{ZnO}-\mathrm{ZnCr}_{2} \mathrm{O}_{4} / \mathrm{g}-\mathrm{C}_{3} \mathrm{~N}_{4}-\mathrm{C}(\mathrm{N})$ & $50 \mathrm{mg} / 80 \mathrm{~mL}$ & Congo red & $500 \mathrm{~W} \mathrm{Xe}(\lambda>400 \mathrm{~nm})$ & $60 \mathrm{~min} / 70 \%$ & 0.0387 & [64] \\
\hline $\mathrm{SnNb}_{2} \mathrm{O}_{6} / \mathrm{CoFe}-\mathrm{LDH}$ & $50 \mathrm{mg} / 50 \mathrm{~mL}$ & $\mathrm{MO}$ & $500 \mathrm{~W} \mathrm{Xe}$ & $60 \mathrm{~min} / 83.3 \%$ & - & [54] \\
\hline $\mathrm{BiOI} / \mathrm{BiVO}_{4}$ & $30 \mathrm{mg} / 50 \mathrm{~mL}$ & $\mathrm{RhB}$ & Sunlamp $(\lambda>400 \mathrm{~nm})$ & $75 \mathrm{~min} / 97 \%$ & 0.0467 & [55] \\
\hline $\mathrm{g}-\mathrm{C}_{3} \mathrm{~N}_{4} / \mathrm{Bi}_{12} \mathrm{O}_{17} \mathrm{C}_{12}$ & $30 \mathrm{mg} / 50 \mathrm{~mL}$ & $\mathrm{RhB}$ & $300 \mathrm{~W} \mathrm{Xe}(\lambda>400 \mathrm{~nm})$ & $60 \mathrm{~min} / 90 \%$ & 0.353 & [65] \\
\hline $\mathrm{BiOCl} / \mathrm{g}-\mathrm{C}_{3} \mathrm{~N}_{4}$ & $50 \mathrm{mg} / 100 \mathrm{~mL}$ & 4-Chlorophenol & $300 \mathrm{~W} \mathrm{Xe}(\lambda>420 \mathrm{~nm})$ & $120 \mathrm{~min} / 95 \%$ & 0.025 & {$[58]$} \\
\hline $\mathrm{WC} / \mathrm{WO}_{3}$ & $20 \mathrm{mg} / 20 \mathrm{~mL}$ & $\mathrm{RhB}$ & $500 \mathrm{~W} \mathrm{Xe}(\lambda>400 \mathrm{~nm})$ & $-/ 89 \%$ & - & [202] \\
\hline $\mathrm{g}-\mathrm{C}_{3} \mathrm{~N}_{4} / \mathrm{rGO}$ & $-/ 20 \mathrm{~mL}$ & MO & $300 \mathrm{~W} \mathrm{Xe}(\lambda>400 \mathrm{~nm})$ & $180 \mathrm{~min} / 97 \%$ & - & [199] \\
\hline $\mathrm{Zn}_{3} \mathrm{In}_{2} \mathrm{~S}_{6} / \mathrm{F}-\mathrm{C}_{3} \mathrm{~N}_{4}$ & $40 \mathrm{mg} / 100 \mathrm{~mL}$ & $\mathrm{MO}$ & $300 \mathrm{~W} \mathrm{Xe}(\lambda>420 \mathrm{~nm})$ & $60 \mathrm{~min} / 99 \%$ & 0.07329 & {$[59]$} \\
\hline $\mathrm{GO} / \mathrm{g}-\mathrm{C}_{3} \mathrm{~N}_{4}$ & $20 \mathrm{mg} / 50 \mathrm{~mL}$ & $\mathrm{RhB}$ & $500 \mathrm{~W}$ Xe & - & 0.0514 & {$[110]$} \\
\hline
\end{tabular}




\begin{tabular}{|c|c|c|c|c|c|c|}
\hline \multicolumn{7}{|l|}{ Removal of pollutions } \\
\hline Photocatalysts & $\begin{array}{c}\text { Amount of photocata- } \\
\text { lysts/Reaction solution } \\
\text { volume }\end{array}$ & Target & Light source & $\begin{array}{l}\text { Reaction time/De- } \\
\text { gradation efficiency }\end{array}$ & $\begin{array}{c}\text { Rate constant } \\
k\left(\min ^{-1}\right)\end{array}$ & References \\
\hline $\mathrm{MoSe}_{2} / \mathrm{Bi}_{2} \mathrm{WO}_{6}$ & $100 \mathrm{mg} /-$ & Toluene & $300 \mathrm{~W} \mathrm{Xe}(\lambda>420 \mathrm{~nm})$ & $180 \mathrm{~min} / 80 \%$ & - & [233] \\
\hline $\mathrm{FeOCl} / \mathrm{GO}$ & $50 \mathrm{mg} / 100 \mathrm{~mL}$ & RhB & sunlight & $10 \mathrm{~min} / 100 \%$ & 0.32 & {$[234]$} \\
\hline $\mathrm{SnS}_{2} / \mathrm{CuInS}_{2}$ & $30 \mathrm{mg} / 100 \mathrm{~mL}$ & $\mathrm{MO}$ & $300 \mathrm{~W} \mathrm{Xe}(\lambda>400 \mathrm{~nm})$ & $60 \mathrm{~min} / 99 \%$ & - & {$[63]$} \\
\hline $\mathrm{BP} / \mathrm{g}-\mathrm{C}_{3} \mathrm{~N}_{4}$ & $20 \mathrm{mg} / 80 \mathrm{~mL}$ & $\mathrm{RhB}$ & $300 \mathrm{~W} \mathrm{Xe}(\lambda>420 \mathrm{~nm})$ & $30 \mathrm{~min} / 97 \%$ & 0.288 & [200] \\
\hline $\mathrm{Cu}_{2} \mathrm{WS}_{4} / \mathrm{g}-\mathrm{C}_{3} \mathrm{~N}_{4}$ & $50 \mathrm{mg} / 100 \mathrm{~mL}$ & $\begin{array}{c}\mathrm{Cr}(\mathrm{VI}) \\
\mathrm{TC}\end{array}$ & $300 \mathrm{~W} \mathrm{Xe}(\lambda>420 \mathrm{~nm})$ & $\begin{array}{l}100 \mathrm{~min} / 98.3 \% \\
120 \mathrm{~min} / 68.1 \%\end{array}$ & $\begin{array}{c}0.04076 \\
-\end{array}$ & [193] \\
\hline $\mathrm{Ag}-\mathrm{WO}_{3} / \mathrm{g}-\mathrm{C}_{3} \mathrm{~N}_{4}$ & $100 \mathrm{mg} / 300 \mathrm{~mL}$ & $\mathrm{RhB}$ & $500 \mathrm{~W} \mathrm{Xe}(\lambda>420 \mathrm{~nm})$ & $40 \mathrm{~min} / 96.2 \%$ & 0.053 & [235] \\
\hline $\mathrm{MnIn}_{2} \mathrm{~S}_{4} / \mathrm{g}-\mathrm{C}_{3} \mathrm{~N}_{4}$ & $30 \mathrm{mg} / 30 \mathrm{~mL}$ & $\mathrm{TC}$ & $300 \mathrm{~W} \mathrm{Xe}(\lambda>400 \mathrm{~nm})$ & $120 \mathrm{~min} / 100 \%$ & - & {$[66]$} \\
\hline $\mathrm{CuInS}_{2} / \mathrm{g}-\mathrm{C}_{3} \mathrm{~N}_{4}$ & $50 \mathrm{mg} / 100 \mathrm{~mL}$ & $\mathrm{TC}$ & $300 \mathrm{~W} \mathrm{Xe}(\lambda>420 \mathrm{~nm})$ & $60 \mathrm{~min} / 83.7 \%$ & 0.02583 & {$[68]$} \\
\hline $\mathrm{C}_{3} \mathrm{~N}_{4} / \mathrm{SnS}_{2}$ & $30 \mathrm{mg} / 30 \mathrm{~mL}$ & $\mathrm{MB}$ & LED light $(\lambda=410 \mathrm{~nm})$ & $30 \mathrm{~min} / 98.7 \%$ & 0.08258 & [69] \\
\hline $\mathrm{SnNb}_{2} \mathrm{O}_{6} / \mathrm{Bi}_{2} \mathrm{WO}_{6}$ & $50 \mathrm{mg} /-$ & $\begin{array}{c}\text { Quinolone anti- } \\
\text { biotic } \\
\text { Norfloxacin } \\
\text { (NOR) }\end{array}$ & $300 \mathrm{~W} \mathrm{Xe}(\lambda>420 \mathrm{~nm})$ & $60 \mathrm{~min} / \sim 90 \%$ & 0.0406 & {$[71]$} \\
\hline CQDs- $\mathrm{ZnIn}_{2} \mathrm{~S}_{4} / \mathrm{BiOCl}$ & $50 \mathrm{mg} / 100 \mathrm{~mL}$ & Antibiotics & $300 \mathrm{~W} \mathrm{Xe}(\lambda>420 \mathrm{~nm})$ & $120 \mathrm{~min} / 83.7 \%$ & 0.014 & {$[72]$} \\
\hline $\mathrm{CoAl}-\mathrm{LDH} / \mathrm{g}-\mathrm{C}_{3} \mathrm{~N}_{4} / \mathrm{rGO}$ & $50 \mathrm{mg} / 200 \mathrm{~mL}$ & $\begin{array}{c}\text { Congo red } \\
\text { TC }\end{array}$ & $300 \mathrm{~W}$ halogen & $\begin{array}{l}30 \mathrm{~min} / 99 \% \\
60 \mathrm{~min} / 99 \%\end{array}$ & $\begin{array}{l}- \\
-\end{array}$ & {$[236]$} \\
\hline Carbon dots- $\mathrm{BiVO}_{4} / \mathrm{Bi}_{3} \mathrm{TaO}_{7}$ & $10 \mathrm{mg} / 30 \mathrm{~mL}$ & $\mathrm{TC}$ & $500 \mathrm{~W} \mathrm{Xe}(\lambda>420 \mathrm{~nm})$ & $120 \mathrm{~min} / 85.3 \%$ & - & [237] \\
\hline $\mathrm{MoS}_{2} / \mathrm{PbS}$ & $25 \mathrm{mg} / 250 \mathrm{~mL}$ & MB & $300 \mathrm{~W} \mathrm{Xe}(\lambda>420 \mathrm{~nm})$ & $48 \mathrm{~min} / 90 \%$ & 0.0431 & {$[74]$} \\
\hline $\mathrm{Bi} @ \mathrm{Bi}_{5} \mathrm{O}_{7} \mathrm{I} / \mathrm{rGO}$ & $30 \mathrm{mg} /-$ & Levofloxacin & $300 \mathrm{~W} \mathrm{Xe}(\lambda>420 \mathrm{~nm})$ & $60 \mathrm{~min} / 87.7 \%$ & 0.0322 & [238] \\
\hline $\mathrm{BiOCl} / \mathrm{K}^{+} \mathrm{Ca}_{2} \mathrm{Nb}_{3} \mathrm{O}_{10}{ }^{-}$ & $35 \mathrm{mg} / 35 \mathrm{~mL}$ & $\mathrm{TC}$ & $250 \mathrm{~W}$ Xe & $150 \mathrm{~min} / 94.5 \%$ & 0.01568 & {$[76]$} \\
\hline $\mathrm{CdIn}_{2} \mathrm{~S}_{4} / \mathrm{N}-\mathrm{rGO}$ & $50 \mathrm{mg} / 100 \mathrm{~mL}$ & 2,4-DCP & $\lambda>420 \mathrm{~nm}$ & $6 \mathrm{~h} / 70 \%$ & 0.0044 & {$[77]$} \\
\hline $\mathrm{SnS}_{2} / \mathrm{g}-\mathrm{C}_{3} \mathrm{~N}_{4}$ & $10 \mathrm{mg} / 50 \mathrm{~mL}$ & $\mathrm{RhB}$ & $300 \mathrm{~W} \mathrm{Xe}(\lambda>400 \mathrm{~nm})$ & $60 \mathrm{~min} / 94.8 \%$ & 0.0302 & {$[123]$} \\
\hline $\mathrm{BiVO}_{4} / \mathrm{g}-\mathrm{C}_{3} \mathrm{~N}_{4}$ & $10 \mathrm{mg} / 20 \mathrm{~mL}$ & $\mathrm{RhB}$ & $300 \mathrm{~W} \mathrm{Xe}(\lambda>420 \mathrm{~nm})$ & $60 \mathrm{~min} / 100 \%$ & 0.0410 & {$[120]$} \\
\hline $\mathrm{Wg}-\mathrm{C}_{3} \mathrm{~N}_{4} / \mathrm{g}-\mathrm{C}_{3} \mathrm{~N}_{4}$ & $350 \mathrm{mg} / 125 \mathrm{~mL}$ & $\mathrm{AV}-7$ & fluorescent & $30 \mathrm{~min} / 96 \%$ & 0.0809 & [239] \\
\hline $\mathrm{TiO}_{2} / \mathrm{WS}_{2}$ & $20 \mathrm{mg} / 100 \mathrm{~mL}$ & $\mathrm{RhB}$ & $300 \mathrm{~W} \mathrm{Xe}(\lambda>420 \mathrm{~nm})$ & $90 \mathrm{~min} / 100 \%$ & - & [79] \\
\hline rGO-BWO & $20 \mathrm{mg} / 100 \mathrm{~mL}$ & $\mathrm{TC}$ & - & $60 \mathrm{~min} / 85.0 \%$ & 0.030 & {$[80]$} \\
\hline $\mathrm{SnS}_{2} / \mathrm{MoS}_{2}$ & $25 \mathrm{mg} / 100 \mathrm{~mL}$ & $\mathrm{MB}$ & $250 \mathrm{~W} \mathrm{Hg}$ & $60 \mathrm{~min} / 100 \%$ & 0.00937 & {$[83]$} \\
\hline $\mathrm{Ni}(\mathrm{OH})_{2} / \mathrm{Bi}_{4} \mathrm{Ti}_{3} \mathrm{O}_{12}$ & $20 \mathrm{mg} / 100 \mathrm{~mL}$ & Levofloxacin & $300 \mathrm{~W}$ Xe & $80 \mathrm{~min} / 62 \%$ & 0.01152 & [89] \\
\hline $\mathrm{CdS} / \mathrm{g}-\mathrm{C}_{3} \mathrm{~N}_{4}$ & $50 \mathrm{mg} / 50 \mathrm{~mL}$ & $\mathrm{RhB}$ & $500 \mathrm{~W} \mathrm{Xe}(\lambda>420 \mathrm{~nm})$ & $120 \mathrm{~min} / 96.5 \%$ & 0.02414 & {$[85]$} \\
\hline $\mathrm{In}_{2} \mathrm{~S}_{3} / \mathrm{Bi}_{2} \mathrm{O}_{2} \mathrm{CO}_{3}$ & $30 \mathrm{mg} / 30 \mathrm{~mL}$ & $\mathrm{RhB}$ & $400 \mathrm{~W} \mathrm{Xe}$ & $60 \mathrm{~min} / 91 \%$ & 0.035 & {$[156]$} \\
\hline
\end{tabular}

Abbreviations: AV-7: acid violet-7; 2,4-DCP: 2,4-dichlorophenol; LED: light-emitted diode; MB: methylene blue; MO: methyl orange; TEOA: triethanolamine; UMOFNs: ultrathinning metal-organic frameworks

subjected to low photocatalytic efficiency, which is far from enough to meet the requirements of practical applications. Generally, there are several shortcomings responsible for the low photocatalytic efficiency of the current photocatalysts, such as rapid recombination of photogenerated charge carriers, poor light harvesting and inactive charge transport kinetics [140].

Recent studies regarding 2D/2D heterojunction materials found that these composite photocatalysts with properly engineered energy band matching and preferable dimensionality design are quite promising to overcome the above-mentioned shortcomings in conventional photocatalysts, thus reaching the excellent hydrogen evolution efficiency [140]. Many advanced 2D/2D heterojunction photocatalysts have been explored to split water for hydrogen generation as summarized in Table 3. Typically, the advanced 2D/2D composite semiconductors can be classified into five types, including Type-I, Type-II, Z-scheme, S-scheme and cocatalyst/ photocatalyst (Fig. 9).

In a typical $2 \mathrm{D} / 2 \mathrm{D}$ Type-I configuration (Fig. 9a), the valence band $(\mathrm{VB})$ and conduction band $(\mathrm{CB})$ of one $2 \mathrm{D}$ semiconductor (marked as B) are located within the band gap of another 2D semiconductor (marked as A). Thus, semiconductor A is often mainly responsible for harvesting light to produce electrons and holes, while semi- 
a

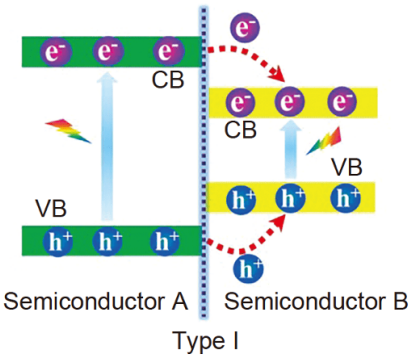

b

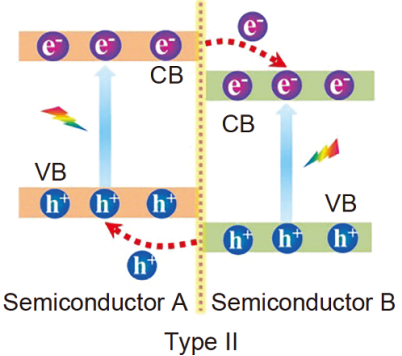

e

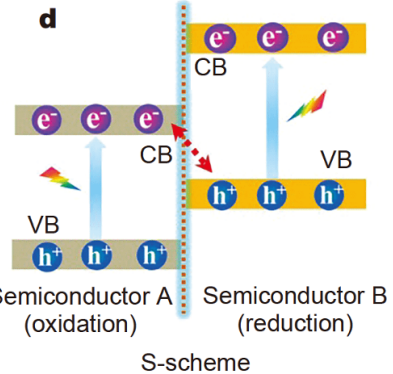

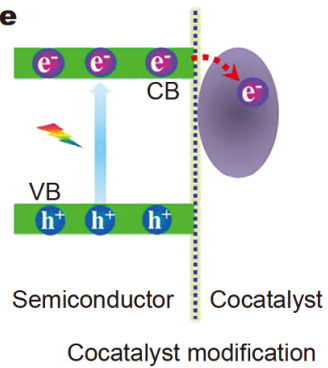

Figure 9 Schematic diagrams of the five types of heterojunctions.

conductor B acts as electron as well as hole acceptors to promote the charge transfer. In 2017, Zhu et al. [108] developed an efficient metal-free 2D/2D heterojunction photocatalyst with Type-I configuration for photocatalytic $\mathrm{H}_{2}$ evolution. The $2 \mathrm{D} / 2 \mathrm{D}$ nanohybrid was composed of $\mathrm{BP}$ nanoflakes and $\mathrm{g}-\mathrm{C}_{3} \mathrm{~N}_{4}(\mathrm{CN})$ nanosheets. In this photocatalyst system, the $\mathrm{CN}$ was mainly excited to generate electrons and holes under visible light irradiation, while BP acted as an electron and hole acceptor from adjacent $\mathrm{CN}$ to inhibit the recombination of the photogenerated charges in $\mathrm{CN}$ (Fig. 10a). As a result, the 2D/2D CN/BP photocatalytic system with an optimal ratio of $\mathrm{BP}: \mathrm{CN}$ at 1:4 presented a higher $\mathrm{H}_{2}$ evolution rate of $427 \mu \mathrm{mol} \mathrm{g}^{-1} \mathrm{~h}^{-1}$. Another interesting 2D/2D metalfree photocatalyst with Type-I configuration was reported by Qiao's group [87]. They prepared a novel 2D/2D phosphorene/g- $\mathrm{C}_{3} \mathrm{~N}_{4}$ vdW heterojunction, which exhibited an enhanced visible-light photocatalytic $\mathrm{H}_{2}$ production activity of $571 \mu \mathrm{mol} \mathrm{g}^{-1} \mathrm{~h}^{-1}$. 2D chalcogenide material can also be combined with $\mathrm{g}-\mathrm{C}_{3} \mathrm{~N}_{4}$ to construct 2D/2D Type-I heterojunction for excellent photocatalytic $\mathrm{H}_{2}$ evolution. Lin et al. [53] reported $2 \mathrm{D} / 2 \mathrm{D} \mathrm{g}-\mathrm{C}_{3} \mathrm{~N}_{4}$ nanosheet@ZnIn $\mathrm{S}_{4}$ nanoleaf for photocatalytic $\mathrm{H}_{2}$ generation. According to the Type-I charge transfer mechanism (Fig. 10b), the photoinduced CB electrons and VB holes on the g- $\mathrm{C}_{3} \mathrm{~N}_{4}$ nanosheets could readily transfer to the $\mathrm{CB}$ and VB of $\mathrm{ZnIn}_{2} \mathrm{~S}_{4}$, respectively, contributing to fairly high photoinduced charge separation and migration efficiency. Furthermore, the unusual 2D/2D heterojunction geometry endowed the heterojunction system with highspeed charge transfer nanochannels, ultimately resulting in a remarkable visible-light-driven $\mathrm{H}_{2}$ evolution rate of $2780 \mu \mathrm{mol} \mathrm{g}^{-1} \mathrm{~h}^{-1}$. More recently, Yang et al. [121] made further progress by using $2 \mathrm{D} / 2 \mathrm{D}$ g- $\mathrm{C}_{3} \mathrm{~N}_{4} / \mathrm{ZnIn}_{2} \mathrm{~S}_{4}$ Type-I heterojunction for photocatalytic $\mathrm{H}_{2}$ evolution. In this study, they fabricated $2 \mathrm{D} / 2 \mathrm{D}$ ultra-thin $\mathrm{ZnIn}_{2} \mathrm{~S}_{4}$ /protonated $\mathrm{g}_{-} \mathrm{C}_{3} \mathrm{~N}_{4}$ nanocomposites, which showed excellent photocatalytic $\mathrm{H}_{2}$ production rate of $8601.16 \mu \mathrm{mol} \mathrm{g}^{-1} \mathrm{~h}^{-1}$ under visible light irradiation.

Type-II composite structures consist of two semiconductors with a staggered energy band alignment (Fig. 9b). During the photocatalytic process, the photoinduced electrons in the $\mathrm{CB}$ of semiconductor $\mathrm{A}$ transfer to the $\mathrm{CB}$ of semiconductor $\mathrm{B}$, while the holes in the $\mathrm{VB}$ of $\mathrm{A}$ are transferred to the $\mathrm{VB}$ of $\mathrm{B}$, which is derived by the potential between them. Compared with conventional Type-II heterojunctions, 2D Type-II heterojunctions own unique features in photocatalytic applications. The faceto-face manner not only offers the most charge transportation pathways but also ensures a very short migration distance for photogenerated charges, leading to a boosted photocatalytic performance. Gu et al. [141] reported a face-to-face interfacial assembly of $\mathrm{TiO}_{2} / \mathrm{g}-\mathrm{C}_{3} \mathrm{~N}_{4}$ Type-II hybrid for photocatalytic hydrogen evolution. Under UV-Vis light irradiation, both $\mathrm{g}_{-} \mathrm{C}_{3} \mathrm{~N}_{4}$ and $\mathrm{TiO}_{2}$ can be excited by photon energy higher than their bandgaps. Then, the photoinduced electrons of the $\mathrm{CB}$ in g$\mathrm{C}_{3} \mathrm{~N}_{4}$ would drill into the $\mathrm{CB}$ of $\mathrm{TiO}_{2}$, while the photo- 

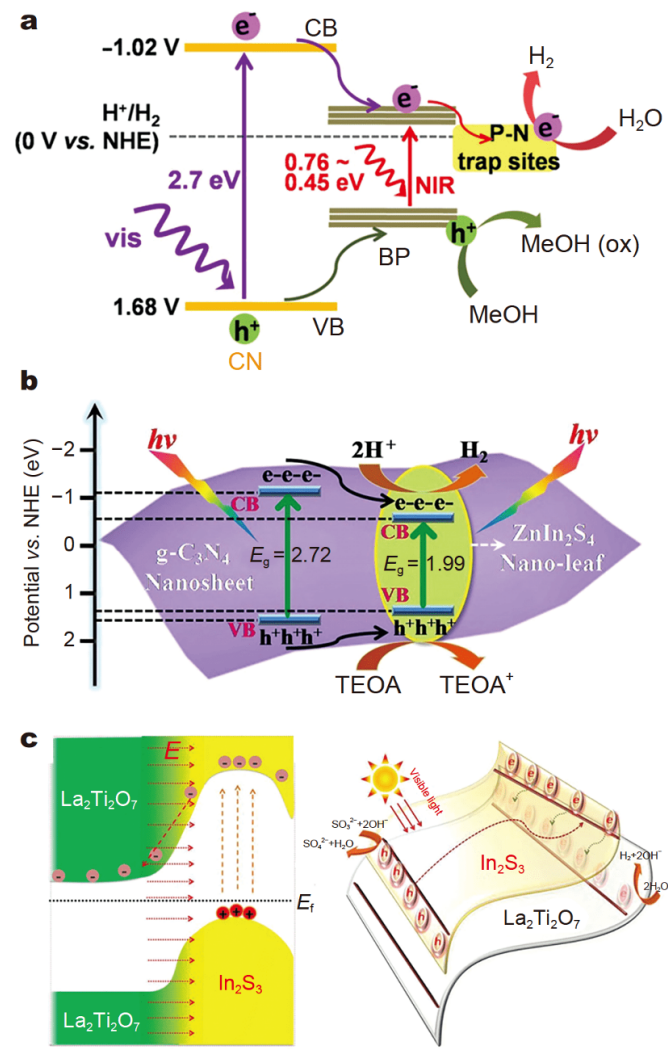

Figure 10 (a) Schematic diagram for the photocatalytic $\mathrm{H}_{2}$ evolution using BP/CN photocatalyst. Reprinted with permission from Ref. [108]. Copyright 2017, American Chemical Society. (b) Schematic diagram for the photocatalytic $\mathrm{H}_{2}$ evolution using $2 \mathrm{D} / 2 \mathrm{D}$ g- $\mathrm{C}_{3} \mathrm{~N}_{4} / \mathrm{ZnIn}_{2} \mathrm{~S}_{4}$ photocatalyst. NHE: normal hydrogen electrode. Reprinted with permission from Ref. [53]. Copyright 2018, Elsevier. (c) Schematic diagram for the photocatalytic $\mathrm{H}_{2}$ evolution using $\mathrm{La}_{2} \mathrm{Ti}_{2} \mathrm{O}_{7} / \mathrm{In}_{2} \mathrm{~S}_{3}$ photocatalyst. Reprinted with permission from Ref. [144]. Copyright 2019, Elsevier.

induced holes of the $\mathrm{VB}$ in $\mathrm{TiO}_{2}$ will transfer to the $\mathrm{VB}$ of $\mathrm{g}_{-} \mathrm{C}_{3} \mathrm{~N}_{4}$. Due to the unique, ultrathin $2 \mathrm{D}$ face-to-face contact features, the photoinduced charge carriers would travel a very short distance between $\mathrm{g}-\mathrm{C}_{3} \mathrm{~N}_{4}$ and $\mathrm{TiO}_{2}$, resulting in a high photocatalytic hydrogen evolution rate of $18,200 \mu \mathrm{mol} \mathrm{g}^{-1} \mathrm{~h}^{-1}$. Later, Zhong and coworkers [142] prepared a $2 \mathrm{D} / 2 \mathrm{D} \mathrm{O}-\mathrm{g}-\mathrm{C}_{3} \mathrm{~N}_{4} / \mathrm{TiO}_{2}$ composite with TypeII configuration for visible-light-driven photocatalytic hydrogen evolution. Some perovskite-type 2D semiconductors with a hexagonal crystal structure can also be coupled with $2 \mathrm{D} \mathrm{g}-\mathrm{C}_{3} \mathrm{~N}_{4}$ to form a $2 \mathrm{D} / 2 \mathrm{D}$ Type-II photocatalyst for photocatalytic hydrogen evolution. Cai et al. [105] reported the hybridization of $7 \mathrm{~nm}$ thick $\mathrm{N}$-doped $\mathrm{La}_{2} \mathrm{Ti}_{2} \mathrm{O}_{7}$ (NLTO) nanosheets with $2 \mathrm{~nm}$ thick g- $\mathrm{C}_{3} \mathrm{~N}_{4}$ nanosheets to construct a g- $\mathrm{C}_{3} \mathrm{~N}_{4} / \mathrm{NLTO}$ composite with a Type-II band alignment, in which the $\mathrm{g}^{-} \mathrm{C}_{3} \mathrm{~N}_{4}$ and NLTO respectively act as hole receptor and electron conductor. As a result, the optimal g- $\mathrm{C}_{3} \mathrm{~N}_{4} / \mathrm{NLTO}$ photocatalyst exhibited a $\mathrm{H}_{2}$ evolution rate of $430 \mu \mathrm{mol} \mathrm{g}^{-1} \mathrm{~h}^{-1}$, which was 10 and 2 times higher than those of LTO and NLTO, respectively. More recently, Wang et al. [143] employed another layered perovskite semiconductor $\left(\mathrm{Ba}_{5} \mathrm{Nb}_{4} \mathrm{O}_{15}\right)$ to combine with $\mathrm{g}-\mathrm{C}_{3} \mathrm{~N}_{4}$ to form a 2D/2D Type-II heterojunction towards enhanced photocatalytic activity. The $\mathrm{Ba}_{5} \mathrm{Nb}_{4} \mathrm{O}_{15} / \mathrm{g}-\mathrm{C}_{3} \mathrm{~N}_{4}$ photocatalyst exhibited a higher hydrogen evolution rate of $2,670 \mu \mathrm{mol} \mathrm{g}{ }^{-1} \mathrm{~h}^{-1}$, which was 2.35 times that of $\mathrm{g}-\mathrm{C}_{3} \mathrm{~N}_{4}$. Layered perovskite semiconductors have also been reported to couple with other $2 \mathrm{D}$ materials to form $2 \mathrm{D} / 2 \mathrm{D}$ heterojunctions with Type-II configuration. For instance, Qian et al. [31] designed $\mathrm{Bi}_{4} \mathrm{Ti}_{3} \mathrm{O}_{12} / \mathrm{I}-\mathrm{BiOCl} 2 \mathrm{D} / 2 \mathrm{D}$ heterojunction systems for photocatalytic hydrogen production. In another study, Hua and coworkers [144] fabricated a Type-II $\mathrm{La}_{2} \mathrm{Ti}_{2} \mathrm{O}_{7} / \mathrm{In}_{2} \mathrm{~S}_{3}$ heterojunction. As shown in Fig. $10 \mathrm{c}$, the $\mathrm{La}_{2} \mathrm{Ti}_{2} \mathrm{O}_{7} / \mathrm{In}_{2} \mathrm{~S}_{3}$ nanosheet heterojunction possessed intimate face-to-face contact feature between them, which guarantees facile electron migrations from $\mathrm{In}_{2} \mathrm{~S}_{3}$ to $\mathrm{La}_{2} \mathrm{Ti}_{2} \mathrm{O}_{7}$. Consequencely, the $\mathrm{La}_{2} \mathrm{Ti}_{2} \mathrm{O}_{7} / \mathrm{In}_{2} \mathrm{~S}_{3}$ nanosheet heterojunctions demonstrated a much improved photocatalytic hydrogen production compared with pristine $\mathrm{In}_{2} \mathrm{~S}_{3}$ as well as $\mathrm{La}_{2} \mathrm{Ti}_{2} \mathrm{O}_{7}$.

Z-scheme $2 \mathrm{D} / 2 \mathrm{D}$ photocatalysts (Fig. 9c) can not only hold the attractive $2 \mathrm{D} / 2 \mathrm{D}$ contact mode but also benefit from the Z-scheme photocatalytic system which can attain higher redox capacities than the traditional heterojunction [145-149]. She et al. [150] synthesized an $\alpha-$ $\mathrm{Fe}_{2} \mathrm{O}_{3} / g-\mathrm{C}_{3} \mathrm{~N}_{4} 2 \mathrm{D} / 2 \mathrm{D} \mathrm{Z}$-scheme hybrid photocatalyst, in which the $\mathrm{CB}$ electrons of $\alpha-\mathrm{Fe}_{2} \mathrm{O}_{3}$ could easily jump to the $\mathrm{VB}$ of $\mathrm{g}-\mathrm{C}_{3} \mathrm{~N}_{4}$ and recombine with the photoinduced holes in the VB of $\mathrm{g}_{-} \mathrm{C}_{3} \mathrm{~N}_{4}$ (Fig. 11a). As a result, the electron-hole recombination in both $\alpha-\mathrm{Fe}_{2} \mathrm{O}_{3}$ and g- $\mathrm{C}_{3} \mathrm{~N}_{4}$ were dramatically suppressed and the $2 \mathrm{D} / 2 \mathrm{D}$ tight interface could pave the efficient transfer of the photoexcited electron to the reactant. Under visible light irradiation, the $\alpha-\mathrm{Fe}_{2} \mathrm{O}_{3} / g-\mathrm{C}_{3} \mathrm{~N}_{4}$ heterojunction displayed a very higher hydrogen production rate of $31,400 \mu \mathrm{mol} \mathrm{g}^{-1} \mathrm{~h}^{-1}$ (Fig. 11b). Soon afterwards, $\mathrm{Xu}$ and coworkers [92] also prepared an $\alpha-\mathrm{Fe}_{2} \mathrm{O}_{3} / g-\mathrm{C}_{3} \mathrm{~N}_{4} 2 \mathrm{D} / 2 \mathrm{D}$ heterojunction photocatalyst, again confirming the $\mathrm{Z}$ scheme charge transfer route and $2 \mathrm{D} / 2 \mathrm{D}$ structure advantages for effcient photocatalytic $\mathrm{H}_{2}$ evolution. Another g- $\mathrm{C}_{3} \mathrm{~N}_{4}$-based $2 \mathrm{D} / 2 \mathrm{D} \mathrm{Z}$-scheme photocatalyst was fabricated by incorporating hydrogen-treated $\mathrm{WO}_{3}$ nanosheets (HWO) to Pt-loaded g- $\mathrm{C}_{3} \mathrm{~N}_{4}$ nanosheets (Pt-CN) [151], and used for efficiently catalyzing the $\mathrm{H}_{2}$ generation reaction. Similarly, $2 \mathrm{D} \mathrm{WO}_{3}$ nanosheets were also employed to couple with some $2 \mathrm{D}$ chalcogenides to form $2 \mathrm{D} / 2 \mathrm{D} \mathrm{Z}$ - 

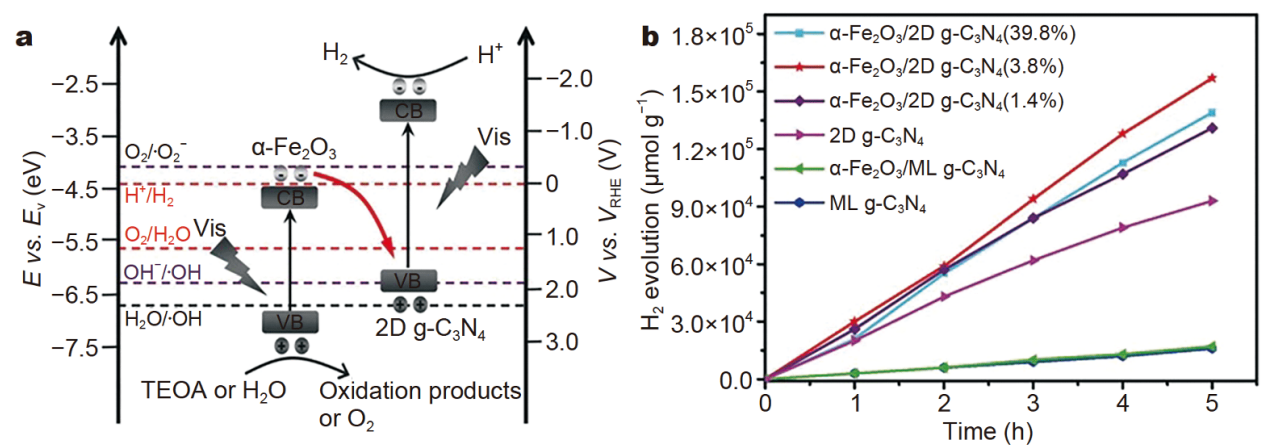

c

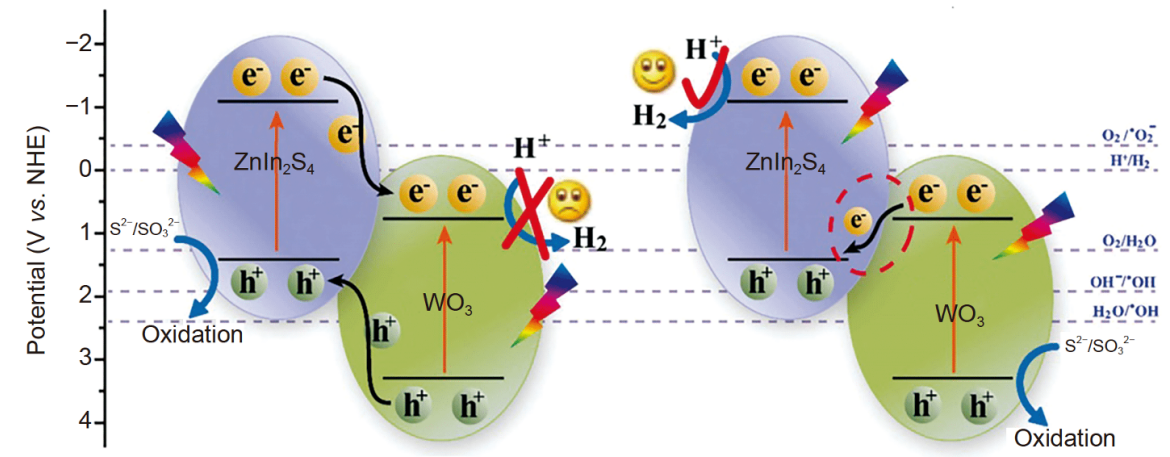

Double-transfer mechanism

Z-scheme mechanism
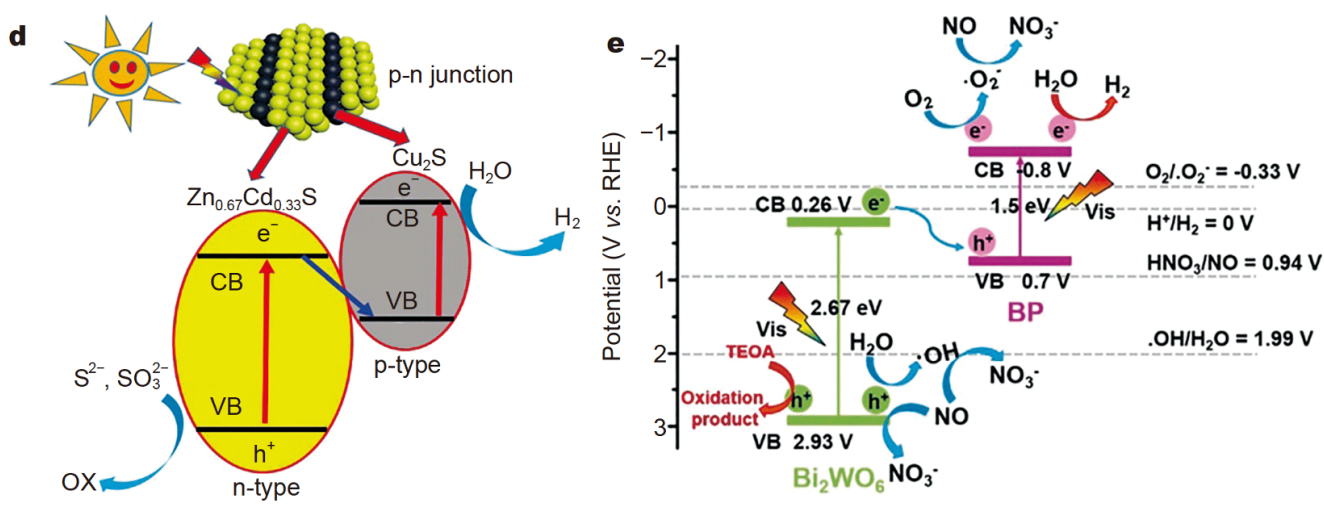

Figure 11 (a) Z-scheme mechanism in $\alpha-\mathrm{Fe}_{2} \mathrm{O}_{3} / g-\mathrm{C}_{3} \mathrm{~N}_{4}$ hybrids. (b) Photocatalytic $\mathrm{H}_{2}$ evolution over $\alpha$ - $\mathrm{Fe}_{2} \mathrm{O}_{3} / g$ - $\mathrm{C}_{3} \mathrm{~N}_{4}$ hybrids. Reprinted with permission from Ref. [150]. Copyright 2017, Wiley-VCH. (c) Schematic diagrams of mechanisms for photocatalytic $\mathrm{H}_{2}$ evolution over $\mathrm{WO}_{3} / \mathrm{ZnIn}_{2} \mathrm{~S}_{4}$ samples. Reprinted with permission from Ref. [90]. Copyright 2019, Royal Society of Chemistry. (d) Schematic of the photocatalytic water splitting over the $2 \mathrm{D} / 2 \mathrm{D} \mathrm{Cu} \mathrm{S}_{2} / \mathrm{Zn}_{0.67} \mathrm{Cd}_{0.33} \mathrm{~S}$. Reprinted with permission from Ref. [35]. Copyright 2019, Royal Society of Chemistry. (e) Z-scheme mechanism in $\mathrm{BP} / \mathrm{Bi}_{2} \mathrm{WO}_{6}$ hybrids. Reprinted with permission from Ref. [153]. Copyright 2019, Wiley-VCH.

scheme photocatalysts. Huang et al. [152] reported the combination of CdS nanosheets or CdS/rGO nanosheets with ultrathin $\mathrm{WO}_{3}$ nanosheets to fabricate $2 \mathrm{D} / 2 \mathrm{D} \mathrm{Z}$ scheme photocatalytic systems. In water-ethanol mixtures, the as-synthesized $\mathrm{CdS} / \mathrm{WO}_{3}$ and $\mathrm{CdS} / \mathrm{rGO}-\mathrm{WO}_{3}$ composites demonstrated higher photocatalytic hydrogen evolution activities than pure ultrathin 2D CdS nanosheets. In another study, Tan and coworkers [90] used $2 \mathrm{D} \mathrm{WO}_{3}$ nanosheets to couple with a ternary $2 \mathrm{D}$ chal- cogenide to prepare a Z-scheme $\mathrm{WO}_{3} / \mathrm{ZnIn}_{2} \mathrm{~S}_{4} 2 \mathrm{D} / 2 \mathrm{D}$ composite (Fig. 11c), which exhibited a high $\mathrm{H}_{2}$ production rate of $2,202.9 \mu \mathrm{mol} \mathrm{g} \mathrm{h}^{-1}$. Combining different $2 \mathrm{D}$ chalcogenides can also be used to form the $2 \mathrm{D} / 2 \mathrm{D} \mathrm{Z}$ scheme photocatalyst. A representative example was recently reported by Shi et al. [35], who fabricated a $\mathrm{Cu}_{2} \mathrm{~S} / \mathrm{Zn}_{0.67} \mathrm{Cd}_{0.33} \mathrm{~S}$ 2D/2D atomic-level heterojunction with a lamellar hexagonal morphology. Because of the $2 \mathrm{D} / 2 \mathrm{D}$ atomic-level compact interface and efficient $\mathrm{Z}$ - 
scheme electron-hole separation and transport (Fig. 11d), the $\mathrm{Cu}_{2} \mathrm{~S} / \mathrm{Zn}_{0.67} \mathrm{Cd}_{0.33} \mathrm{~S}$ photocatalyst displayed a remarkable photocatalytic hydrogen production activity $\left(15.27 \mathrm{mmol} \mathrm{h}^{-1} \mathrm{~g}^{-1}\right)$. More recently, BP, a new layered 2D material, was also chosen to combine with other $2 \mathrm{D}$ materials to form the 2D/2D Z-scheme photocatalyst. $\mathrm{Hu}$ et al. [153] reported the assembling of $2 \mathrm{D}$ monolayer $\mathrm{Bi}_{2} \mathrm{WO}_{6}(\mathrm{MBWO})$ with $2 \mathrm{D}$ layered $\mathrm{BP}$ to form a novel Zscheme 2D/2D heterojunction (Fig. 11e). The $\mathrm{H}_{2}$ evolution rate of $\mathrm{BP} / \mathrm{MBWO}$ can reach $21,042 \mu \mathrm{mol} \mathrm{g}^{-1}$, which is 9.15 times that of pristine MBWO.

More recently, a new step-scheme (S-scheme) heterojunction concept was proposed based on the Z-scheme photocatalysts [116,154-158]. As shown in Fig. 9d, two ntype semiconductor photocatalysts were coupled together to form the S-scheme heterojunction, in which one acts as an oxidation photocatalyst (Semiconductor A) and the other is reduction photocatalyst (Semiconductor B). Due to the driving force of the internal electric field in Sscheme heterojunction, the excited electrons in the CB of oxidation photocatalysts will easily recombine with holes in the VB of the reduction photocatalysts. Significantly, the strongly oxidative holes in the VB of oxidation pho- tocatalysts and the strongly reductive electrons in the $\mathrm{CB}$ of reduction photocatalysts will be spatially separated. The S-scheme strategy can not only efficiently restrain the recombination of photogenerated charges but also promote the reducing capacity and oxidizing ability of the heterojunction in the photocatalysis process. A recent study reported the design of a $2 \mathrm{D} / 2 \mathrm{D} \quad \mathrm{WO}_{3} / g-\mathrm{C}_{3} \mathrm{~N}_{4} \mathrm{~S}$ scheme heterojunction for photocatalytic $\mathrm{H}_{2}$ production [116], in which $2 \mathrm{D} \mathrm{g}-\mathrm{C}_{3} \mathrm{~N}_{4}$ served as a reduction-type photocatalyst while $2 \mathrm{D} \quad \mathrm{WO}_{3}$ was an oxidation-type photocatalyst. As shown in Fig. 12a and b, there is a difference of work function between $\mathrm{WO}_{3}$ and $\mathrm{g}-\mathrm{C}_{3} \mathrm{~N}_{4}$, which indicates the presence of charge transfer at the interface of $\mathrm{WO}_{3}$ and $\mathrm{g}-\mathrm{C}_{3} \mathrm{~N}_{4}$. According to the S-scheme charge transfer mechanism, the relatively useless electrons in the $\mathrm{CB}$ of $\mathrm{WO}_{3}$ and holes in the $\mathrm{VB}$ of $\mathrm{g}_{-} \mathrm{C}_{3} \mathrm{~N}_{4}$ would recombine together, whereas the useful electrons and holes in the $\mathrm{CB}$ of $\mathrm{g}-\mathrm{C}_{3} \mathrm{~N}_{4}$ and $\mathrm{VB}$ of $\mathrm{WO}_{3}$ would be respectively left to participate in photocatalytic reactions (Fig. 12c-e). As a result, the electrons left in the in the $\mathrm{CB}$ of $\mathrm{g}^{-} \mathrm{C}_{3} \mathrm{~N}_{4}$ exhibited supreme redox capacity, thus providing a strong driving force for running the photocatalytic water splitting reaction. In a more recent study,

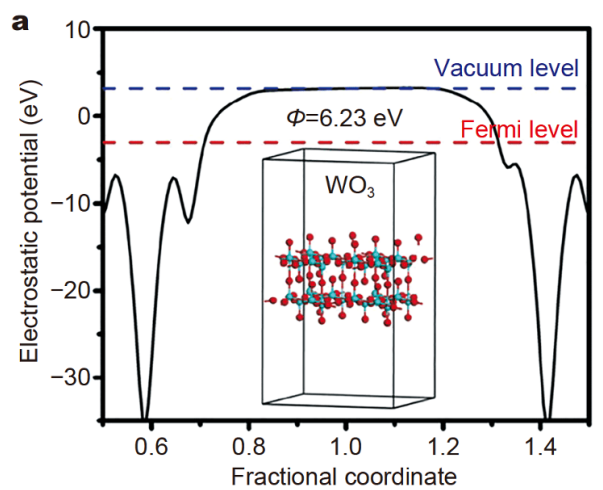

C

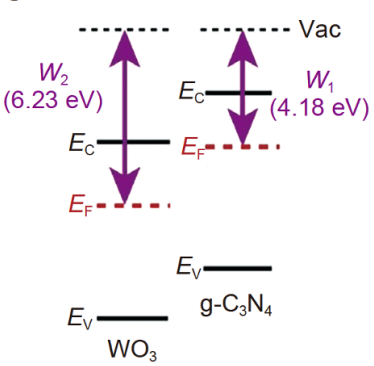

Before contact

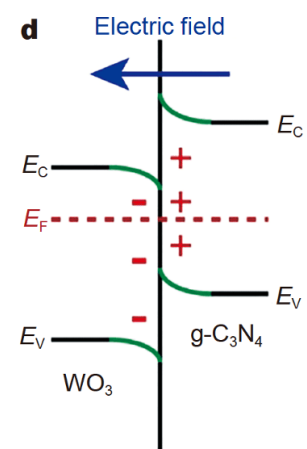

After contact
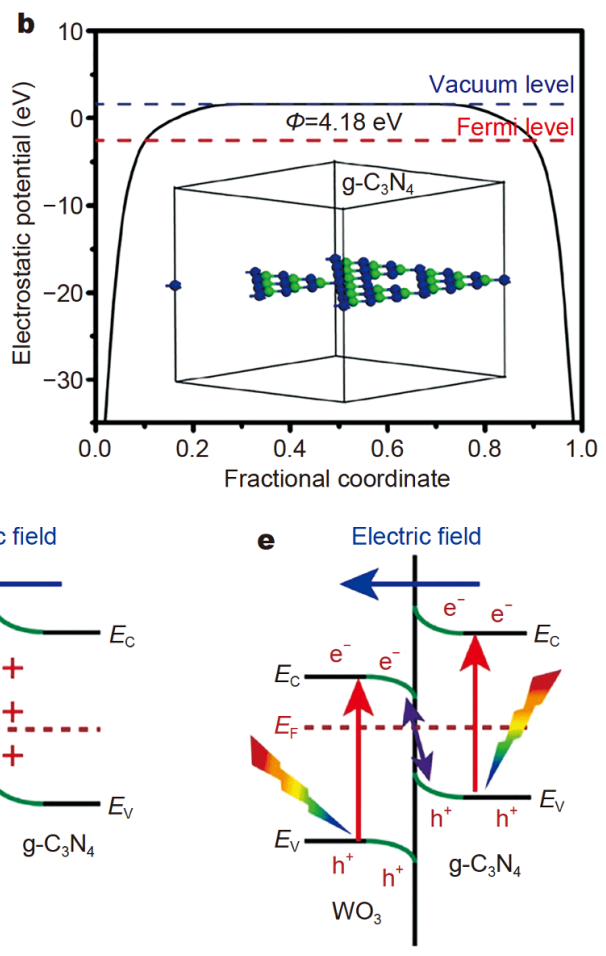

Light irradiation

Figure 12 (a) Electrostatic potentials of (a) $\mathrm{WO}_{3}(001)$ surface and (b) $g-\mathrm{C}_{3} \mathrm{~N}_{4}(001)$ surface. (c-e) S-scheme charge transfer mechanism between $\mathrm{WO}_{3}$ and $g-C_{3} \mathrm{~N}_{4}$. Reprinted with permission from Ref. [116]. Copyright 2019, Elsevier. 
Ren et al. [159] employed 2D CdS to combine with 2D g$\mathrm{C}_{3} \mathrm{~N}_{4}$ to form an S-scheme heterojunction for photocatalytic $\mathrm{H}_{2}$ production. They also demonstrated that the formation of the $2 \mathrm{D} / 2 \mathrm{D}$ S-scheme heterojunction could accelerate the interfacial charge separation for surface reaction. The $\mathrm{CdS} / \mathrm{g}-\mathrm{C}_{3} \mathrm{~N}_{4} 2 \mathrm{D} / 2 \mathrm{D}$ S-scheme heterojunction showed a high $\mathrm{H}_{2}$ production rate of $153,000 \mu \mathrm{mol} \mathrm{g}^{-1} \mathrm{~h}^{-1}$, which was 3.83 times and 3060 times higher than those of pure $\mathrm{CdS}$ and $\mathrm{g}-\mathrm{C}_{3} \mathrm{~N}_{4}$, respectively.

Another type of 2D/2D heterojunction used for hydrogen generation was formed by coupling $2 \mathrm{D}$ cocatalyst with 2D semiconductor photocatalysts (Fig. 9e). It has been demonstrated that suitable cocatalysts play pivotal roles in improving both the activity and reliability of semiconductor photocatalysts. The cocatalysts can not only decrease the overpotential for hydrogen production but also boost the electron-hole separation at the cocatalyst/semiconductor interface. Previously, some 0D noble metal nanoparticles were often employed as efficient cocatalysts. However, the small contact area between the $0 \mathrm{D}$ noble metal and 2D semiconductor severely hinders the charge transfer at the interface. Moreover, the high cost and scarcity of noble metals limit their practical applications. In recent years, various earth-abundant 2D noble-metal-free cocatalysts have been emerging as potential candidates to replace noble metals. For example, $\mathrm{MoS}_{2}$, a rising star in 2D layered materials, has been reported as an efficient cocatalyst on a variety of $2 \mathrm{D}$ semiconductor photocatalysts. Yuan and coworkers [40] loaded 2D MoS 2 nanosheets on the surface of 2D anatase $\mathrm{TiO}_{2}$ nanosheets with exposed (001) facets. The photocatalytic $\mathrm{H}_{2}$-production activity of $\mathrm{TiO}_{2}$ was significantly improved by loading an optimal amount of $0.50 \mathrm{wt} \%$ $\mathrm{MoS}_{2}$ as a cocatalyst. Later, the authors also used 2D $\mathrm{MoS}_{2}$ nanosheets cocatalyst to modify $\mathrm{Cu}^{2+}$-doped $\mathrm{ZnIn}_{2} \mathrm{~S}_{4}\left(\mathrm{Cu}-\mathrm{ZnIn}_{2} \mathrm{~S}_{4}\right)$ nanosheets for highly efficient solar-to- $\mathrm{H}_{2}$ conversion [160]. It was found that the $2 \mathrm{D} / 2 \mathrm{D}$ $\mathrm{MoS}_{2} / \mathrm{Cu}-\mathrm{ZnIn}_{2} \mathrm{~S}_{4}$ photocatalyst at a $6 \mathrm{wt} \% \mathrm{MoS}_{2}$ loading amount achieved a higher $\mathrm{H}_{2}$-evolution rate of $5,463 \mu \mathrm{mol} \mathrm{g}^{-1} \mathrm{~h}^{-1}$. Ma et al. [86] demonstrated that the photocatalytic $\mathrm{H}_{2}$-evolution activity of $2 \mathrm{D} \mathrm{CdS}$ nanosheets could be significantly boosted by loading ultrathin $\mathrm{MoS}_{2}$ nanosheets. The photocatalytic $\mathrm{H}_{2}$ evolution performance of $2 \mathrm{D}$ layered niobate oxides (e.g., $\mathrm{SnNb}_{2} \mathrm{O}_{6}$ ) can also be improved by coupling $2 \mathrm{D} \mathrm{MoS}_{2}$ nanosheets [70]. More recently, Yuan's group [82] further employed 2D $\mathrm{MoS}_{2}$ nanosheets to combine with 2D metal-free semiconductors to form $\mathrm{BP} / \mathrm{MoS}_{2}$ and $\mathrm{g}-\mathrm{C}_{3} \mathrm{~N}_{4} / \mathrm{MoS}_{2}$ [81] $2 \mathrm{D} / 2 \mathrm{D}$ composites. In the $\mathrm{BP} / \mathrm{MoS}_{2} 2 \mathrm{D} / 2 \mathrm{D}$ photocatalyst system, the $\mathrm{MoS}_{2}$ acts as both an electron sink and a cocatalyst which will greatly reduce the recombination of electron-hole pairs, while the 2D/2D smart structure provides large $2 \mathrm{D}$ nanointerfaces for photogenerated charge transfer, and thus synergistically promotes the $\mathrm{H}_{2}$ evolution rate (Fig. 13a). As a result, the $\mathrm{BP} / \mathrm{MoS}_{2} 2 \mathrm{D} / 2 \mathrm{D}$ photocatalyst loaded with $10 \% \mathrm{MoS}_{2}$ showed a $\mathrm{H}_{2}$ evolution rate of $1,286 \mu \mathrm{mol} \mathrm{g}^{-1} \mathrm{~h}^{-1}$, which is higher than that of other counterparts (Fig. 13b). Besides $\mathrm{MoS}_{2}$, MXene, a new family of 2D material, possesses excellent electrical conductivity, good hydrophilicity, and lower Fermi level compared with semiconductors, which could also serve as an effective cocatalyst for photocatalytic hydrogen production from water. Su et al. [119] synthesized 2D/2D $\mathrm{Ti}_{3} \mathrm{C}_{2} / \mathrm{g}-\mathrm{C}_{3} \mathrm{~N}_{4}$ composites as photocatalysts for hydrogen evolution under visible light irradiation. As shown in Fig. $13 \mathrm{c}$, a Schottky barrier was present at the $\mathrm{Ti}_{3} \mathrm{C}_{2} / \mathrm{g}$ $\mathrm{C}_{3} \mathrm{~N}_{4}$ interface when $\mathrm{Ti}_{3} \mathrm{C}_{2}$ was coupled with $\mathrm{g}-\mathrm{C}_{3} \mathrm{~N}_{4}$, which can serve as the electron reservoir, thus prompting the separation of photoinduced electrons and holes. In addition, the large intimate $2 \mathrm{D} / 2 \mathrm{D}$ interface between the g- $\mathrm{C}_{3} \mathrm{~N}_{4}$ and $\mathrm{Ti}_{3} \mathrm{C}_{2}$ can shorten the charge transfer distance, leading to the greatly improved migration rate of the photoinduced electrons. The results revealed that the 2D/2D $\mathrm{Ti}_{3} \mathrm{C}_{2} / \mathrm{g}-\mathrm{C}_{3} \mathrm{~N}_{4}$ composites displayed a 10 times higher photocatalytic hydrogen evolution activity than that of pristine $\mathrm{g}-\mathrm{C}_{3} \mathrm{~N}_{4}$ (Fig. 13d). Similarly, Lin et al. [118] fabricated a $2 \mathrm{D} / 2 \mathrm{D} \mathrm{Ti}_{3} \mathrm{C}_{2}$ MXene/O-doped g- $\mathrm{C}_{3} \mathrm{~N}_{4}$ Schottky-junction photocatalyst, which exhibited a high hydrogen evolution rate of $25,124 \mu \mathrm{mol} \mathrm{g}^{-1} \mathrm{~h}^{-1}$.

Multicomponent 2D cocatalysts have also been investigated to improve the photocatalytic hydrogen production performance of semiconductor photocatalysts. Li et al. [75] reported that $2 \mathrm{D} \mathrm{TiO}_{2}$ with co-exposed (101) and (001) facets was modified by $\mathrm{Ti}_{3} \mathrm{C}_{2}$ and $\mathrm{MoS}_{2}$ bicomponent cocatalysts toward enhanced photocatalytic hydrogen production activity. In this ternary $2 \mathrm{D} / 2 \mathrm{D} / 2 \mathrm{D}$ $\mathrm{Ti}_{3} \mathrm{C}_{2} @ \mathrm{TiO}_{2} @ \mathrm{MoS}_{2}$, the photoexcited electrons can migrate from the (001) facets of $\mathrm{TiO}_{2}$ to (101) facets and $\mathrm{Ti}_{3} \mathrm{C}_{2}$, respectively, and then the electrons on the (101) facets transport to $\mathrm{MoS}_{2}$, leading to a dual-carrier-separation manner (Fig. 13e). Therefore, an electron-rich environment was acquired on the planar surfaces of $\mathrm{Ti}_{3} \mathrm{C}_{2}$ and $\mathrm{MoS}_{2}$, on which the $\mathrm{H}_{2} \mathrm{O}$ was reduced to produce $\mathrm{H}_{2}$ with a higher rate of $6,425.297 \mu \mathrm{mol} \mathrm{h}^{-1} \mathrm{~g}^{-1}$ (Fig. 13f). In some other studies, other $2 \mathrm{D}$ sulfide-based materials as well metal phosphides were also employed as cocatalysts to modify some $2 \mathrm{D}$ semiconductor photocatalysts for boosting the performance of photocatalytic hydrogen evolution, such as $\mathrm{Ni}_{2} \mathrm{P} / \mathrm{ZnIn}_{2} \mathrm{~S}_{4}$ [110] and $\mathrm{SnS}_{2} / \mathrm{TiO}_{2}$ [78]. 

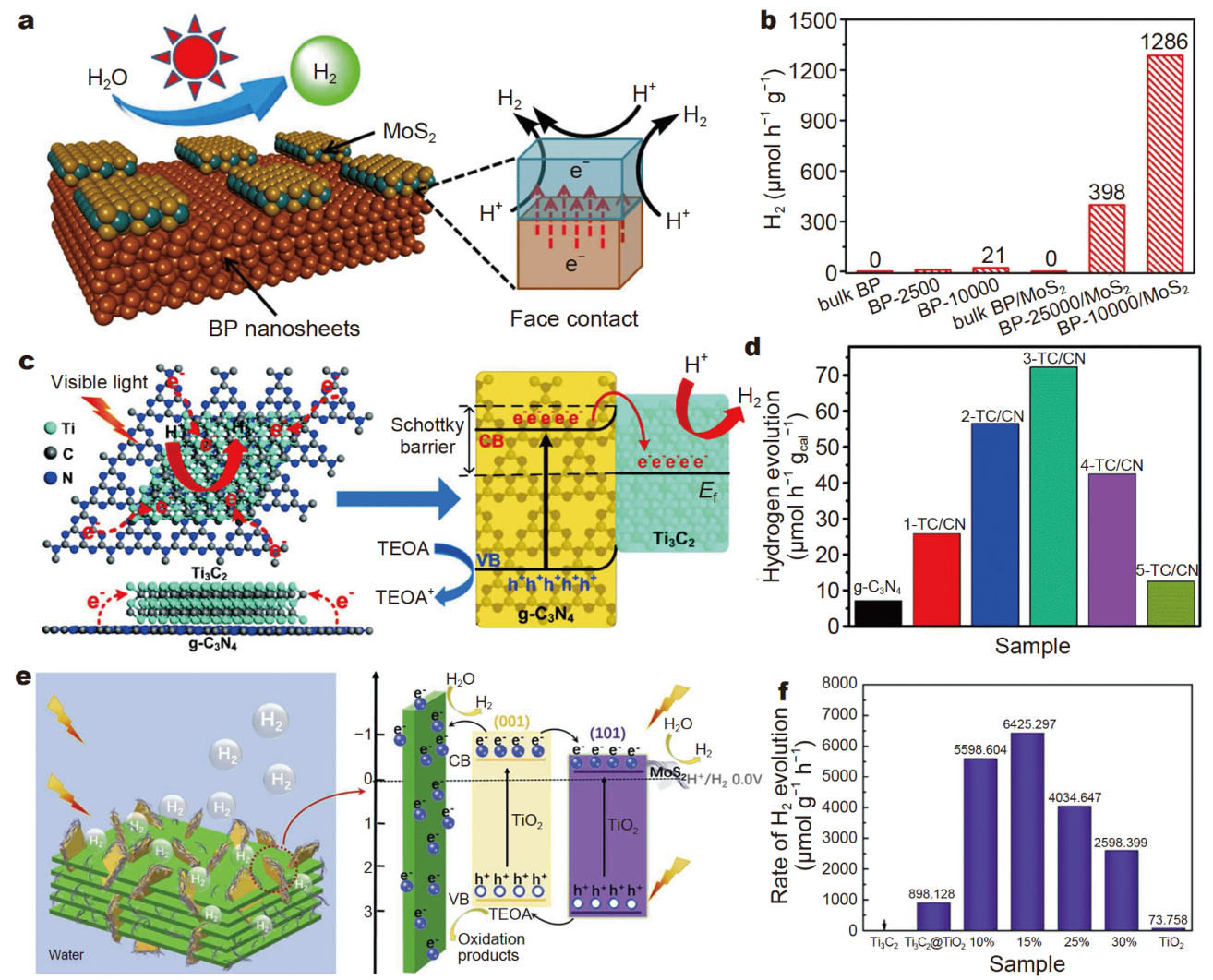

Figure 13 Schematic diagrams of the composite photocatalysts and the corresponding charge transfer route, and comparison of the $\mathrm{H}_{2}$ production rates over the composite photocatalysts with their respective counterparts. (a, b) 2D/2D BP/MoS ${ }_{2}$. Reprinted with permission from Ref. [82]. Copyright 2019, Elsevier. (c, d) $2 \mathrm{D} / 2 \mathrm{D} \mathrm{Ti} \mathrm{C}_{3} / \mathrm{g}-\mathrm{C}_{3} \mathrm{~N}_{4}$. Reprinted with permission from Ref. [119]. Copyright 2019, Elsevier. (e, f) 2D/2D/2D $\mathrm{Ti}_{3} \mathrm{C}_{2} @ \mathrm{TiO}_{2} @ \mathrm{MoS}_{2}$. Reprinted with permission from Ref. [75]. Copyright 2019, Elsevier.

\section{$\mathrm{CO}_{2}$ reduction}

Nowadays, the continuous consumption of fossil fuel for industrial manufacturing has caused a terrible growing amount of $\mathrm{CO}_{2}$ in the atmosphere [159]. It has been reported that the annual anthropogenic $\mathrm{CO}_{2}$ emissions from fossil fuel combustion have reached at about $9 \mathrm{Gt}$ $\left(1 \mathrm{G}=10^{9}\right)$, which is approximately $43 \%$ higher than the level recorded in pre-industrial times [161,162]. The substantial rise of $\mathrm{CO}_{2}$ in atmosphere would seriously damage the balance of the earth's carbon cycle and trigger global warming. Thus, many strategies have been explored to reduce the $\mathrm{CO}_{2}$ concentration in atmosphere, such as electrochemical [163], biological [164], thermochemical [165] and photocatalytic means [166,167]. Among them, photocatalytically converting $\mathrm{CO}_{2}$ into valuable energy fuels (e.g., $\mathrm{CH}_{4}, \mathrm{CH}_{3} \mathrm{OH}, \mathrm{CO}$ ) by utilizing solar energy has been regarded as a "kill two birds with one stone" ideal approach in terms of protecting our environment and simultaneously supplying energy $[161,168]$. Since the first discovery of photoconversion of
$\mathrm{CO}_{2}$ to valuable fuels over semiconductor materials by Inoue and coworkers [169] in 1979, numerous studies have been conducted on the preparation of highly efficient photocatalysts to meet the requirements of practical application of $\mathrm{CO}_{2}$ photoreduction [131,170-176]. As mentioned above, various products can be derived from photocatalytic $\mathrm{CO}_{2}$ reduction via multi-step reaction pathways. The following equations give the half reactions to the various products commonly formed in $\mathrm{CO}_{2}$ reduction [161]. In addition, the theoretical reduction potential $\left(E^{0}\right)(\mathrm{V}, v s$. NHE at $\mathrm{pH} 7)$ for each half equation is also provided.

$$
\begin{aligned}
& \mathrm{CO}_{2}+\mathrm{e}^{-} \rightarrow \cdot \mathrm{CO}_{2}^{-}, E^{0}=-1.90 \mathrm{~V} \\
& \mathrm{CO}_{2}+2 \mathrm{e}^{-}+2 \mathrm{H}^{+} \rightarrow \mathrm{HCOOH}, E^{0}=-0.61 \mathrm{~V} \\
& \mathrm{CO}_{2}+2 \mathrm{e}^{-}+2 \mathrm{H}^{+} \rightarrow \mathrm{CO}+\mathrm{H}_{2} \mathrm{O}, E^{0}=-0.53 \mathrm{~V} \\
& \mathrm{CO}_{2}+4 \mathrm{e}^{-}+4 \mathrm{H}^{+} \rightarrow \mathrm{HCHO}+\mathrm{H}_{2} \mathrm{O}, E^{0}=-0.48 \mathrm{~V}
\end{aligned}
$$




$$
\begin{aligned}
& \mathrm{CO}_{2}+6 \mathrm{e}^{-}+6 \mathrm{H}^{+} \rightarrow \mathrm{CH}_{3} \mathrm{OH}+\mathrm{H}_{2} \mathrm{O}, E^{0}=-0.38 \mathrm{~V} ; \\
& \mathrm{CO}_{2}+8 \mathrm{e}^{-}+8 \mathrm{H}^{+} \rightarrow \mathrm{CH}_{4}+2 \mathrm{H}_{2} \mathrm{O}, E^{0}=-0.28 \mathrm{~V} .
\end{aligned}
$$

Clearly, the final products depend upon the thermodynamical mechanism to conduct the reaction, such as the number of electrons participating in the reaction, electron migration rate, and $\mathrm{CB}$ and VB potentials of the photocatalysts. Thus, the studies in this area need not only to obtain good efficiency but also to increase the selectivity for specific products. Particularly, the utilization of $2 \mathrm{D} / 2 \mathrm{D}$ heterojunction nanomaterials in the field of $\mathrm{CO}_{2}$ photoreduction has fascinated many researchers (Table 3). Combining different 2D materials can establish a strong interface contact as well as large contact surface to allow the rapid transfer and separation of photoinduced charge carriers across the heterojunction interface, thus leading to greatly improved photocatalytic $\mathrm{CO}_{2}$ reduction efficiency and selectivity. Ong et al. [96] reported the incorporation of $\mathrm{rGO}$ with protonated $\mathrm{g}_{-} \mathrm{C}_{3} \mathrm{~N}_{4}$ (pCN) to form an rGO-pCN 2D/2D hybrid heterojunction towards photocatalytic reduction of $\mathrm{CO}_{2}$ to $\mathrm{CH}_{4}$. It has been confirmed that rGO has excellent charge mobility and high electron storage capacity, which often acts as cocatalyst to provide conductive electron channels for the separation of photogenerated charges in the heterojunction photocatalyst. In this work, the photogenerated electrons in the $\mathrm{CB}$ of $\mathrm{pCN}$ would easily migrate to $\mathrm{rGO}$ framework owing to the unique properties of $\mathrm{rGO}$ as well as the large interface contact area between $\mathrm{pCN}$ and $\mathrm{rGO}$. Then, the photoinduced holes left in the VB of pCN will react with $\mathrm{H}_{2} \mathrm{O}$ molecules to produce protons $\left(\mathrm{H}^{+}\right)$, while the electrons on the rGO would not only interact with the $\mathrm{H}^{+}$to generate $\cdot \mathrm{H}$ radicals but also react with the activated $\mathrm{CO}_{2}$ molecules to form superoxide $\left(\cdot \mathrm{CO}_{2}{ }^{-}\right)$radicals. Subsequently, the $\mathrm{CH}_{4}$ product was obtained through a series of radical reactions between $\cdot \mathrm{CO}_{2}^{-}$and $\cdot \mathrm{H}$. The results revealed that the total amount of $\mathrm{CH}_{4}$ production was 5.4-folds higher than that of pure pCN photocatalysts. $2 \mathrm{D}$ rGO cocatalyst was also reported to combine with $\mathrm{SiC}$ nanosheets by $\mathrm{Han}$ and coworkers [33] for $\mathrm{CO}_{2}$ photoreduction with high efficiency and $\mathrm{CH}_{4}$ selectivity. In this photoreduction system, the robust $2 \mathrm{D} / 2 \mathrm{D} \mathrm{SiC/}$ rGO heterojunction allowed fast transfer of energetic electrons from $\mathrm{SiC}$ to $\mathrm{rGO}$ and the proportion of rGO had important effects on both the activity and selectivity. The results demonstrated that a low proportion of rGO in the composite would accumulate dense energetic electrons (Fig. 14a and b), promoting the eight-electron process for $\mathrm{CH}_{4}$ generation, whereas a high proportion of

rGO would result in sparse energetic electrons on rGO, paving the two-electron process for $\mathrm{CO}$ generation. In another study, Cao et al. [28] employed ultrathin $\mathrm{Ti}_{3} \mathrm{C}_{2}$ nanosheets as a cocatalyst to couple with $\mathrm{Bi}_{2} \mathrm{WO}_{6}$ nanosheets for photocatalytic $\mathrm{CO}_{2}$ reduction. Benefiting from the unique 2D/2D heterojunction, the $\mathrm{CO}_{2}$ adsorption capability was enhanced and the photoinduced electrons could quickly transfer from $\mathrm{B}_{\mathrm{i} 2} \mathrm{WO}_{6}$ to the surface of $\mathrm{Ti}_{3} \mathrm{C}_{2}$ (Fig. 14c). As a result, the $2 \mathrm{D} / 2 \mathrm{D} \mathrm{Ti}_{3} \mathrm{C}_{2} /$ $\mathrm{B}_{\mathrm{i} 2} \mathrm{WO}_{6}$ photocatalyst exhibited 4 and 6 times higher productive rates of $\mathrm{CH}_{4}$ and $\mathrm{CH}_{3} \mathrm{OH}$ than that of pristine $\mathrm{Bi}_{2} \mathrm{WO}_{6}$ nanosheets, respectively.

2D/2D Z-scheme photocatalysts have also been reported for enhanced photoreduction $\mathrm{CO}_{2}$ activity. Wang et al. [47] synthesized Z-scheme $\mathrm{BiOI} / \mathrm{g}-\mathrm{C}_{3} \mathrm{~N}_{4} 2 \mathrm{D} / 2 \mathrm{D}$ photocatalyst for the reduction of $\mathrm{CO}_{2}$ to produce $\mathrm{CO}, \mathrm{H}_{2}$ and/or $\mathrm{CH}_{4}$. Under visible light irradiation, the synthesized photocatalyst exhibited more highly efficient photoreduction $\mathrm{CO}_{2}$ activity than pure $\mathrm{g}-\mathrm{C}_{3} \mathrm{~N}_{4}$ and $\mathrm{BiOI}$ along. Another example of $2 \mathrm{D} / 2 \mathrm{D}$ hybrid $\mathrm{Z}$-scheme heterojunction used for photoreduction of $\mathrm{CO}_{2}$ was reported by Jo and coworkers [50]. In this work, they constructed a $\mathrm{Bi}_{2} \mathrm{WO}_{6} / \mathrm{rGO} / \mathrm{g}-\mathrm{C}_{3} \mathrm{~N}_{4}$ hybrid heterojunction, in which the $\mathrm{rGO}$ acted as not only a supporter to capture the electrons from $\mathrm{g}-\mathrm{C}_{3} \mathrm{~N}_{4}$, but also the redox mediator to promote the $\mathrm{Z}$-scheme charge transfer be-

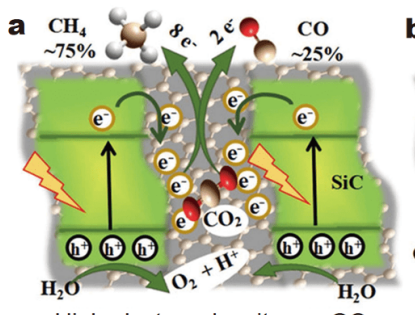

High electron density on $\mathrm{rGO}$
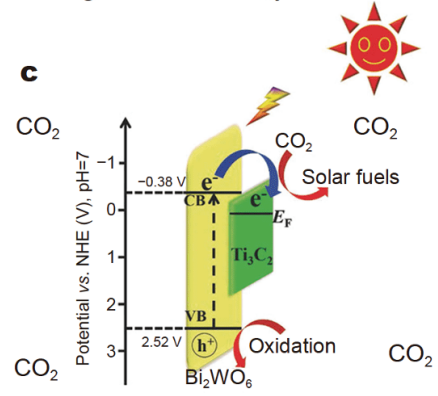

Figure 14 (a, b) Schematic diagrams of high (a) and low (b) electron density-dependent $\mathrm{CH}_{4}$ selectivity in SiC/rGO heterojunctions. Reprinted with permission from Ref. [33]. Copyright 2018, Wiley-VCH. (c) Energy level structure diagram of $\mathrm{Bi}_{2} \mathrm{WO}_{6}$ and $\mathrm{Ti}_{3} \mathrm{C}_{2}$, as well as the photoinduced electron transfer process. Reprinted with permission from Ref. [28]. Copyright 2018, Wiley-VCH. 
tween $\mathrm{g}_{-} \mathrm{C}_{3} \mathrm{~N}_{4}$ and $\mathrm{Bi}_{2} \mathrm{WO}_{6}$ (Fig. 15b). Profiting from the effects of $\mathrm{Z}$-scheme charge transfer route, large interfacial contact of the $2 \mathrm{D} / 2 \mathrm{D}$ architecture and the advantages of rGO, the $\mathrm{Bi}_{2} \mathrm{WO}_{6} / \mathrm{rGO} / \mathrm{g}-\mathrm{C}_{3} \mathrm{~N}_{4}$ hybrid photocatalyst showed a higher yield of carbonaceous products ( $\mathrm{CO}$ $\left.+\mathrm{CH}_{4}\right)$ as well as enhanced selectivity of the products (Fig. 15a).

The 2D/2D heterojunction photocatalysts with a TypeII configuration have also been applied in photocatalytic $\mathrm{CO}_{2}$ reduction. Tonda et al. [57] constructed a $2 \mathrm{D} / 2 \mathrm{D}$ Type-II heterojunction between the negatively charged
$2 \mathrm{D} \mathrm{g}-\mathrm{C}_{3} \mathrm{~N}_{4}$ nanosheets and positively charged NiAl-LDH for photocatalytic $\mathrm{CO}_{2}$ reduction. Because of the synergistic effect of $2 \mathrm{D} / 2 \mathrm{D}$ strong interfacial contact and efficient charge carrier transfer mode of Type-II, the resulting 2D/2D g- $\mathrm{C}_{3} \mathrm{~N}_{4} / \mathrm{NiAl}-\mathrm{LDH}$ showed 5- and 9-fold enhancement of $\mathrm{CO}$ production rate than pure $\mathrm{g}-\mathrm{C}_{3} \mathrm{~N}_{4}$ and NiAl-LDH, respectively. More recently, Kong and coworkers [125] developed 2D/2D surface defect-engineered $\mathrm{Bi}_{2} \mathrm{WO}_{6} / \mathrm{BiOI} \mathrm{p}-\mathrm{n}$ heterojunction photocatalysts (BWO-OV/BOI) towards superior photocatalytic $\mathrm{CO}_{2}$ reduction activity. As show in Fig. $15 \mathrm{c}$, the authors

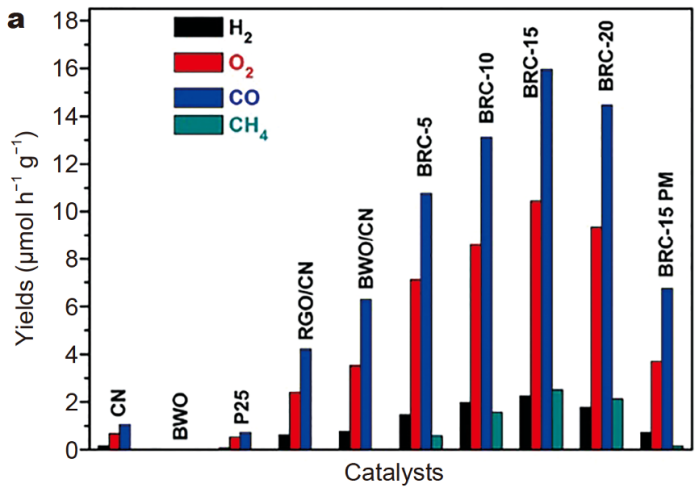

C

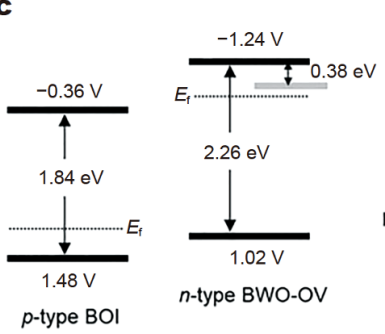

Before contact

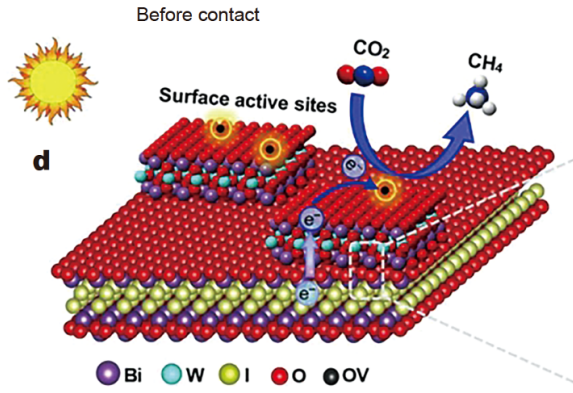

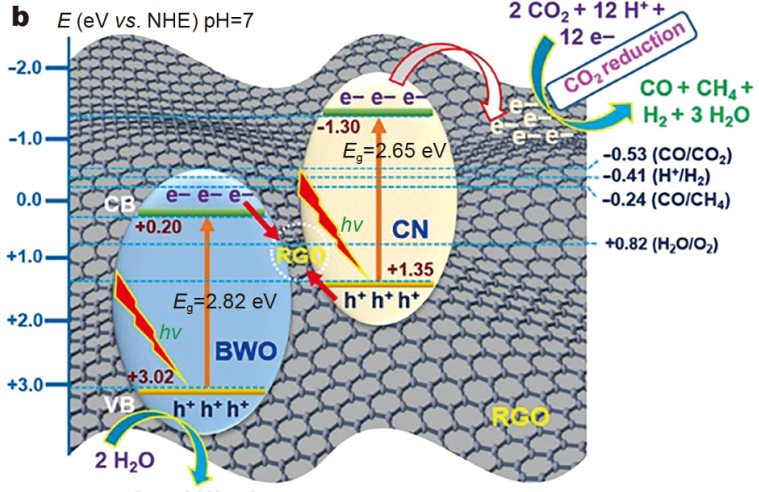

$\mathrm{O}_{2}+4 \mathrm{H}^{+}+4 \mathrm{e}-$

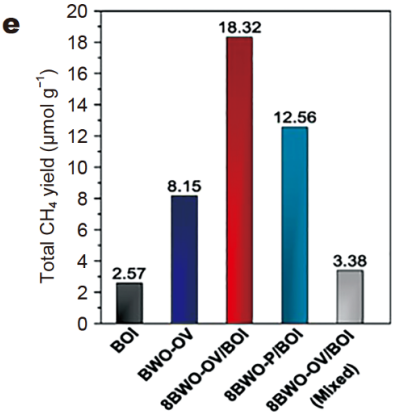

After contact
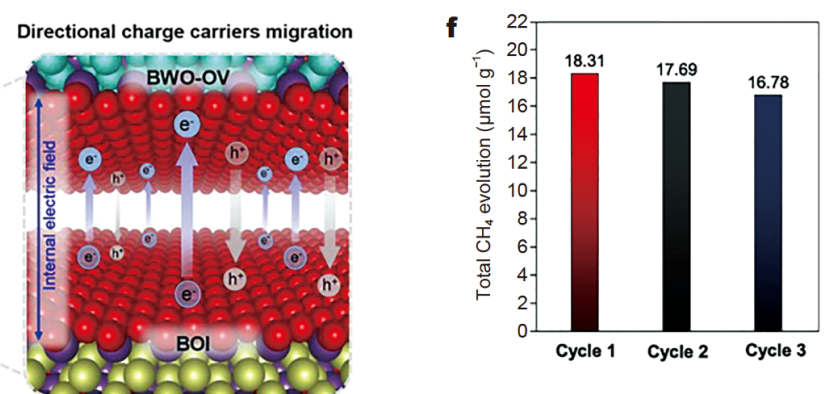

Figure 15 (a) Comparison of the photocatalytic $\mathrm{CO}, \mathrm{CH}_{4}, \mathrm{H}_{2}$, and $\mathrm{O}_{2}$ production rates over the $\mathrm{Bi}_{2} \mathrm{WO}_{6} / \mathrm{rGO} / \mathrm{g}-\mathrm{C}_{3} \mathrm{~N}_{4}$ samples as well as other synthesized photocatalysts. (b) Schematic illustration of the proposed mechanism for $\mathrm{CO}_{2}$ photoreduction in the $\mathrm{Bi}_{2} \mathrm{WO} \mathrm{O}_{6} / \mathrm{rGO} \mathrm{g}-\mathrm{C}_{3} \mathrm{~N}_{4}$ sample. Reprinted with permission from Ref. [50]. Copyright 2018, Elsevier. (c) Estimated relative band positions and schematic diagram of charge transfer and separation at the BWO-OV/BOI composite. (d) Schematic illustration for the proposed mechanism of photocatalytic $\mathrm{CO}_{2}$ reduction over the BWO-OV/BOI composite. (e) Total yield of $\mathrm{CH}_{4}$ production over the BWO-OV/BOI composite and other as-developed samples. (f) Photostability tests of BWO-OV/BOI composite. Reprinted with permission from Ref. [125]. Copyright 2019, Elsevier. 
pointed out that the migration of charge carriers in this system was through the $\mathrm{p}$-n heterojunction with the builtin internal electric field instead of the conventional TypeII heterojunction when $\mathrm{Bi}_{2} \mathrm{WO}_{6}$ and $\mathrm{BiOI}$ came into contact. What is more, the $2 \mathrm{D} / 2 \mathrm{D}$ motif allowed directional migration and spatial separation of photoinduced charge carriers to opposite sides in the heterostructure, which effectively hindered their recombination (Fig. 15d). The BWO-OV/BOI photocatalytic system displayed a remarkable visible-light-driven $\mathrm{CH}_{4}$ production yield of $18.32 \mu \mathrm{mol} \mathrm{g}^{-1}$, which is 7.1 -fold enhancement over the pure BOI photocatalysts (Fig. 15d). In addition, the $\mathrm{CH}_{4}$ production yield over the BWO-OV/BOI composite can be maintained about $92 \%$ of the initial value after three consecutive test cycles, suggesting its superior photostability (Fig. 15e).

\section{Removal of pollutions}

In the past few decades, the rapid development of urbanization and industrialization has simultaneously led to the increasing serious environmental problems, such as discharge of wastewater and spoil gas that often contain various toxic species, which do great harm to human health and ecosystem equilibrium $[177,178]$. Thus, a great number of methods, including chemical degradation [179], physical adsorption [180], and biodegradation techniques [181] have been adopted for environment remediation. However, most of the conventional treatments for environment remediation were found inefficient and may cause secondary pollution. In recent years, sunlight-driven photocatalysis technology is considered to be a green, effective and economic method to handle the removal of environmental pollutions [182187]. To date, tremendous efforts have been contributed to developing efficient photocatalysts to match the requirements of practical application [188-191]. It is well accepted that the development of heterostructured photocatalysts comprising multiple components possess efficient charge carrier separation abilities as compared with that of the single one, which is deemed as a promising strategy for achieving highly efficient photocatalytic activity towards environment remediation [192194]. In particular, the composite photocatalysts with $2 \mathrm{D} /$ $2 \mathrm{D}$ heterojunction often display more excellent separation of electron-hole pairs due to the intimate face-to-face contact, leading to the enhanced photocatalytic removal of environmental pollutions. Up to now, plentiful of 2D/ $2 \mathrm{D}$ heterojunctions have been applied for the removal of various kinds of pollutions, which are summarized in Table 3. Among many $2 \mathrm{D} / 2 \mathrm{D}$ materials, the $\mathrm{g}-\mathrm{C}_{3} \mathrm{~N}_{4}$ based heterojunctions are the most frequently studied photocatalysts applied in environmental remediation. For example, $2 \mathrm{D}$ chalcogenide was often coupled with g- $\mathrm{C}_{3} \mathrm{~N}_{4}$ to form a $2 \mathrm{D} / 2 \mathrm{D}$ heterojunction towards the elimination of environmental pollution. Zhang et al. [97] fabricated 2D/ $2 \mathrm{D} \mathrm{SnS} / 2 / \mathrm{g}_{3} \mathrm{~N}_{4}$ heterojunction nanosheets for the degradation of organic dyes and phenols. The formed TypeII heterojunction between $\mathrm{SnS}_{2}$ and $\mathrm{g}-\mathrm{C}_{3} \mathrm{~N}_{4}$ as well as the larger contact interface region of $2 \mathrm{D} / 2 \mathrm{D}$ construction greatly improved the separation of the electron-hole pairs, thus resulting in the enhanced photocatalytic activities for the degradation of pollutants as compared with pure g- $\mathrm{C}_{3} \mathrm{~N}_{4}$ and $\mathrm{SnS}_{2}$ nanosheets. More recently, Huo et al. [69] and Song et al. [123] further studied the uses of $2 \mathrm{D} / 2 \mathrm{D} \mathrm{SnS} / \mathrm{g}-\mathrm{C}_{3} \mathrm{~N}_{4}$ heterojunction for photodegradation of organic dyes, respectively. However, both of the authors of these two studies pointed out that charge migration mechanism between $\mathrm{SnS}_{2}$ and $\mathrm{g}-\mathrm{C}_{3} \mathrm{~N}_{4}$ should be a $\mathrm{Z}$-scheme mode rather than the conventional Type-II. The $\mathrm{MoS}_{2} / \mathrm{g}-\mathrm{C}_{3} \mathrm{~N}_{4} 2 \mathrm{D} / 2 \mathrm{D}$ heterojunction photocatalysts were also developed to remove environmental pollutants $[100,101]$. Some 2D ternary chalcogenide semiconductors were also used to couple with $\mathrm{g}-\mathrm{C}_{3} \mathrm{~N}_{4}$ to form $2 \mathrm{D} / 2 \mathrm{D}$ heterojunction photocatalysts for degradation of pollutants.

Guo et al. [68] developed a 2D/2D CuInS $2 / g-\mathrm{C}_{3} \mathrm{~N}_{4} \mathrm{Z}$ scheme heterojunction towards visible-light-driven photocatalytic degradation of tetracycline (TC). The results revealed that the $\mathrm{CuInS} / \mathrm{g}-\mathrm{C}_{3} \mathrm{~N}_{4}$ heterojunction showed a higher apparent degradation rate than that of pure g$\mathrm{C}_{3} \mathrm{~N}_{4}$ and $\mathrm{CuInS}_{2}$ nanosheets. Similarly, 2D $\mathrm{MnIn}_{2} \mathrm{~S}_{4}$ semiconductor was also reported to combine with g- $\mathrm{C}_{3} \mathrm{~N}_{4}$ to construct Z-scheme 2D/2D architectures for treatment of pharmaceutical wastewater [66]. In another study, Che et al. [195] employed yeast-derived carbon (YC) spheres as a charge carrier bridge to the $2 \mathrm{D} / 2 \mathrm{D} \mathrm{Cu} \mathrm{Cu}_{2} \mathrm{WS}_{4} / \mathrm{g}-\mathrm{C}_{3} \mathrm{~N}_{4}$ heterojunction towards photocatalytic reduction of hexavalent chromium $\mathrm{Cr}(\mathrm{VI})$ and decomposition of TC. The g- $\mathrm{C}_{3} \mathrm{~N}_{4} / \mathrm{YC} / \mathrm{Cu}_{2} \mathrm{WS}_{4}$ heterojunction congregated the merits of face-to-face $2 \mathrm{D} / 2 \mathrm{D}$ architectures, introducing a bridge for electron migration and Type-II charge transfer mode, which significantly inhibit the rapid recombination of charge carriers (Fig. 16a). As a result, The g- $\mathrm{C}_{3} \mathrm{~N}_{4} / \mathrm{YC} /$ $\mathrm{Cu}_{2} \mathrm{WS}_{4}$ showed enhanced photocatalytic activity for decomposing TC (Fig. 16b) and $\mathrm{Cr}(\mathrm{VI})$ reduction. Some bismuth-based semiconductors with a layered structure are also incorporated with $\mathrm{g}-\mathrm{C}_{3} \mathrm{~N}_{4}$ to get the $2 \mathrm{D} / 2 \mathrm{D}$ heterostructured composite toward degradation of contaminant. Wang et al. [196] constructed a novel atomic scale $2 \mathrm{D} / 2 \mathrm{D} \mathrm{g}-\mathrm{C}_{3} \mathrm{~N}_{4} / \mathrm{Bi}_{2} \mathrm{WO}_{6}$ heterojunction with a Type- 

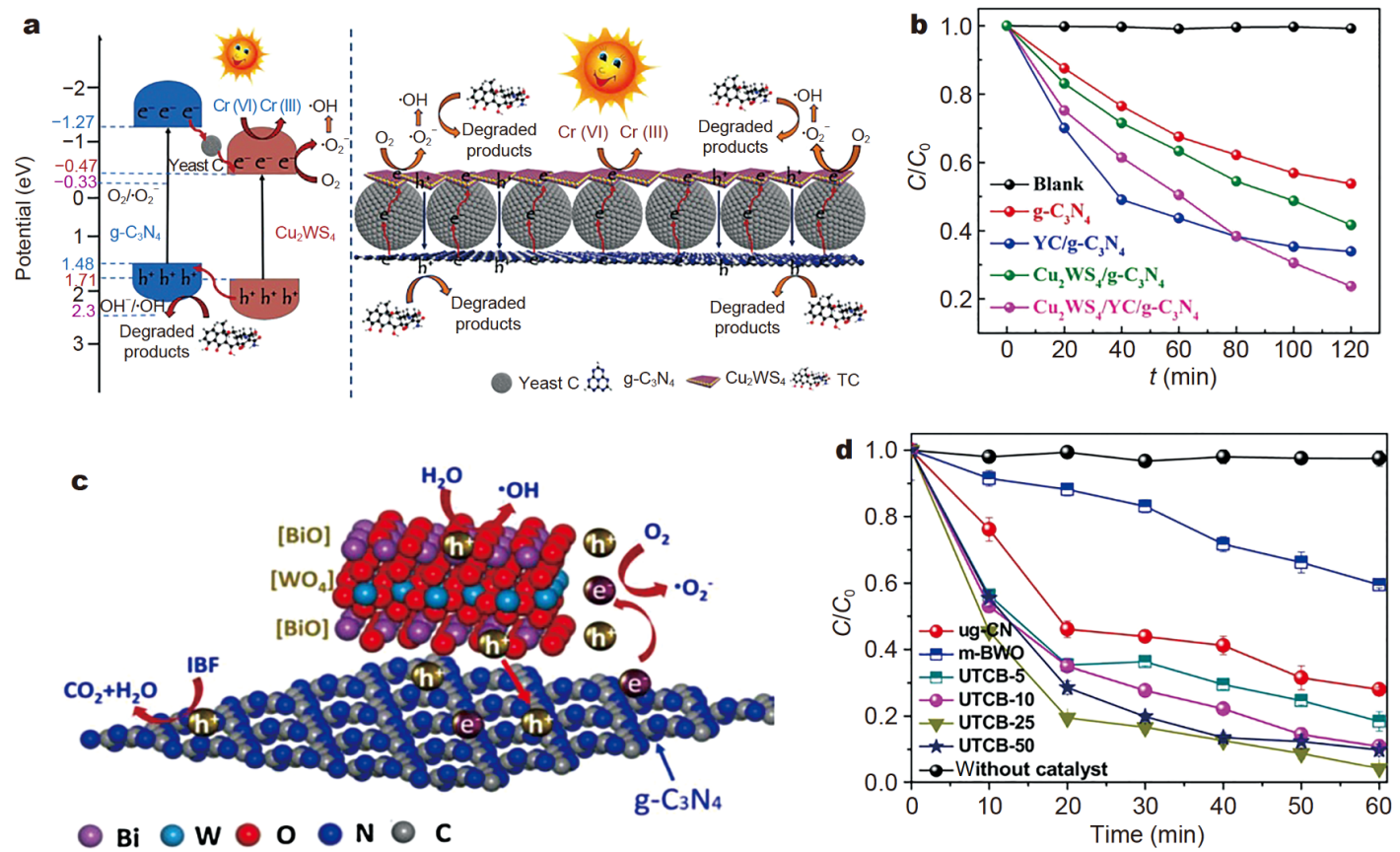

Figure 16 (a) The proposed mechanism for the enhancement of photocatalytic activity over $\mathrm{Cu}_{2} \mathrm{WS}_{4} / \mathrm{YC} / \mathrm{g}-\mathrm{C}_{3} \mathrm{~N}_{4}$ heterojunction. (b) TC degradation dynamics curves over $\mathrm{Cu}_{2} \mathrm{WS}_{4} / \mathrm{YC} / \mathrm{g}-\mathrm{C}_{3} \mathrm{~N}_{4}$ and other counterparts. Reprinted with permission from Ref. [195]. Copyright 2019, Elsevier. (c) Photocatalytic mechanism scheme of g- $\mathrm{C}_{3} \mathrm{~N}_{4} / \mathrm{Bi}_{2} \mathrm{WO}_{6} 2 \mathrm{D} / 2 \mathrm{D}$ heterojunction. (d) Photocatalytic degradation of ibuprofen by using $\mathrm{g}_{-} \mathrm{C}_{3} \mathrm{~N}_{4} / \mathrm{Bi}_{2} \mathrm{WO}_{6}$ and other samples. Reprinted with permission from Ref. [196]. Copyright 2017, Elsevier.

II band alignment (Fig. 16c), which displayed almost $96.1 \%$ ibuprofen removal efficiency of after $1 \mathrm{~h}$ visible light irradiation (Fig. 16d). Later, Guo and coworkers [111] studied that the $\mathrm{Bi}_{2} \mathrm{WO}_{6}$ and $\mathrm{g}-\mathrm{C}_{3} \mathrm{~N}_{4}$ can be constructing a Z-scheme $2 \mathrm{D} / 2 \mathrm{D}$ heterojunction to boost photoinduced charge carriers separation, leading to an enhanced visible-light-driven photodegradation efficiency. Combining g- $\mathrm{C}_{3} \mathrm{~N}_{4}$ with bismuth oxyhalides to form $2 \mathrm{D} / 2 \mathrm{D}$ heterojunction also obtained much attention in the degradation of organic pollutants. Wang et al. [58] designed an oxygen vacancy (OV)-rich ultrathin g- $\mathrm{C}_{3} \mathrm{~N}_{4}$ $\mathrm{BiOCl} 2 \mathrm{D} / 2 \mathrm{D}$ heterostructure nanosheet, which showed 95\% removal efficiency of 4-chlorophenol within $2 \mathrm{~h}$ visible light irradiation. In another study, Che and coworkers [197] employed $\mathrm{Bi}_{3} \mathrm{O}_{4} \mathrm{Cl}$ to couple with g- $\mathrm{C}_{3} \mathrm{~N}_{4}$ to assembly Z-scheme $2 \mathrm{D} / 2 \mathrm{D}$ heterojunctions. Under visible light irradiation, the $\mathrm{Z}$-scheme $\mathrm{Bi}_{3} \mathrm{O}_{4} \mathrm{Cl} / \mathrm{g}-\mathrm{C}_{3} \mathrm{~N}_{4} 2 \mathrm{D} / 2 \mathrm{D}$ heterojunctions exhibited outstanding photocatalytic activity for removing the various water contaminants. Besides these, some other types of $2 \mathrm{D}$ bismuth-contained semiconductors were also reported to couple with g- $\mathrm{C}_{3} \mathrm{~N}_{4}$ to construct $2 \mathrm{D} / 2 \mathrm{D}$ heterojunctions for environmental remediation, such as $\mathrm{C}_{3} \mathrm{~N}_{4} / \mathrm{Bi}_{20} \mathrm{TiO}_{32}$ [29], $\mathrm{BiOIO}_{3} / \mathrm{g}-\mathrm{C}_{3} \mathrm{~N}_{4}$ [109], $\mathrm{Bi}_{2} \mathrm{O}_{2} \mathrm{CO}_{3} / \mathrm{g}-\mathrm{C}_{3} \mathrm{~N}_{4}$ [99], $\mathrm{BiVO}_{4} / \mathrm{g}-\mathrm{C}_{3} \mathrm{~N}_{4}$ [120]. It can be found that the above discussed $\mathrm{g}-\mathrm{C}_{3} \mathrm{~N}_{4}$ based $2 \mathrm{D} / 2 \mathrm{D}$ photocatalysts contained at least one metal element, which would raise economic cost concern of large-scale production. Thus, the construction of $\mathrm{g}^{-} \mathrm{C}_{3} \mathrm{~N}_{4}$ based metal-free 2D/2D materials with earth-abundant elements have emerged as attractive photocatalysts for pollutant degradation in recent years. GO or rGO was often selected to combine with $\mathrm{g}_{-} \mathrm{C}_{3} \mathrm{~N}_{4}$ nanosheets to form a stable $2 \mathrm{D} / 2 \mathrm{D}$ metal-free hybrid because their excellent adsorption performance and outstanding electroconductivity. Typically, the photogenerated electrons of g- $\mathrm{C}_{3} \mathrm{~N}_{4}$ can rapidly transfer to GO or rGO via the percolation mechanism to inhibit the recombination of electron-hole pairs, and thereby leading to boosted photocatalytic activity for pollutant degradation $[98,198,199]$. Typically, the photogenerated electrons of $\mathrm{g}^{-} \mathrm{C}_{3} \mathrm{~N}_{4}$ can rapidly transfer to $\mathrm{GO}$ or $\mathrm{rGO}$ via the percolation mechanism to inhibit the recombination of electron-hole pairs, and thereby leading to boosted photocatalytic activity for pollutant degradation. Another type of metalfree $2 \mathrm{D} / 2 \mathrm{D}$ photocatalysts was composed of $\mathrm{BP}$ and $\mathrm{g}$ $\mathrm{C}_{3} \mathrm{~N}_{4}$. In the previous section, we have discussed that the $\mathrm{BP} / \mathrm{g}-\mathrm{C}_{3} \mathrm{~N}_{4} 2 \mathrm{D} / 2 \mathrm{D}$ heterojunction can be applied in photocatalytic hydrogen production. The $\mathrm{BP} / \mathrm{g}-\mathrm{C}_{3} \mathrm{~N}_{4} 2 \mathrm{D} /$ 
$2 \mathrm{D}$ photocatalysts could also be used to photodegradate organic pollutants. Wang's group [200] demonstrated that the $\mathrm{BP} / \mathrm{g}-\mathrm{C}_{3} \mathrm{~N}_{4}$ photocatalysts showed better photocatalytic activity in degradation of rhodamine $\mathrm{B}(\mathrm{RhB})$ under visible-light irradiation. More recently, Zhang et al. [124] employed the $\mathrm{BP} / \mathrm{g}-\mathrm{C}_{3} \mathrm{~N}_{4}$ photocatalysts for photocatalytic degradation of bisphenol A (BPA). The BPA degradation rate can reach to $88 \%$ over this dual-function photocatalytic system.

Bismuth-contained 2D/2D photocatalysts is another category that is often used for environmental remediation. In addition to the above discussed in coupling with g- $\mathrm{C}_{3} \mathrm{~N}_{4}$, the bismuth-based 2D layer materials can also be hybrided with other $2 \mathrm{D}$ photocatalysts to form 2D/2D heterojunction for the degradation of pollutes. Sultana et al. [107] reported a composite of $2 \mathrm{D}-\mathrm{CeO}_{2}$ with $2 \mathrm{D} \mathrm{BiOI}$ to form a Z-scheme heterojunction for $\mathrm{RhB}$ dye decolorization and phenol degradation. In another study, Xu's group [91] designed Au nanoparticles (NPs)-decorated
2D/2D $\mathrm{Bi}_{2} \mathrm{WO}_{6}-\mathrm{TiO}_{2}$ heterostructure (Fig. 17a) for photocatalytic reduction of nitroaromatics and heavy metal ions $\mathrm{Cr}(\mathrm{VI})$. In this hybrid photocatalyst system, the cooperative synergy effect (Fig. 17b), including Z-scheme as well as $2 \mathrm{D} / 2 \mathrm{D}$ rapid charge transfer platforms and surface plasmon resonance effect as well as the "electron sink" by decorated $\mathrm{Au}$ NPs, resulted in a boosted photocatalytic performance. More recently, a 2D/2D S-scheme $\operatorname{In}_{2} \mathrm{~S}_{3} /$ $\mathrm{Bi}_{2} \mathrm{O}_{2} \mathrm{CO}_{3}$ heterojunction photocatalyst was also designed for RhB degradation [156]. Benefiting from the S-scheme charge transfer mechanism as well as the face-to-face $2 \mathrm{D} /$ $2 \mathrm{D}$ structure (Fig. $17 \mathrm{c}$ ), the $2 \mathrm{D} / 2 \mathrm{D} \mathrm{In}_{2} \mathrm{~S}_{3} / \mathrm{Bi}_{2} \mathrm{O}_{2} \mathrm{CO}_{3}$ heterojunction showed 5 and 3 times $\mathrm{RhB}$ degradation capacity higher than that of pure $\mathrm{Bi}_{2} \mathrm{O}_{2} \mathrm{CO}_{3}$ and $\mathrm{In}_{2} \mathrm{~S}_{3}$ (Fig. 17d). 2D perovskite type semiconductor was also used to combine with bismuth-contained photocatalysts towards pollutant destruction. Ao et al. [45] reported the coupling perovskite type semiconductor lanthanide titanate $\left(\mathrm{La}_{2} \mathrm{Ti}_{2} \mathrm{O}_{7}\right)$ with bismuth oxybromide ( $\left.\mathrm{BiOBr}\right)$ to
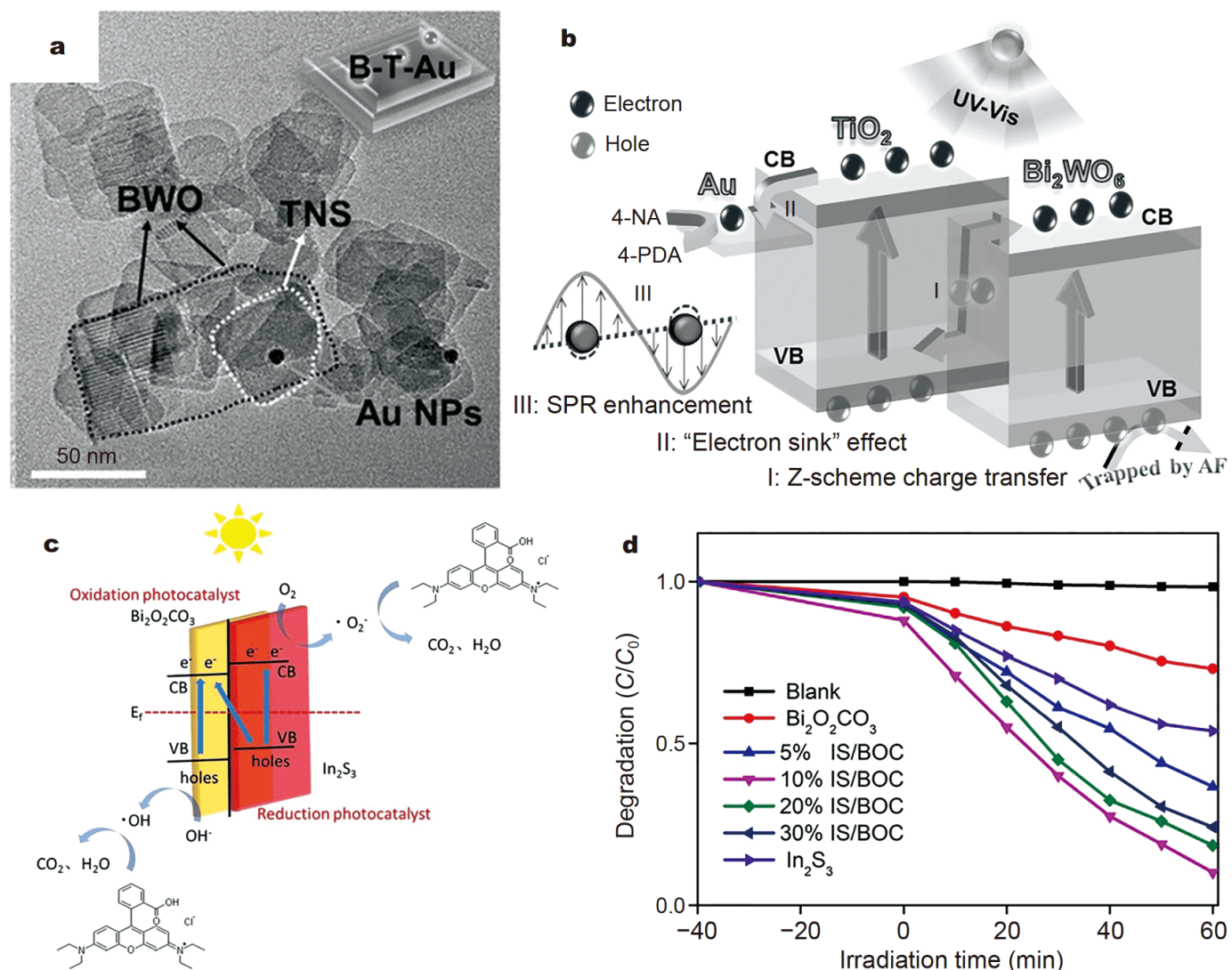

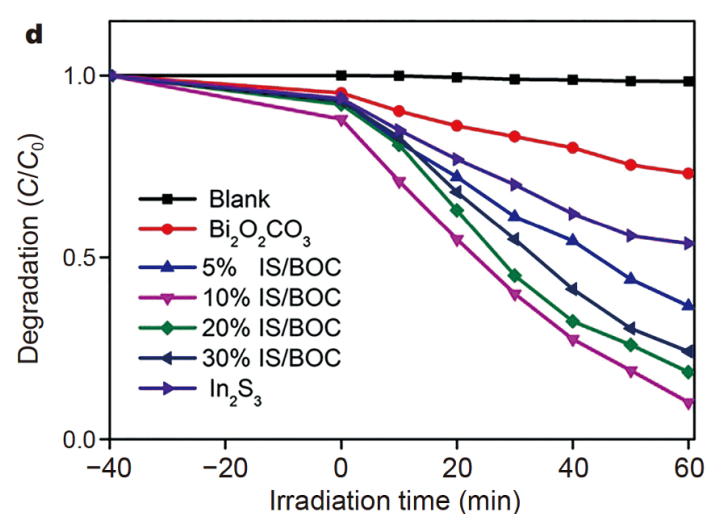

Figure 17 (a) TEM image of $\mathrm{Au} \mathrm{NPs}$ decorated 2D/2D $\mathrm{Bi}_{2} \mathrm{WO}_{6}-\mathrm{TiO}_{2}$ heterostructure. (b) The proposed mechanism for the enhancement of photocatalytic activity over Au NPs decorated $2 \mathrm{D} / 2 \mathrm{D} \mathrm{Bi} \mathrm{WO}_{6}-\mathrm{TiO}_{2}$ heterostructure. SPR: surface plasmon resonance. Reprinted with permission from Ref. [91]. Copyright 2017, Wiley-VCH. (c) Sketch of the 2D/2D $\mathrm{In}_{2} \mathrm{~S}_{3} / \mathrm{Bi}_{2} \mathrm{O}_{2} \mathrm{CO}_{3}$ S-scheme heterojunction. (d) Photocatalytic degradation rates over the $\mathrm{In}_{2} \mathrm{~S}_{3} / \mathrm{Bi}_{2} \mathrm{O}_{2} \mathrm{CO}_{3}$ and other fabricated samples. Reprinted with permission from Ref. [156]. Copyright 2020, Elsevier. 
form 2D/2D p-n type heterojunctions. Under visible light irradiation, the formed $\mathrm{BiOBr} / \mathrm{La}_{2} \mathrm{Ti}_{2} \mathrm{O}_{7} \mathrm{p}$ - $\mathrm{n}$ heterojunction showed an enhanced photocatalytic degradation activity to dye $\mathrm{RhB}$ and phenol. Later, the authors in the same group [46] further synthesized $\mathrm{BiOCl} / \mathrm{La}_{2} \mathrm{Ti}_{2} \mathrm{O}_{7} 2 \mathrm{D} /$ $2 \mathrm{D}$ p-n type heterojunction for photocatalytic degradation of RhB. Two different types of bismuth-contained semiconductors also can be combined to realize the 2D/ $2 \mathrm{D}$ photocatalyst system for environmental remediation, such as $\mathrm{BiOIO}_{3} / \mathrm{BiOI}$ [41], $\mathrm{Bi}_{2} \mathrm{~S}_{3}$ - $\mathrm{BiOCl}$ [201], $\mathrm{Bi}_{4} \mathrm{Ti}_{3} \mathrm{O}_{12} /$ I- $\mathrm{BiOCl}$ [31] and $\mathrm{BiOI} / \mathrm{BiVO}_{4}$ [55].

Besides the aforementioned $\mathrm{g}-\mathrm{C}_{3} \mathrm{~N}_{4}$ based and bismuthcontaining photocatalysts, there are also other advanced $2 \mathrm{D} / 2 \mathrm{D}$ heterojunctions used for environmental remediation, such as $\mathrm{SnNb}_{2} \mathrm{O}_{6} /$ graphene [95], $\mathrm{WO}_{3} / \mathrm{SnNb}_{2} \mathrm{O}_{6}$ [108], $\mathrm{WC} / \mathrm{WO}_{3}$ [202] and $\mathrm{TiO}_{2} / \mathrm{WS}_{2}$ [79].

\section{CONCLUSION AND OUTLOOK}

In the past few years, significant progresses have been made in the synthesis and applications of $2 \mathrm{D} / 2 \mathrm{D}$ heterostructures. The 2D/2D heterostructures possess many superior properties, such as integrating the merits of each $2 \mathrm{D}$ component and dramatically enhanced separation or transfer of charges. Driven by these advantages, 2D/2D heterostructures were deemed as excellent candidates for fundamental photocatalytic research and potential commercial applications. In this comprehensive review paper, we have highlighted the advanced progress of $2 \mathrm{D} / 2 \mathrm{D}$ heterostructure photocatalysts from their general designing strategies to representative photocatalytic applications. As demonstrated by a large amount of literature, the rational design of $2 \mathrm{D} / 2 \mathrm{D}$ heterostructures can significantly boost their photocatalytic activities, mainly attributed to their intrinsic 2D lamellar nature, large contact area and strong interactions. Till now, some representative $2 \mathrm{D} / 2 \mathrm{D}$ heterostructures, such as g- $\mathrm{C}_{3} \mathrm{~N}_{4} /$ $\mathrm{MoS}_{2}, \mathrm{BP} / \mathrm{g}-\mathrm{C}_{3} \mathrm{~N}_{4}, \mathrm{BP} / \mathrm{MoS}_{2} \mathrm{Ti}_{3} \mathrm{C}_{2} / \mathrm{MoS}_{2}, \alpha-\mathrm{Fe}_{2} \mathrm{O}_{3} / \mathrm{GO}$ and g- $\mathrm{C}_{3} \mathrm{~N}_{4} / \mathrm{rGO}$, have shown significant enhancement of photocatalytic performance for hydrogen evolution, $\mathrm{CO}_{2}$ reduction and degradation of pollutants as compared with pristine $2 \mathrm{D}$ materials.

Despite considerable achievements in the design and application of $2 \mathrm{D} / 2 \mathrm{D}$ heterostructure photocatalysts, there are still several challenges ahead in this area. In the first aspect, it is well-known that the large quantity manufacture of photocatalyst materials is of great importance for the potential commercial applications. However, there are few scalable strategies to produce the $2 \mathrm{D} / 2 \mathrm{D}$ heterostructures in large scale with controllable construction. Thus, more effort should be concentrated on the development of low-cost and large-scale production approaches. The second obstacle is the serious agglomeration issue when the different 2D components were coupled together, which would result in loss of the unique structural benefits of the $2 \mathrm{D}$ morphology. In this regard, it encourages developing some strategies to overcome the surface energies of the $2 \mathrm{D} / 2 \mathrm{D}$ heterostructures for better stabilization of freestanding in the $2 \mathrm{D}$ architecture. The third challenge is the lack of study on the effect of the thickness of each 2D layer on the performances of 2D/2D heterostructures. Theoretically, the photocatalytic performances of the $2 \mathrm{D}$ materials are highly dependent on their thickness. For example, the atomic thickness of ultrathin 2D nanomaterials can endow them with high specific surface area, ultimate exposure of their surface atoms and excellent optical transparency, making them exceedingly desirable in the application of photocatalysis. In addition, the thickness of 2D nanomaterials also affects their electronic band structure, which plays a vital role in photocatalytic the application. However, fewer studies have been conducted to investigate the relationship between the thickness of $2 \mathrm{D}$ materials and the photocatalytic performances of their $2 \mathrm{D} / 2 \mathrm{D}$ heterostructures. In view of this point, the relationship between the thicknesses and the photocatalytic performances of $2 \mathrm{D} / 2 \mathrm{D}$ heterostructures can be investigated by controlling the thickness of each $2 \mathrm{D}$ layer. The fourth aspect is a huge lack of deep understanding of the physical and chemical properties as well as fundamental formation mechanisms of the $2 \mathrm{D} / 2 \mathrm{D}$ heterostructure photocatalysts. In this regard, some direct and accurate characterization techniques can be adopted to uncover the intrinsic feature and the real charge transfer pathway over the 2D/2D heterostructures during photocatalytic reactions, such as in-situ Raman spectroscopy, in-situ XPS and in situ electron microscopy. In addition, the rational fundamental calculations and simulations based on density functional theory (DFT) should be also paid much more attention to, because they cannot only provide a better fundamental understanding of the formation as well photocatalytic mechanism but also guide the design of efficient $2 \mathrm{D} / 2 \mathrm{D}$ photocatalysts. Finally, while the researchers explore novel $2 \mathrm{D} / 2 \mathrm{D}$ photocatalysts to meet the requirements of highly efficient photocatalytic performance, one should also pay some attention to that there is plenty of space for developing the existing $2 \mathrm{D} / 2 \mathrm{D}$ heterostructures for potential photocatalytic applications, e.g., hydrogen peroxide production [240].

Benefiting from the rapid development and abundant knowledge accumulated in $2 \mathrm{D}$ materials as well as their 
heterostructures in recent years, one may expect that 2D/ $2 \mathrm{D}$ hybrid photocatalysts would play an important role in solving energy and environmental crisis. It is believed that this comprehensive review will contribute to the further research in the area of $2 \mathrm{D} / 2 \mathrm{D}$ materials or photocatalysis.

\section{Received 5 December 2019; accepted 16 January 2020; published online 1 April 2020}

1 Deng D, Novoselov KS, Fu Q, et al. Catalysis with two-dimensional materials and their heterostructures. Nat Nanotech, 2016, 11: $218-230$

2 Mounet N, Gibertini M, Schwaller P, et al. Two-dimensional materials from high-throughput computational exfoliation of experimentally known compounds. Nat Nanotech, 2018, 13: 246252

3 Liu Y, Huang Y, Duan X. van der Waals integration before and beyond two-dimensional materials. Nature, 2019, 567: 323-333

4 Faraji M, Yousefi M, Yousefzadeh S, et al. Two-dimensional materials in semiconductor photoelectrocatalytic systems for water splitting. Energy Environ Sci, 2019, 12: 59-95

5 Zhu B, Cheng B, Zhang L, et al. Review on DFT calculation of $s$ triazine-based carbon nitride. Carbon Energy, 2019, 1: 32-56

6 Novoselov KS. Electric field effect in atomically thin carbon films. Science, 2004, 306: 666-669

7 Anasori B, Lukatskaya MR, Gogotsi Y. 2D metal carbides and nitrides (MXenes) for energy storage. Nat Rev Mater, 2017, 2: 16098

8 Jin $\mathrm{H}$, Guo C, Liu X, et al. Emerging two-dimensional nanomaterials for electrocatalysis. Chem Rev, 2018, 118: 6337-6408

9 Anichini C, Czepa W, Pakulski D, et al. Chemical sensing with 2D materials. Chem Soc Rev, 2018, 47: 4860-4908

10 Luo B, Liu G, Wang L. Recent advances in 2D materials for photocatalysis. Nanoscale, 2016, 8: 6904-6920

11 Low J, Cao S, Yu J, et al. Two-dimensional layered composite photocatalysts. Chem Commun, 2014, 50: 10768-10777

12 Xiang Q, Cheng B, Yu J. Graphene-based photocatalysts for solarfuel generation. Angew Chem Int Ed, 2015, 54: 11350-11366

13 Ganguly P, Harb M, Cao Z, et al. 2D nanomaterials for photocatalytic hydrogen production. ACS Energy Lett, 2019, 4: 16871709

14 Liu G, Zhen C, Kang Y, et al. Unique physicochemical properties of two-dimensional light absorbers facilitating photocatalysis. Chem Soc Rev, 2018, 47: 6410-6444

15 Li Y, Gao C, Long R, et al. Photocatalyst design based on twodimensional materials. Mater Today Chem, 2019, 11: 197-216

16 Novoselov KS, Mishchenko A, Carvalho A, et al. 2D materials and van der Waals heterostructures. Science, 2016, 353: aac9439

17 Low J, Yu J, Jaroniec M, et al. Heterojunction photocatalysts. Adv Mater, 2017, 29: 1601694

18 Rhodes D, Chae SH, Ribeiro-Palau R, et al. Disorder in van der Waals heterostructures of 2D materials. Nat Mater, 2019, 18: 541549

19 Ren Y, Zeng D, Ong WJ. Interfacial engineering of graphitic carbon nitride $\left(\mathrm{g}-\mathrm{C}_{3} \mathrm{~N}_{4}\right)$-based metal sulfide heterojunction photocatalysts for energy conversion: A review. Chin J Catal, 2019, 40: 289-319

20 Qu Y, Duan X. Progress, challenge and perspective of heterogeneous photocatalysts. Chem Soc Rev, 2013, 42: 2568-2580
21 Wang H, Zhang L, Chen Z, et al. Semiconductor heterojunction photocatalysts: Design, construction, and photocatalytic performances. Chem Soc Rev, 2014, 43: 5234-5244

22 Qi K, Cheng B, Yu J, et al. Review on the improvement of the photocatalytic and antibacterial activities of ZnO. J Alloys Compd, 2017, 727: 792-820

23 Meng A, Zhang L, Cheng B, et al. Dual cocatalysts in $\mathrm{TiO}_{2}$ photocatalysis. Adv Mater, 2019, 31: 1807660

24 Yan J, Verma P, Kuwahara Y, et al. Recent progress on black phosphorus-based materials for photocatalytic water splitting. Small Methods, 2018, 2: 1800212

25 Gan X, Lei D, Wong KY. Two-dimensional layered nanomaterials for visible-light-driven photocatalytic water splitting. Mater Today Energy, 2018, 10: 352-367

26 Su T, Shao Q, Qin Z, et al. Role of interfaces in two-dimensional photocatalyst for water splitting. ACS Catal, 2018, 8: 2253-2276

27 Liu X, Hersam MC. Interface characterization and control of 2D materials and heterostructures. Adv Mater, 2018, 30: 1801586

28 Cao S, Shen $\mathrm{B}$, Tong $\mathrm{T}$, et al. $2 \mathrm{D} / 2 \mathrm{D}$ heterojunction of ultrathin MXene $/ \mathrm{Bi}_{2} \mathrm{WO}_{6}$ nanosheets for improved photocatalytic $\mathrm{CO}_{2}$ reduction. Adv Funct Mater, 2018, 28: 1800136

29 Cheng H, Hou J, Takeda O, et al. A unique Z-scheme 2D/2D nanosheet heterojunction design to harness charge transfer for photocatalysis. J Mater Chem A, 2015, 3: 11006-11013

30 Xia $\mathrm{P}$, Zhu B, Cheng $\mathrm{B}$, et al. $2 \mathrm{D} / 2 \mathrm{D}$ g- $\mathrm{C}_{3} \mathrm{~N}_{4} / \mathrm{MnO}_{2}$ nanocomposite as a direct Z-scheme photocatalyst for enhanced photocatalytic activity. ACS Sustain Chem Eng, 2018, 6: 965-973

31 Qian K, Xia L, Jiang Z, et al. In situ chemical transformation synthesis of $\mathrm{Bi}_{4} \mathrm{Ti}_{3} \mathrm{O}_{12} / \mathrm{I}-\mathrm{BiOCl} 2 \mathrm{D} / 2 \mathrm{D}$ heterojunction systems for water pollution treatment and hydrogen production. Catal Sci Technol, 2017, 7: 3863-3875

32 Gong Y, Lei S, Ye G, et al. Two-step growth of two-dimensional $\mathrm{WSe}_{2} / \mathrm{MoSe}_{2}$ heterostructures. Nano Lett, 2015, 15: 6135-6141

33 Han C, Lei $\mathrm{Y}$, Wang B, et al. In situ-fabricated 2D/2D heterojunctions of ultrathin $\mathrm{SiC} /$ reduced graphene oxide nanosheets for efficient $\mathrm{CO}_{2}$ photoreduction with high $\mathrm{CH}_{4}$ selectivity. ChemSusChem, 2018, 11: 4237-4245

34 Zhang KX, Su H, Wang HH, et al. Atomic-scale mott-schottky heterojunctions of boron nitride monolayer and graphene as metal-free photocatalysts for artificial photosynthesis. Adv Sci, 2018, 5: 1800062

35 Shi J, Li S, Wang F, et al. In situ topotactic formation of 2D/2D direct $\mathrm{Z}$-scheme $\mathrm{Cu}_{2} \mathrm{~S} / \mathrm{Zn}_{0.67} \mathrm{Cd}_{0.33} \mathrm{~S}$ in-plane intergrowth nanosheet heterojunctions for enhanced photocatalytic hydrogen production. Dalton Trans, 2019, 48: 3327-3337

36 Hou Y, Wen Z, Cui S, et al. Constructing 2D porous graphitic $\mathrm{C}_{3} \mathrm{~N}_{4}$ nanosheets/nitrogen-doped graphene/layered $\mathrm{MoS}_{2}$ ternary nanojunction with enhanced photoelectrochemical activity. Adv Mater, 2013, 25: 6291-6297

37 Sun J, Zhang H, Guo LH, et al. Two-dimensional interface engineering of a titania-graphene nanosheet composite for improved photocatalytic activity. ACS Appl Mater Interfaces, 2013, 5: 13035-13041

38 Bera R, Kundu S, Patra A. 2D hybrid nanostructure of reduced graphene oxide-CdS nanosheet for enhanced photocatalysis. ACS Appl Mater Interfaces, 2015, 7: 13251-13259

39 Cho KM, Kim KH, Choi HO, et al. A highly photoactive, visiblelight-driven graphene/2D mesoporous $\mathrm{TiO}_{2}$ photocatalyst. Green Chem, 2015, 17: 3972-3978

40 Yuan YJ, Ye ZJ, Lu HW, et al. Constructing anatase $\mathrm{TiO}_{2}$ na- 
nosheets with exposed (001) facets/layered $\mathrm{MoS}_{2}$ two-dimensional nanojunctions for enhanced solar hydrogen generation. ACS Catal, 2016, 6: 532-541

41 Dong F, Xiong T, Sun Y, et al. Controlling interfacial contact and exposed facets for enhancing photocatalysis via $2 \mathrm{D}-2 \mathrm{D}$ heterostructures. Chem Commun, 2015, 51: 8249-8252

42 Chen W, Liu TY, Huang T, et al. Novel mesoporous P-doped graphitic carbon nitride nanosheets coupled with $\mathrm{ZnIn}_{2} \mathrm{~S}_{4}$ nanosheets as efficient visible light driven heterostructures with remarkably enhanced photo-reduction activity. Nanoscale, 2016, 8: 3711-3719

43 Jia $\mathrm{Y}$, Zhan $\mathrm{S}$, Ma S, et al. Fabrication of $\mathrm{TiO}_{2}-\mathrm{Bi}_{2} \mathrm{WO}_{6}$ binanosheet for enhanced solar photocatalytic disinfection of $E$. coli: Insights on the mechanism. ACS Appl Mater Interfaces, 2016, 8: 6841-6851

44 Wang $\mathrm{Y}$, Xie $\mathrm{Y}$, Sun $\mathrm{H}$, et al. 2D/2D nano-hybrids of $\gamma-\mathrm{MnO}_{2}$ on reduced graphene oxide for catalytic ozonation and coupling peroxymonosulfate activation. J Hazard Mater, 2016, 301: 56-64

45 Ao Y, Wang K, Wang P, et al. Synthesis of novel 2D-2D p-n heterojunction $\mathrm{BiOBr} / \mathrm{La}_{2} \mathrm{Ti}_{2} \mathrm{O}_{7}$ composite photocatalyst with enhanced photocatalytic performance under both UV and visible light irradiation. Appl Catal B-Environ, 2016, 194: 157-168

46 Ao Y, Wang K, Wang P, et al. Fabrication of p-type BiOCl/n-type $\mathrm{La}_{2} \mathrm{Ti}_{2} \mathrm{O}_{7}$ facet-coupling heterostructure with enhanced photocatalytic performance. RSC Adv, 2016, 6: 48599-48609

47 Wang JC, Yao HC, Fan ZY, et al. Indirect Z-scheme BiOI/g- $\mathrm{C}_{3} \mathrm{~N}_{4}$ photocatalysts with enhanced photoreduction $\mathrm{CO}_{2}$ activity under visible light irradiation. ACS Appl Mater Interfaces, 2016, 8: 3765-3775

48 Wang $\mathrm{M}$, Shen $\mathrm{M}$, Zhang L, et al. $2 \mathrm{D}-2 \mathrm{D} \mathrm{MnO}_{2} / \mathrm{g}-\mathrm{C}_{3} \mathrm{~N}_{4}$ heterojunction photocatalyst: $\mathrm{In}$-situ synthesis and enhanced $\mathrm{CO}_{2}$ reduction activity. Carbon, 2017, 120: 23-31

49 Zhang J, Zhang L, Shi Y, et al. Anatase $\mathrm{TiO}_{2}$ nanosheets with coexposed $\{101\}$ and $\{001\}$ facets coupled with ultrathin $\mathrm{SnS}_{2}$ nanosheets as a face-to-face $n-p-n$ dual heterojunction photocatalyst for enhancing photocatalytic activity. Appl Surf Sci, 2017, 420: $839-848$

50 Jo WK, Kumar S, Eslava S, et al. Construction of $\mathrm{Bi}_{2} \mathrm{WO}_{6} / \mathrm{rGO} / \mathrm{g}$ $\mathrm{C}_{3} \mathrm{~N}_{4}$ 2D/2D/2D hybrid Z-scheme heterojunctions with large interfacial contact area for efficient charge separation and highperformance photoreduction of $\mathrm{CO}_{2}$ and $\mathrm{H}_{2} \mathrm{O}$ into solar fuels. Appl Catal B-Environ, 2018, 239: 586-598

51 Kumar S, Kumar A, Kumar A, et al. Highly efficient visible light active 2D-2D nanocomposites of N-ZnO-g- $\mathrm{C}_{3} \mathrm{~N}_{4}$ for photocatalytic degradation of diverse industrial pollutants. ChemistrySelect, 2018, 3: 1919-1932

52 Kumar S, Reddy NL, Kumar A, et al. Two dimensional n-doped $\mathrm{ZnO}$-graphitic carbon nitride nanosheets heterojunctions with enhanced photocatalytic hydrogen evolution. Int J Hydrogen Energy, 2018, 43: 3988-4002

53 Lin $\mathrm{B}$, Li $\mathrm{H}$, An $\mathrm{H}$, et al. Preparation of $2 \mathrm{D} / 2 \mathrm{D} \mathrm{g}-\mathrm{C}_{3} \mathrm{~N}_{4}$ nanosheet@ZnIn $\mathrm{S}_{4}$ nanoleaf heterojunctions with well-designed high-speed charge transfer nanochannels towards high-efficiency photocatalytic hydrogen evolution. Appl Catal B-Environ, 2018, 220: $542-552$

54 Liu J, Li J, Wang L, et al. Synthesis of a novel magnetic $\mathrm{SnNb}_{2} \mathrm{O}_{6}$ / CoFe-LDH 2D/2D heterostructure for the degradation of organic pollutants under visible light irradiation. J Mater Sci, 2018, 54: 172-187

55 Ni S, Zhou $\mathrm{T}$, Zhang $\mathrm{H}$, et al. $\mathrm{BiOI} / \mathrm{BiVO}_{4}$ two-dimensional heteronanostructures for visible-light photocatalytic degradation of rhodamine B. ACS Appl Nano Mater, 2018, 1: 5128-5141

56 Peng C, Wei P, Li X, et al. High efficiency photocatalytic hydrogen production over ternary $\mathrm{Cu} / \mathrm{TiO}_{2} @ \mathrm{Ti}_{3} \mathrm{C}_{2} \mathrm{Tx}$ enabled by low-work-function 2D titanium carbide. Nano Energy, 2018, 53: 97-107

57 Tonda S, Kumar S, Bhardwaj M, et al. g- $\mathrm{C}_{3} \mathrm{~N}_{4} / \mathrm{NiAl}-\mathrm{LDH} 2 \mathrm{D} / 2 \mathrm{D}$ hybrid heterojunction for high-performance photocatalytic reduction of $\mathrm{CO}_{2}$ into renewable fuels. ACS Appl Mater Interfaces, 2018, 10: 2667-2678

58 Wang Q, Wang W, Zhong L, et al. Oxygen vacancy-rich $2 \mathrm{D} / 2 \mathrm{D}$ BiOCl-g- $\mathrm{C}_{3} \mathrm{~N}_{4}$ ultrathin heterostructure nanosheets for enhanced visible-light-driven photocatalytic activity in environmental remediation. Appl Catal B-Environ, 2018, 220: 290-302

59 Wu Y, Wang H, Tu W, et al. Construction of hierarchical 2D-2D $\mathrm{Zn}_{3} \mathrm{In}_{2} \mathrm{~S}_{6} /$ fluorinated polymeric carbon nitride nanosheets photocatalyst for boosting photocatalytic degradation and hydrogen production performance. Appl Catal B-Environ, 2018, 233: 58-69

$60 \mathrm{Xu} \mathrm{X}, \mathrm{Si} \mathrm{Z}$, Liu L, et al. $\mathrm{CoMoS}_{2} / \mathrm{rGO} / \mathrm{C}_{3} \mathrm{~N}_{4}$ ternary heterojunctions catalysts with high photocatalytic activity and stability for hydrogen evolution under visible light irradiation. Appl Surf Sci, 2018, 435: 1296-1306

61 Yuan YJ, Li Z, Wu S, et al. Role of two-dimensional nanointerfaces in enhancing the photocatalytic performance of $2 \mathrm{D}$ $2 \mathrm{D} \mathrm{MoS}_{2} / \mathrm{CdS}$ photocatalysts for $\mathrm{H}_{2}$ production. Chem Eng J, 2018, 350: 335-343

62 Yuan YJ, Yang Y, Li Z, et al. Promoting charge separation in g$\mathrm{C}_{3} \mathrm{~N}_{4} /$ graphene/ $\mathrm{MoS}_{2}$ photocatalysts by two-dimensional nanojunction for enhanced photocatalytic $\mathrm{H}_{2}$ production. ACS Appl Energy Mater, 2018, 1: 1400-1407

63 Zhang K, Zhang Y, Zhang WJ. Ultrathin hexagonal $\mathrm{SnS}_{2}$ nanosheets coupled with tetragonal $\mathrm{CuInS}_{2}$ nanosheets as $2 \mathrm{D} / 2 \mathrm{D}$ heterojunction photocatalysts toward high visible-light photocatalytic activity and stability. Catal Lett, 2018, 148: 1990-2000

64 Liu J, Li J, Bing X, et al. ZnCr-LDH/N-doped graphitic carbonincorporated $g-\mathrm{C}_{3} \mathrm{~N}_{4} 2 \mathrm{D} / 2 \mathrm{D}$ nanosheet heterojunction with enhanced charge transfer for photocatalysis. Mater Res Bull, 2018, 102: $379-390$

65 Shi L, Si W, Wang F, et al. Construction of 2D/2D layered g$\mathrm{C}_{3} \mathrm{~N}_{4} / \mathrm{Bi}_{12} \mathrm{O}_{17} \mathrm{Cl}_{2}$ hybrid material with matched energy band structure and its improved photocatalytic performance. RSC Adv, 2018, 8: 24500-24508

66 Chen W, He ZC, Huang GB, et al. Direct Z-scheme 2D/2D $\mathrm{MnIn}_{2} \mathrm{~S}_{4} / \mathrm{g}-\mathrm{C}_{3} \mathrm{~N}_{4}$ architectures with highly efficient photocatalytic activities towards treatment of pharmaceutical wastewater and hydrogen evolution. Chem Eng J, 2019, 359: 244-253

67 Guan Z, Pan J, Li Q, et al. Boosting visible-light photocatalytic hydrogen evolution with an efficient $\mathrm{CuInS}_{2} / \mathrm{ZnIn}_{2} \mathrm{~S}_{4}$ 2D/2D heterojunction. ACS Sustain Chem Eng, 2019, 7: 7736-7742

68 Guo F, Shi W, Li M, et al. 2D/2D Z-scheme heterojunction of $\mathrm{CuInS}_{2} / \mathrm{g}-\mathrm{C}_{3} \mathrm{~N}_{4}$ for enhanced visible-light-driven photocatalytic activity towards the degradation of tetracycline. Separ Purif Tech, 2019, 210: 608-615

69 Huo Y, Yang Y, Dai K, et al. Construction of 2D/2D porous graphitic $\mathrm{C}_{3} \mathrm{~N}_{4} / \mathrm{SnS}_{2}$ composite as a direct Z-scheme system for efficient visible photocatalytic activity. Appl Surf Sci, 2019, 481: 1260-1269

70 Jiang D, Wen B, Zhang Y, et al. $\mathrm{MoS}_{2} / \mathrm{SnNb}_{2} \mathrm{O}_{6} 2 \mathrm{D} / 2 \mathrm{D}$ nanosheet heterojunctions with enhanced interfacial charge separation for boosting photocatalytic hydrogen evolution. J Colloid Interface 
Sci, 2019, 536: 1-8

71 Jiang R, Lu G, Yan Z, et al. Enhanced photocatalytic activity of a hydrogen bond-assisted 2D/2D Z-scheme $\mathrm{SnNb}_{2} \mathrm{O}_{6} / \mathrm{Bi}_{2} \mathrm{WO}_{6}$ system: Highly efficient separation of photoinduced carriers. J Colloid Interface Sci, 2019, 552: 678-688

72 Jiang $\mathrm{R}, \mathrm{Wu} \mathrm{D}, \mathrm{Lu} \mathrm{G}$, et al. Modified 2D-2D $\mathrm{ZnIn}_{2} \mathrm{~S}_{4} / \mathrm{BiOCl}$ van der Waals heterojunctions with CQDs: Accelerated charge transfer and enhanced photocatalytic activity under vis- and NIRlight. Chemosphere, 2019, 227: 82-92

73 Li J, Zhao Y, Xia M, et al. Highly efficient charge transfer at 2D/ 2D layered $\mathrm{p}-\mathrm{La}_{2} \mathrm{Ti}_{2} \mathrm{O}_{7} / \mathrm{Bi}_{2} \mathrm{WO}_{6}$ contact heterojunctions for upgraded visible-light-driven photocatalysis. Appl Catal B-Environ, 2020, 261: 118244

74 Li M, Zhang Q, Ruan H, et al. An in-situ growth approach to $2 \mathrm{D}$ $\mathrm{MoS}_{2}$-2D PbS heterojunction composites with improved photocatalytic activity. J Solid State Chem, 2019, 270: 98-103

75 Li Y, Yin Z, Ji G, et al. 2D/2D/2D heterojunction of $\mathrm{Ti}_{3} \mathrm{C}_{2}$ MXene/ $\mathrm{MoS}_{2}$ nanosheets/ $\mathrm{TiO}_{2}$ nanosheets with exposed (001) facets toward enhanced photocatalytic hydrogen production activity. Appl Catal B-Environ, 2019, 246: 12-20

76 Liang $\mathrm{X}$, Zhang $\mathrm{Y}$, Li D, et al. $2 \mathrm{D} / 2 \mathrm{D} \mathrm{BiOCl} / \mathrm{K}^{+} \mathrm{Ca}_{2} \mathrm{Nb}_{3} \mathrm{O}_{10}{ }^{-}$heterostructure with $\mathrm{Z}$-scheme charge carrier transfer pathways for tetracycline degradation under simulated solar light. Appl Surf Sci, 2019, 466: 863-873

77 Liu C, Li X, Li J, et al. Fabricated 2D/2D CdIn $\mathrm{S}_{4} / \mathrm{N}-\mathrm{rGO}$ mutiheterostructure photocatalyst for enhanced photocatalytic activity. Carbon, 2019, 152: 565-574

78 Sun L, Zhao Z, Li S, et al. Role of $\mathrm{SnS}_{2}$ in $2 \mathrm{D}-2 \mathrm{D} \mathrm{SnS}_{2} / \mathrm{TiO}_{2}$ nanosheet heterojunctions for photocatalytic hydrogen evolution. ACS Appl Nano Mater, 2019, 2: 2144-2151

$79 \mathrm{Wu} \mathrm{Y}$, Liu Z, Li Y, et al. Construction of 2D-2D TiO 2 nanosheet/ layered $\mathrm{WS}_{2}$ heterojunctions with enhanced visible-light-responsive photocatalytic activity. Chin J Catal, 2019, 40: 60-69

$80 \mathrm{Xu} \mathrm{Q}, \mathrm{Yi}$ H, Lai C, et al. Construction of 2D/2D nano-structured rGO-BWO photocatalysts for efficient tetracycline degradation. Catal Commun, 2019, 124: 113-117

81 Yuan YJ, Shen Z, Wu S, et al. Liquid exfoliation of g- $\mathrm{C}_{3} \mathrm{~N}_{4}$ nanosheets to construct $2 \mathrm{D}-2 \mathrm{D} \mathrm{MoS} / \mathrm{g}-\mathrm{C}_{3} \mathrm{~N}_{4}$ photocatalyst for enhanced photocatalytic $\mathrm{H}_{2}$ production activity. Appl Catal BEnviron, 2019, 246: 120-128

82 Yuan YJ, Wang P, Li Z, et al. The role of bandgap and interface in enhancing photocatalytic $\mathrm{H}_{2}$ generation activity of 2D-2D black phosphorus $/ \mathrm{MoS}_{2}$ photocatalyst. Appl Catal B-Environ, 2019, 242: $1-8$

83 Zhang J, Huang G, Zeng J, et al. $\mathrm{SnS}_{2}$ nanosheets coupled with 2D ultrathin $\mathrm{MoS}_{2}$ nanolayers as face-to-face 2D/2D heterojunction photocatalysts with excellent photocatalytic and photoelectrochemical activities. J Alloys Compd, 2019, 775: 726-735

84 Zou Y, Shi JW, Sun L, et al. Energy-band-controlled $\mathrm{Zn}_{x} \mathrm{Cd}_{1-x} \mathrm{In}_{2} \mathrm{~S}_{4}$ solid solution coupled with $\mathrm{g}-\mathrm{C}_{3} \mathrm{~N}_{4}$ nanosheets as $2 \mathrm{D} / 2 \mathrm{D}$ heterostructure toward efficient photocatalytic $\mathrm{H}_{2}$ evolution. Chem Eng J, 2019, 378: 122192

85 Liu X, Liu Y, Zhang W, et al. In situ self-assembly of 3D hierarchical 2D/2D CdS/g- $\mathrm{C}_{3} \mathrm{~N}_{4}$ hereojunction with excellent photocatalytic performance. Mater Sci Semicond Proc, 2020, 105: 104734

86 Ma S, Xie J, Wen J, et al. Constructing 2D layered hybrid CdS nanosheets/ $\mathrm{MoS}_{2}$ heterojunctions for enhanced visible-light photocatalytic $\mathrm{H}_{2}$ generation. Appl Surf Sci, 2017, 391: 580-591

87 Ran J, Guo W, Wang H, et al. Metal-free 2D/2D phosphorene/g-
$\mathrm{C}_{3} \mathrm{~N}_{4}$ van der Waals heterojunction for highly enhanced visiblelight photocatalytic $\mathrm{H}_{2}$ production. Adv Mater, 2018, 30: 1800128

$88 \mathrm{Xu} \mathrm{Y}$, You Y, Huang $\mathrm{H}$, et al. $\mathrm{Bi}_{4} \mathrm{NbO}_{8} \mathrm{Cl}\{001\}$ nanosheets coupled with $\mathrm{g}-\mathrm{C}_{3} \mathrm{~N}_{4}$ as $2 \mathrm{D} / 2 \mathrm{D}$ heterojunction for photocatalytic degradation and $\mathrm{CO}_{2}$ reduction. J Hazard Mater, 2020, 381: 121159

89 Liu $\mathrm{X}$, Zhou $\mathrm{Z}$, Han $\mathrm{D}$, et al. Interface engineered 2D/2D $\mathrm{Ni}(\mathrm{OH})_{2} / \mathrm{Bi}_{4} \mathrm{Ti}_{3} \mathrm{O}_{12}$ nanocomposites with higher charge transfer towards improving photocatalytic activity. J Alloys Compd, 2020, 818: 152530

90 Tan P, Zhu A, Qiao L, et al. Constructing a direct Z-scheme photocatalytic system based on $2 \mathrm{D} / 2 \mathrm{D} \quad \mathrm{WO}_{3} / \mathrm{ZnIn}_{2} \mathrm{~S}_{4}$ nanocomposite for efficient hydrogen evolution under visible light. Inorg Chem Front, 2019, 6: 929-939

91 Yuan L, Weng B, Colmenares JC, et al. Multichannel charge transfer and mechanistic insight in metal decorated 2D-2D $\mathrm{Bi}_{2} \mathrm{WO}_{6}-\mathrm{TiO}_{2}$ cascade with enhanced photocatalytic performance. Small, 2017, 13: 1702253

$92 \mathrm{Xu}$ Q, Zhu B, Jiang C, et al. Constructing 2D/2D Fe $\mathrm{O}_{3} / \mathrm{g}^{-} \mathrm{C}_{3} \mathrm{~N}_{4}$ direct Z-scheme photocatalysts with enhanced $\mathrm{H}_{2}$ generation performance. Sol RRL, 2018, 2: 1800006

93 Jiang $\mathrm{D}$, Wang $\mathrm{T}, \mathrm{Xu} \mathrm{Q}$, et al. Perovskite oxide ultrathin nanosheets/g- $\mathrm{C}_{3} \mathrm{~N}_{4} 2 \mathrm{D}-2 \mathrm{D}$ heterojunction photocatalysts with significantly enhanced photocatalytic activity towards the photodegradation of tetracycline. Appl Catal B-Environ, 2017, 201: $617-628$

94 Ma X, Jiang D, Xiao P, et al. 2D/2D heterojunctions of $\mathrm{WO}_{3}$ nanosheet $/ \mathrm{K}^{+} \mathrm{Ca}_{2} \mathrm{Nb}_{3} \mathrm{O}_{10}{ }^{-}$ultrathin nanosheet with improved charge separation efficiency for significantly boosting photocatalysis. Catal Sci Technol, 2017, 7: 3481-3491

95 Yuan L, Yang MQ, Xu YJ. Tuning the surface charge of graphene for self-assembly synthesis of a $\mathrm{SnNb}_{2} \mathrm{O}_{6}$ nanosheet-graphene (2D-2D) nanocomposite with enhanced visible light photoactivity. Nanoscale, 2014, 6: 6335-6345

96 Ong WJ, Tan LL, Chai SP, et al. Surface charge modification via protonation of graphitic carbon nitride $\left(\mathrm{g}-\mathrm{C}_{3} \mathrm{~N}_{4}\right)$ for electrostatic self-assembly construction of $2 \mathrm{D} / 2 \mathrm{D}$ reduced graphene oxide $(\mathrm{rGO}) / \mathrm{g}-\mathrm{C}_{3} \mathrm{~N}_{4}$ nanostructures toward enhanced photocatalytic reduction of carbon dioxide to methane. Nano Energy, 2015, 13: $757-770$

97 Zhang Z, Huang J, Zhang M, et al. Ultrathin hexagonal $\mathrm{SnS}_{2}$ nanosheets coupled with g- $\mathrm{C}_{3} \mathrm{~N}_{4}$ nanosheets as $2 \mathrm{D} / 2 \mathrm{D}$ heterojunction photocatalysts toward high photocatalytic activity. Appl Catal B-Environ, 2015, 163: 298-305

98 Chen J, Xu X, Li T, et al. Toward high performance 2D/2D hybrid photocatalyst by electrostatic assembly of rationally modified carbon nitride on reduced graphene oxide. Sci Rep, 2016, 6: 37318

99 Huang $\mathrm{H}$, Xiao $\mathrm{K}$, Tian $\mathrm{N}$, et al. Dual visible-light active components containing self-doped $\mathrm{Bi}_{2} \mathrm{O}_{2} \mathrm{Co}_{3} / g-\mathrm{C}_{3} \mathrm{~N}_{4}$ 2D-2D heterojunction with enhanced visible-light-driven photocatalytic activity. Colloids Surfs A-Physicochem Eng Aspects, 2016, 511: 64-72

$100 \mathrm{Li} \mathrm{J}$, Liu E, Ma Y, et al. Synthesis of $\mathrm{MoS}_{2} / \mathrm{g}-\mathrm{C}_{3} \mathrm{~N}_{4}$ nanosheets as 2D heterojunction photocatalysts with enhanced visible light activity. Appl Surf Sci, 2016, 364: 694-702

101 Yan J, Chen Z, Ji H, et al. Construction of a 2D graphene-like $\mathrm{MoS}_{2} / \mathrm{C}_{3} \mathrm{~N}_{4}$ heterojunction with enhanced visible-light photocatalytic activity and photoelectrochemical activity. Chem Eur J, 2016, 22: 4764-4773

102 Zhang Z, Jiang D, Li D, et al. Construction of $\mathrm{SnNb}_{2} \mathrm{O}_{6}$ na- 
nosheet/g- $\mathrm{C}_{3} \mathrm{~N}_{4}$ nanosheet two-dimensional heterostructures with improved photocatalytic activity: Synergistic effect and mechanism insight. Appl Catal B-Environ, 2016, 183: 113-123

103 Wan W, Yu S, Dong F, et al. Efficient $\mathrm{C}_{3} \mathrm{~N}_{4} /$ graphene oxide macroscopic aerogel visible-light photocatalyst. J Mater Chem A, 2016, 4: 7823-7829

$104 \mathrm{Hu}$ S, Zhang W, Bai J, et al. Construction of a $2 \mathrm{D} / 2 \mathrm{D}$ g- $\mathrm{C}_{3} \mathrm{~N}_{4} / \mathrm{rGO}$ hybrid heterojunction catalyst with outstanding charge separation ability and nitrogen photofixation performance via a surface protonation process. RSC Adv, 2016, 6: 25695-25702

105 Cai X, Zhang J, Fujitsuka M, et al. Graphitic- $\mathrm{C}_{3} \mathrm{~N}_{4}$ hybridized Ndoped $\mathrm{La}_{2} \mathrm{Ti}_{2} \mathrm{O}_{7}$ two-dimensional layered composites as efficient visible-light-driven photocatalyst. Appl Catal B-Environ, 2017, 202: 191-198

$106 \mathrm{Ma} \mathrm{X}, \mathrm{Ma} \mathrm{W}$, Jiang $\mathrm{D}$, et al. Construction of novel $\mathrm{WO}_{3} / \mathrm{SnNb}_{2} \mathrm{O}_{6}$ hybrid nanosheet heterojunctions as efficient $\mathrm{Z}$-scheme photocatalysts for pollutant degradation. J Colloid Interface Sci, 2017, 506: 93-101

107 Sultana S, Mansingh S, Parida KM. Facile synthesis of $\mathrm{CeO}_{2}$ nanosheets decorated upon BiOI microplate: A surface oxygen vacancy promoted Z-scheme-based 2D-2D nanocomposite photocatalyst with enhanced photocatalytic activity. J Phys Chem C, 2017, 122: 808-819

108 Zhu M, Kim S, Mao L, et al. Metal-free photocatalyst for $\mathrm{H}_{2}$ evolution in visible to near-infrared region: black phosphorus/ graphitic carbon nitride. J Am Chem Soc, 2017, 139: 13234-13242

109 Gong Y, Quan X, Yu H, et al. Enhanced photocatalytic performance of a two-dimensional $\mathrm{BiOIO}_{3} / \mathrm{g}-\mathrm{C}_{3} \mathrm{~N}_{4}$ heterostructured composite with a Z-scheme configuration. Appl Catal B-Environ, 2018, 237: 947-956

110 Xie L, Ni J, Tang B, et al. A self-assembled 2D/2D-type protonated carbon nitride-modified graphene oxide nanocomposite with improved photocatalytic activity. Appl Surf Sci, 2018, 434: 456-463

111 Guo W, Fan K, Zhang J, et al. 2D/2D Z-scheme $\mathrm{Bi}_{2} \mathrm{WO}_{6} /$ porous$\mathrm{g}^{-} \mathrm{C}_{3} \mathrm{~N}_{4}$ with synergy of adsorption and visible-light-driven photodegradation. Appl Surf Sci, 2018, 447: 125-134

112 Li X, Wang X, Zhu J, et al. Fabrication of two-dimensional $\mathrm{Ni}_{2} \mathrm{P} /$ $\mathrm{ZnIn}_{2} \mathrm{~S}_{4}$ heterostructures for enhanced photocatalytic hydrogen evolution. Chem Eng J, 2018, 353: 15-24

113 Zhang K, Fujitsuka M, Du Y, et al. 2D/2D heterostructured CdS/ $\mathrm{WS}_{2}$ with efficient charge separation improving $\mathrm{H}_{2}$ evolution under visible light irradiation. ACS Appl Mater Interfaces, 2018, 10: 20458-20466

114 Ji H, Fei T, Zhang L, et al. Synergistic effects of $\mathrm{MoO}_{2}$ nanosheets and graphene-like $\mathrm{C}_{3} \mathrm{~N}_{4}$ for highly improved visible light photocatalytic activities. Appl Surf Sci, 2018, 457: 1142-1150

115 Shi X, Fujitsuka M, Kim S, et al. Faster electron injection and more active sites for efficient photocatalytic $\mathrm{H}_{2}$ evolution in g$\mathrm{C}_{3} \mathrm{~N}_{4} / \mathrm{MoS}_{2}$ hybrid. Small, 2018, 14: 1703277

$116 \mathrm{Fu} \mathrm{J}, \mathrm{Xu} \mathrm{Q}$, Low J, et al. Ultrathin $2 \mathrm{D} / 2 \mathrm{D} \mathrm{WO}_{3} / \mathrm{g}-\mathrm{C}_{3} \mathrm{~N}_{4}$ stepscheme $\mathrm{H}_{2}$-production photocatalyst. Appl Catal B-Environ, 2019, 243: 556-565

117 Huang L, Han B, Huang X, et al. Ultrathin 2D/2D ZnIn ${ }_{2} \mathrm{~S}_{4} / \mathrm{MoS}_{2}$ hybrids for boosted photocatalytic hydrogen evolution under visible light. J Alloys Compd, 2019, 798: 553-559

118 Lin $\mathrm{P}$, Shen J, $\mathrm{Yu}$ X, et al. Construction of $\mathrm{Ti}_{3} \mathrm{C}_{2}$ MXene/O-doped $\mathrm{g}^{-} \mathrm{C}_{3} \mathrm{~N}_{4}$ 2D-2D schottky-junction for enhanced photocatalytic hydrogen evolution. Ceramics Int, 2019, 45: 24656-24663

119 Su T, Hood ZD, Naguib M, et al. 2D/2D heterojunction of $\mathrm{Ti}_{3} \mathrm{C}_{2} /$ g- $\mathrm{C}_{3} \mathrm{~N}_{4}$ nanosheets for enhanced photocatalytic hydrogen evolution. Nanoscale, 2019, 11: 8138-8149

120 Sun Z, Yu Z, Liu Y, et al. Construction of $2 \mathrm{D} / 2 \mathrm{D} \mathrm{BiVO}_{4} / \mathrm{g}-\mathrm{C}_{3} \mathrm{~N}_{4}$ nanosheet heterostructures with improved photocatalytic activity. J Colloid Interface Sci, 2019, 533: 251-258

121 Yang H, Cao R, Sun P, et al. Constructing electrostatic self-assembled 2D/2D ultra-thin $\mathrm{ZnIn}_{2} \mathrm{~S}_{4}$ /protonated g- $\mathrm{C}_{3} \mathrm{~N}_{4}$ heterojunctions for excellent photocatalytic performance under visible light. Appl Catal B-Environ, 2019, 256: 117862

122 Bafaqeer A, Tahir M, Amin NAS. Well-designed $\mathrm{ZnV}_{2} \mathrm{O}_{6} / \mathrm{g}^{-} \mathrm{C}_{3} \mathrm{~N}_{4}$ $2 \mathrm{D} / 2 \mathrm{D}$ nanosheets heterojunction with faster charges separation via $\mathrm{pCN}$ as mediator towards enhanced photocatalytic reduction of $\mathrm{CO}_{2}$ to fuels. Appl Catal B-Environ, 2019, 242: 312-326

123 Song $\mathrm{Y}, \mathrm{Gu} J$, Xia K, et al. Construction of $2 \mathrm{D} \mathrm{SnS} \mathrm{S}_{2} / \mathrm{g}-\mathrm{C}_{3} \mathrm{~N}_{4} \mathrm{Z}$ scheme composite with superior visible-light photocatalytic performance. Appl Surf Sci, 2019, 467-468: 56-64

124 Zhang X, Deng J, Yan J, et al. Cryo-mediated liquid-phase exfoliated $2 \mathrm{D}$ BP coupled with $2 \mathrm{D} \mathrm{C}_{3} \mathrm{~N}_{4}$ to photodegradate organic pollutants and simultaneously generate hydrogen. Appl Surf Sci, 2019, 490: 117-123

125 Kong XY, Lee WQ, Mohamed AR, et al. Effective steering of charge flow through synergistic inducing oxygen vacancy defects and $\mathrm{p}$-n heterojunctions in $2 \mathrm{D} / 2 \mathrm{D}$ surface-engineered $\mathrm{Bi}_{2} \mathrm{WO}_{6} /$ BiOI cascade: Towards superior photocatalytic $\mathrm{CO}_{2}$ reduction activity. Chem Eng J, 2019, 372: 1183-1193

126 Jacobson MZ, Colella WG, Golden DM. Cleaning the air and improving health with hydrogen fuel-cell vehicles. Science, 2005, 308: 1901-1905

127 Sharma S, Ghoshal SK. Hydrogen the future transportation fuel: From production to applications. Renew Sustain Energy Rev, 2015, 43: 1151-1158

128 Mohanty P, Pant KK, Mittal R. Hydrogen generation from biomass materials: Challenges and opportunities. WIREs Energy Environ, 2015, 4: 139-155

129 Yang Y, Fei H, Ruan G, et al. Porous cobalt-based thin film as a bifunctional catalyst for hydrogen generation and oxygen generation. Adv Mater, 2015, 27: 3175-3180

130 Chen X, Shen S, Guo L, et al. Semiconductor-based photocatalytic hydrogen generation. Chem Rev, 2010, 110: 6503-6570

131 Low J, Yu J, Ho W. Graphene-based photocatalysts for $\mathrm{CO}_{2}$ reduction to solar fuel. J Phys Chem Lett, 2015, 6: 4244-4251

$132 \mathrm{Yu} \mathrm{W}$, Zhang S, Chen J, et al. Biomimetic Z-scheme photocatalyst with a tandem solid-state electron flow catalyzing $\mathrm{H}_{2}$ evolution. J Mater Chem A, 2018, 6: 15668-15674

133 Ahmad H, Kamarudin SK, Minggu LJ, et al. Hydrogen from photo-catalytic water splitting process: A review. Renew Sustain Energy Rev, 2015, 43: 599-610

134 Xiang Q, Yu J. Graphene-based photocatalysts for hydrogen generation. J Phys Chem Lett, 2013, 4: 753-759

135 Park S, Chang WJ, Lee CW, et al. Photocatalytic hydrogen generation from hydriodic acid using methylammonium lead iodide in dynamic equilibrium with aqueous solution. Nat Energy, 2017, 2: 16185

136 Xia $\mathrm{P}$, Liu M, Cheng B, et al. Dopamine modified g- $\mathrm{C}_{3} \mathrm{~N}_{4}$ and its enhanced visible-light photocatalytic $\mathrm{H}_{2}$-production activity. ACS Sustain Chem Eng, 2018, 6: 8945-8953

137 Zhao Z, Xing Y, Li H, et al. Constructing CdS/Cd/doped $\mathrm{TiO}_{2} \mathrm{Z}$ scheme type visible light photocatalyst for $\mathrm{H}_{2}$ production. Sci China Mater, 2018, 61: 851-860

138 Wang Y, Vogel A, Sachs M, et al. Current understanding and 
challenges of solar-driven hydrogen generation using polymeric photocatalysts. Nat Energy, 2019, 4: 746-760

139 Tang Y, Zhou P, Chao Y, et al. Face-to-face engineering of ultrathin Pd nanosheets on amorphous carbon nitride for efficient photocatalytic hydrogen production. Sci China Mater, 2019, 62: 351-358

140 Ren D, Shen R, Jiang Z, et al. Highly efficient visible-light photocatalytic $\mathrm{H}_{2}$ evolution over $2 \mathrm{D}-2 \mathrm{D} \mathrm{CdS} / \mathrm{Cu}_{7} \mathrm{~S}_{4}$ layered heterojunctions. Chin J Catal, 2020, 41: 31-40

$141 \mathrm{Gu} \mathrm{W,} \mathrm{Lu} \mathrm{F,} \mathrm{Wang} \mathrm{C,} \mathrm{et} \mathrm{al.} \mathrm{Face-to-face} \mathrm{interfacial} \mathrm{assembly} \mathrm{of}$ ultrathin $\mathrm{g}_{-} \mathrm{C}_{3} \mathrm{~N}_{4}$ and anatase $\mathrm{TiO}_{2}$ nanosheets for enhanced solar photocatalytic activity. ACS Appl Mater Interfaces, 2017, 9: 28674-28684

142 Zhong R, Zhang Z, Yi H, et al. Covalently bonded 2D/2D O-g$\mathrm{C}_{3} \mathrm{~N}_{4} / \mathrm{TiO}_{2}$ heterojunction for enhanced visible-light photocatalytic hydrogen evolution. Appl Catal B-Environ, 2018, 237: 1130-1138

143 Wang K, Li Y, Li J, et al. Boosting interfacial charge separation of $\mathrm{Ba}_{5} \mathrm{Nb}_{4} \mathrm{O}_{15} / g-\mathrm{C}_{3} \mathrm{~N}_{4}$ photocatalysts by $2 \mathrm{D} / 2 \mathrm{D}$ nanojunction towards efficient visible-light driven $\mathrm{H}_{2}$ generation. Appl Catal BEnviron, 2020, 263: 117730

144 Hua $\mathrm{E}$, Jin $\mathrm{S}$, Wang $\mathrm{X}$, et al. Ultrathin 2D type-II p-n heterojunctions $\mathrm{La}_{2} \mathrm{Ti}_{2} \mathrm{O}_{7} / \mathrm{In}_{2} \mathrm{~S}_{3}$ with efficient charge separations and photocatalytic hydrogen evolution under visible light illumination. Appl Catal B-Environ, 2019, 245: 733-742

145 Qi K, Cheng B, Yu J, et al. A review on $\mathrm{TiO}_{2}$-based Z-scheme photocatalysts. Chin J Catal, 2017, 38: 1936-1955

$146 \mathrm{Yu} \mathrm{W}$, Chen J, Shang T, et al. Direct Z-scheme g- $\mathrm{C}_{3} \mathrm{~N}_{4} / \mathrm{WO}_{3}$ photocatalyst with atomically defined junction for $\mathrm{H}_{2}$ production. Appl Catal B-Environ, 2017, 219: 693-704

147 Jourshabani M, Shariatinia Z, Badiei A. High efficiency visiblelight-driven $\mathrm{Fe}_{2} \mathrm{O}_{3-x} \mathrm{~S}_{x} / \mathrm{S}$-doped $\mathrm{g}-\mathrm{C}_{3} \mathrm{~N}_{4}$ heterojunction photocatalysts: Direct Z-scheme mechanism. J Mater Sci Tech, 2018, 34: $1511-1525$

$148 \mathrm{Xu} \mathrm{Q}$, Zhang L, Yu J, et al. Direct Z-scheme photocatalysts: Principles, synthesis, and applications. Mater Today, 2018, 21: 1042-1063

149 Di T, Xu Q, Ho WK, et al. Review on metal sulphide-based Zscheme photocatalysts. ChemCatChem, 2019, 11: 1394-1411

150 She $\mathrm{X}, \mathrm{Wu} \mathrm{J}, \mathrm{Xu} \mathrm{H}$, et al. High efficiency photocatalytic water splitting using $2 \mathrm{D} a-\mathrm{Fe}_{2} \mathrm{O}_{3} / g-\mathrm{C}_{3} \mathrm{~N}_{4}$ Z-scheme catalysts. Adv Energy Mater, 2017, 7: 1700025

151 Liu D, Zhang S, Wang J, et al. Direct Z-scheme 2D/2D photocatalyst based on ultrathin $\mathrm{g}-\mathrm{C}_{3} \mathrm{~N}_{4}$ and $\mathrm{WO}_{3}$ nanosheets for efficient visible-light-driven $\mathrm{H}_{2}$ generation. ACS Appl Mater Interfaces, 2019, 11: 27913-27923

152 Huang Y, Liu Y, Zhu D, et al. Mediator-free Z-scheme photocatalytic system based on ultrathin CdS nanosheets for efficient hydrogen evolution. J Mater Chem A, 2016, 4: 13626-13635

$153 \mathrm{Hu}$ J, Chen D, Mo Z, et al. Z-scheme 2D/2D heterojunction of black phosphorus/monolayer $\mathrm{Bi}_{2} \mathrm{WO}_{6}$ nanosheets with enhanced photocatalytic activities. Angew Chem, 2019, 131: 2095-2099

154 Ge $\mathrm{H}, \mathrm{Xu}$ F, Cheng B, et al. S-scheme heterojunction $\mathrm{TiO}_{2} / \mathrm{CdS}$ nanocomposite nanofiber as $\mathrm{H}_{2}$-production photocatalyst. ChemCatChem, 2019, 11: 6301-6309

155 He F, Meng A, Cheng B, et al. Enhanced photocatalytic $\mathrm{H}_{2}$-production activity of $\mathrm{WO}_{3} / \mathrm{TiO}_{2}$ step-scheme heterojunction by graphene modification. Chin J Catal, 2020, 41: 9-20

156 Fan $\mathrm{H}$, Zhou H, Li W, et al. Facile fabrication of $2 \mathrm{D} / 2 \mathrm{D}$ stepscheme $\mathrm{In}_{2} \mathrm{~S}_{3} / \mathrm{Bi}_{2} \mathrm{O}_{2} \mathrm{Co}_{3}$ heterojunction towards enhanced photo- catalytic activity. Appl Surf Sci, 2020, 504: 144351

157 Wang J, Zhang Q, Deng F, et al. Rapid toxicity elimination of organic pollutants by the photocatalysis of environment-friendly and magnetically recoverable step-scheme $\mathrm{SnFe}_{2} \mathrm{O}_{4} / \mathrm{ZnFe}_{2} \mathrm{O}_{4}$ nano-heterojunctions. Chem Eng J, 2020, 379: 122264

158 Luo J, Lin Z, Zhao Y, et al. The embedded $\mathrm{CuInS}_{2}$ into hollowconcave carbon nitride for photocatalytic $\mathrm{H}_{2} \mathrm{O}$ splitting into $\mathrm{H}_{2}$ with S-scheme principle. Chin J Catal, 2020, 41: 122-130

159 Ren D, Zhang W, Ding Y, et al. In situ fabrication of robust cocatalyst-free CdS/g- $\mathrm{C}_{3} \mathrm{~N}_{4}$ 2D-2D step-scheme heterojunctions for highly active $\mathrm{H}_{2}$ evolution. Sol RRL, 2020, 1900423

160 Yuan YJ, Chen D, Zhong J, et al. Interface engineering of a noblemetal-free $2 \mathrm{D}-2 \mathrm{D} \mathrm{MoS} / \mathrm{Cu}-\mathrm{ZnIn}_{2} \mathrm{~S}_{4}$ photocatalyst for enhanced photocatalytic $\mathrm{H}_{2}$ production. J Mater Chem A, 2017, 5: 1577115779

161 Shi R, Waterhouse GIN, Zhang T. Recent progress in photocatalytic $\mathrm{CO}_{2}$ reduction over perovskite oxides. Sol RRL, 2017, 1: 1700126

162 Chen Y, Jia G, Hu Y, et al. Two-dimensional nanomaterials for photocatalytic $\mathrm{CO}_{2}$ reduction to solar fuels. Sustain Energy Fuels, 2017, 1: 1875-1898

163 Loiudice A, Lobaccaro P, Kamali EA, et al. Tailoring copper nanocrystals towards $\mathrm{C}_{2}$ products in electrochemical $\mathrm{CO}_{2}$ reduction. Angew Chem Int Ed, 2016, 55: 5789-5792

164 Gonzales JN, Matson MM, Atsumi S. Nonphotosynthetic biological $\mathrm{CO}_{2}$ reduction. Biochemistry, 2018, 58: 1470-1477

165 Chueh WC, Falter C, Abbott M, et al. High-flux solar-driven thermochemical dissociation of $\mathrm{CO}_{2}$ and $\mathrm{H}_{2} \mathrm{O}$ using nonstoichiometric ceria. Science, 2010, 330: 1797-1801

166 Low J, Cheng B, Yu J, et al. Carbon-based two-dimensional layered materials for photocatalytic $\mathrm{CO}_{2}$ reduction to solar fuels. Energy Storage Mater, 2016, 3: 24-35

167 Bie $\mathrm{C}$, Zhu B, Xu F, et al. In situ grown monolayer N-doped graphene on CdS hollow spheres with seamless contact for photocatalytic $\mathrm{CO}_{2}$ reduction. Adv Mater, 2019, 31: 1902868

168 Zeng S, Kar P, Thakur UK, et al. A review on photocatalytic $\mathrm{CO}_{2}$ reduction using perovskite oxide nanomaterials. Nanotechnology, 2018, 29: 052001

169 Inoue T, Fujishima A, Konishi S, et al. Photoelectrocatalytic reduction of carbon dioxide in aqueous suspensions of semiconductor powders. Nature, 1979, 277: 637-638

170 Navalón S, Dhakshinamoorthy A, Alvaro M, et al. Photocatalytic $\mathrm{CO}_{2}$ reduction using non-titanium metal oxides and sulfides. ChemSusChem, 2013, 6: 562-577

171 Di T, Zhu B, Cheng B, et al. A direct Z-scheme g- $\mathrm{C}_{3} \mathrm{~N}_{4} / \mathrm{SnS}_{2}$ photocatalyst with superior visible-light $\mathrm{CO}_{2}$ reduction performance. J Catal, 2017, 352: 532-541

172 Low J, Cheng B, Yu J. Surface modification and enhanced photocatalytic $\mathrm{CO}_{2}$ reduction performance of $\mathrm{TiO}_{2}$ : A review. Appl Surf Sci, 2017, 392: 658-686

173 Han C, Li J, Ma Z, et al. Black phosphorus quantum dot $/ \mathrm{g}-\mathrm{C}_{3} \mathrm{~N}_{4}$ composites for enhanced $\mathrm{CO}_{2}$ photoreduction to $\mathrm{CO}$. Sci China Mater, 2018, 61: 1159-1166

174 Zhang N, Long R, Gao C, et al. Recent progress on advanced design for photoelectrochemical reduction of $\mathrm{CO}_{2}$ to fuels. Sci China Mater, 2018, 61: 771-805

175 Wang S, Wang Y, Zang S-, et al. Hierarchical hollow heterostructures for photocatalytic $\mathrm{CO}_{2}$ reduction and water splitting. Small Methods, 2020, 4: 1900586

176 Low J, Dai B, Tong T, et al. In situ irradiated X-ray photoelectron 
spectroscopy investigation on a direct Z-scheme $\mathrm{TiO}_{2} / \mathrm{CdS}$ composite film photocatalyst. Adv Mater, 2019, 31: 1802981

177 Dai K, Bergot A, Liang C, et al. Environmental issues associated with wind energy-A review. Renew Energy, 2015, 75: 911-921

178 Matzek LW, Carter KE. Activated persulfate for organic chemical degradation: A review. Chemosphere, 2016, 151: 178-188

179 Shi X, Wang C, Ma Y, et al. Template-free microwave-assisted synthesis of FeTi coordination complex yolk-shell microspheres for superior catalytic removal of arsenic and chemical degradation of methylene blue from polluted water. Powder Tech, 2019, 356: 726-734

180 Gao C, Zhang W, Li H, et al. Controllable fabrication of mesoporous $\mathrm{MgO}$ with various morphologies and their absorption performance for toxic pollutants in water. Cryst Growth Des, 2008, 8: 3785-3790

181 Le Borgne S, Paniagua D, Vazquez-Duhalt R. Biodegradation of organic pollutants by halophilic bacteria and archaea. J Mol Microbiol Biotechnol, 2008, 15: 74-92

182 Wang CC, Li JR, Lv XL, et al. Photocatalytic organic pollutants degradation in metal-organic frameworks. Energy Environ Sci, 2014, 7: 2831-2867

183 Fan Y, Ma W, Han D, et al. Convenient recycling of 3D AgX/ graphene aerogels $(\mathrm{X}=\mathrm{Br}, \mathrm{Cl})$ for efficient photocatalytic degradation of water pollutants. Adv Mater, 2015, 27: 3767-3773

184 Liu C, Ren X, Lin F, et al. Structure of the $\mathrm{Au}_{23-x} \mathrm{Ag}_{x}(\mathrm{~S}-\mathrm{Adm})_{15}$ nanocluster and its application for photocatalytic degradation of organic pollutants. Angew Chem Int Ed, 2019, 58: 11335-11339

185 Tanveer M, Wu Y, Qadeer MA, et al. Atypical $\mathrm{BiOCl} / \mathrm{Bi}_{2} \mathrm{~S}_{3}$ hetero-structures exhibiting remarkable photo-catalyst response. Sci China Mater, 2018, 61: 101-111

186 Yan Q, Huang GF, Li DF, et al. Facile synthesis and superior photocatalytic and electrocatalytic performances of porous Bdoped $\mathrm{g}-\mathrm{C}_{3} \mathrm{~N}_{4}$ nanosheets. J Mater Sci Tech, 2018, 34: 2515-2520

187 Liang Q, Liu X, Zeng G, et al. Surfactant-assisted synthesis of photocatalysts: Mechanism, synthesis, recent advances and environmental application. Chem Eng J, 2019, 372: 429-451

188 Bhatkhande DS, Pangarkar VG, Beenackers AACM. Photocatalytic degradation for environmental applications-A review. J Chem Technol Biotechnol, 2002, 77: 102-116

189 Fan Y, Hu G, Yu S, et al. Recent advances in $\mathrm{TiO}_{2}$ nanoarrays/ graphene for water treatment and energy conversion/storage. Sci China Mater, 2019, 62: 325-340

190 Li X, Xiong J, Xu Y, et al. Defect-assisted surface modification enhances the visible light photocatalytic performance of $\mathrm{g}$ $\mathrm{C}_{3} \mathrm{~N}_{4} @ \mathrm{C}-\mathrm{TiO}_{2}$ direct Z-scheme heterojunctions. Chin J Catal, 2019, 40: 424-433

191 Wang $\mathrm{K}$, Liu B, Li J, et al. In-situ synthesis of $\mathrm{TiO}_{2}$ nanostructures on $\mathrm{Ti}$ foil for enhanced and stable photocatalytic performance. J Mater Sci Tech, 2019, 35: 615-622

192 Gaya UI, Abdullah AH. Heterogeneous photocatalytic degradation of organic contaminants over titanium dioxide: A review of fundamentals, progress and problems. J PhotoChem PhotoBiol C-PhotoChem Rev, 2008, 9: 1-12

193 Ahmed S, Rasul MG, Martens WN, et al. Heterogeneous photocatalytic degradation of phenols in wastewater: A review on current status and developments. Desalination, 2010, 261: 3-18

194 Zhuang H, Xu W, Lin L, et al. Construction of one dimensional $\mathrm{ZnWO}_{4} @ \mathrm{SnWO}_{4}$ core-shell heterostructure for boosted photocatalytic performance. J Mater Sci Tech, 2019, 35: 2312-2318

195 Che H, Che G, Zhou P, et al. Yeast-derived carbon sphere as a bridge of charge carriers towards to enhanced photocatalytic activity of $2 \mathrm{D} / 2 \mathrm{D} \mathrm{Cu} \mathrm{Cu}_{4} / \mathrm{g}-\mathrm{C}_{3} \mathrm{~N}_{4}$ heterojunction. J Colloid Interface Sci, 2019, 546: 262-275

196 Wang J, Tang L, Zeng G, et al. Atomic scale g- $\mathrm{C}_{3} \mathrm{~N}_{4} / \mathrm{Bi}_{2} \mathrm{WO}_{6} 2 \mathrm{D} /$ $2 \mathrm{D}$ heterojunction with enhanced photocatalytic degradation of ibuprofen under visible light irradiation. Appl Catal B-Environ, 2017, 209: 285-294

197 Che $\mathrm{H}$, Che $\mathrm{G}$, Dong $\mathrm{H}$, et al. Fabrication of $\mathrm{Z}$-scheme $\mathrm{Bi}_{3} \mathrm{O}_{4} \mathrm{Cl} / \mathrm{g}$ $\mathrm{C}_{3} \mathrm{~N}_{4}$ 2D/2D heterojunctions with enhanced interfacial charge separation and photocatalytic degradation various organic pollutants activity. Appl Surf Sci, 2018, 455: 705-716

198 Li Y, Zhang $\mathrm{H}$, Liu $\mathrm{P}$, et al. Cross-linked $\mathrm{g}-\mathrm{C}_{3} \mathrm{~N}_{4} / \mathrm{rGO}$ nanocomposites with tunable band structure and enhanced visible light photocatalytic activity. Small, 2013, 9: 3336-3344

199 Wang $\mathrm{X}$, Wang $\mathrm{H}, \mathrm{Yu} \mathrm{K}$, et al. Immobilization of 2D/2D structured $\mathrm{g}-\mathrm{C}_{3} \mathrm{~N}_{4}$ nanosheet/reduced graphene oxide hybrids on $3 \mathrm{D}$ nickel foam and its photocatalytic performance. Mater Res Bull, 2018, 97: 306-313

200 Zheng Y, Yu Z, Ou H, et al. Black phosphorus and polymeric carbon nitride heterostructure for photoinduced molecular oxygen activation. Adv Funct Mater, 2018, 28: 1705407

201 Xu J, Yang J, Zhang P, et al. Preparation of $2 \mathrm{D}$ square-like $\mathrm{Bi}_{2} \mathrm{~S}_{3}$ $\mathrm{BiOCl}$ heterostructures with enhanced visible light-driven photocatalytic performance for dye pollutant degradation. Water Sci Eng, 2017, 10: 334-339

202 Wang SL, Zhu Y, Luo X, et al. 2D WC/ $\mathrm{WO}_{3}$ heterogeneous hybrid for photocatalytic decomposition of organic compounds with vis-NIR light. Adv Funct Mater, 2018, 28: 1705357

203 Hou Y, Laursen AB, Zhang J, et al. Layered nanojunctions for hydrogen-evolution catalysis. Angew Chem Int Ed, 2013, 52: 3621-3625

204 Kumar S, Reddy NL, Kushwaha HS, et al. Efficient electron transfer across a $\mathrm{ZnO}-\mathrm{MoS}_{2}$-reduced graphene oxide heterojunction for enhanced sunlight-driven photocatalytic hydrogen evolution. ChemSusChem, 2017, 10: 3588-3603

205 Xing W, Li C, Wang Y, et al. A novel 2D/2D carbonized poly(furfural alcohol)/g- $\mathrm{C}_{3} \mathrm{~N}_{4}$ nanocomposites with enhanced charge carrier separation for photocatalytic $\mathrm{H}_{2}$ evolution. Carbon, 2017, 115: 486-492

206 Yang MQ, Xu YJ, Lu W, et al. Self-surface charge exfoliation and electrostatically coordinated 2D hetero-layered hybrids. Nat Commun, 2017, 8: 14224

207 Yang MQ, Dan J, Pennycook SJ, et al. Ultrathin nickel boron oxide nanosheets assembled vertically on graphene: A new hybrid 2D material for enhanced photo/electro-catalysis. Mater Horiz, 2017, 4: 885-894

208 Yuan YJ, Chen D, Zhong J, et al. Construction of a noble-metalfree photocatalytic $\mathrm{H}_{2}$ evolution system using $\mathrm{MoS}_{2}$ /reduced graphene oxide catalyst and zinc porphyrin photosensitizer. J Phys Chem C, 2017, 121: 24452-24462

209 Li N, Zhou J, Sheng Z, et al. Molten salt-mediated formation of $\mathrm{g}$ $\mathrm{C}_{3} \mathrm{~N}_{4}-\mathrm{MoS}_{2}$ for visible-light-driven photocatalytic hydrogen evolution. Appl Surf Sci, 2018, 430: 218-224

210 Shi J, Li S, Wang F, et al. $2 \mathrm{D} / 2 \mathrm{D}$ g- $\mathrm{C}_{3} \mathrm{~N}_{4} / \mathrm{MgFe}$ mmo nanosheet heterojunctions with enhanced visible-light photocatalytic $\mathrm{H}_{2}$ production. J Alloys Compd, 2018, 769: 611-619

211 Wan J, Pu C, Wang $\mathrm{R}$, et al. A facile dissolution strategy facilitated by $\mathrm{H}_{2} \mathrm{SO}_{4}$ to fabricate a $2 \mathrm{D}$ metal-free $g-\mathrm{C}_{3} \mathrm{~N}_{4} / \mathrm{rGO}$ heterojunction for efficient photocatalytic $\mathrm{H}_{2}$ production. Int $\mathrm{J}$ Hydrogen Energy, 2018, 43: 7007-7019 
212 Wang XJ, Tian X, Sun YJ, et al. Enhanced Schottky effect of a 2D2D CoP/g- $\mathrm{C}_{3} \mathrm{~N}_{4}$ interface for boosting photocatalytic $\mathrm{H}_{2}$ evolution. Nanoscale, 2018, 10: 12315-12321

213 Yin XL, Li LL, Li DC, et al. One-pot synthesis of CdS-MoS $/$ RGO$\mathrm{E}$ nano-heterostructure with well-defined interfaces for efficient photocatalytic $\mathrm{H}_{2}$ evolution. Int J Hydrogen Energy, 2018, 43: 20382-20391

214 Zhu M, Sun Z, Fujitsuka M, et al. Z-scheme photocatalytic water splitting on a $2 \mathrm{D}$ heterostructure of black phosphorus/bismuth vanadate using visible light. Angew Chem Int Ed, 2018, 57: 21602164

215 Zou Y, Shi JW, Ma D, et al. WS 2 /graphitic carbon nitride heterojunction nanosheets decorated with CdS quantum dots for photocatalytic hydrogen production. ChemSusChem, 2018, 11: 1187-1197

216 Li W, Wang L, Zhang Q, et al. Fabrication of an ultrathin 2D/2D $\mathrm{C}_{3} \mathrm{~N}_{4} / \mathrm{MoS}_{2}$ heterojunction photocatalyst with enhanced photocatalytic performance. J Alloys Compd, 2019, 808: 151681

217 Liang Y, Shang R, Lu J, et al. 2D MOFs enriched g- $\mathrm{C}_{3} \mathrm{~N}_{4}$ nanosheets for highly efficient charge separation and photocatalytic hydrogen evolution from water. Int J Hydrogen Energy, 2019, 44: 2797-2810

218 Yang Z, Wei J, Zeng G, et al. A review on strategies to LDH-based materials to improve adsorption capacity and photoreduction efficiency for $\mathrm{CO}_{2}$. Coord Chem Rev, 2019, 386: 154-182

219 Gai Q, Zheng X, Liu W, et al. 2D-2D heterostructured CaS-CoP photocatalysts for efficient $\mathrm{H}_{2}$ evolution under visible light irradiation. Int J Hydrogen Energy, 2019, 44: 27412-27420

220 Zhou X, Zhu Y, Gao Q, et al. Modified graphitic carbon nitride nanosheets for efficient photocatalytic hydrogen evolution. ChemSusChem, 2019, 12: 4996-5006

221 Shi W, Li M, Huang X, et al. Facile synthesis of $2 \mathrm{D} / 2 \mathrm{D} \mathrm{Co}_{3}\left(\mathrm{PO}_{4}\right)_{2} /$ g- $\mathrm{C}_{3} \mathrm{~N}_{4}$ heterojunction for highly photocatalytic overall water splitting under visible light. Chem Eng J, 2020, 382: 122960

222 Jia J, Sun W, Zhang Q, et al. Inter-plane heterojunctions within 2D/2D FeSe $2 / g-\mathrm{C}_{3} \mathrm{~N}_{4}$ nanosheet semiconductors for photocatalytic hydrogen generation. Appl Catal B-Environ, 2019, 261: 118249

223 Bafaqeer A, Tahir M, Amin NAS. Synergistic effects of 2D/2D $\mathrm{ZnV}_{2} \mathrm{O}_{6} / \mathrm{rGO}$ nanosheets heterojunction for stable and high performance photo-induced $\mathrm{CO}_{2}$ reduction to solar fuels. Chem Eng J, 2018, 334: 2142-2153

224 Jiang $\mathrm{Z}$, Wan W, Li H, et al. A hierarchical Z-scheme $\alpha-\mathrm{Fe}_{2} \mathrm{O}_{3} / \mathrm{g}$ $\mathrm{C}_{3} \mathrm{~N}_{4}$ hybrid for enhanced photocatalytic $\mathrm{CO}_{2}$ reduction. Adv Mater, 2018, 30: 1706108

$225 \mathrm{Mu} \mathrm{Q}$, Zhu W, Li X, et al. Electrostatic charge transfer for boosting the photocatalytic $\mathrm{CO}_{2}$ reduction on metal centers of $2 \mathrm{D}$ $\mathrm{MOF} / \mathrm{rGO}$ heterostructure. Appl Catal B-Environ, 2020, 262: 118144

226 Han S, Hu L, Liang Z, et al. One-step hydrothermal synthesis of 2D hexagonal nanoplates of $a-\mathrm{Fe}_{2} \mathrm{O}_{3} /$ graphene composites with enhanced photocatalytic activity. Adv Funct Mater, 2014, 24: 5719-5727

227 Tian N, Zhang Y, Liu C, et al. g- $\mathrm{C}_{3} \mathrm{~N}_{4} / \mathrm{Bi}_{4} \mathrm{O}_{5} \mathrm{I}_{2} 2 \mathrm{D}-2 \mathrm{D}$ heterojunctional nanosheets with enhanced visible-light photocatalytic activity. RSC Adv, 2016, 6: 10895-10903 g- $\mathrm{C}_{3} \mathrm{~N}_{4}$ nanosheet-supported $\mathrm{MgIn}_{2} \mathrm{~S}_{4}$ nanoplates as visible-lightactive heterostructures for enhanced photocatalytic activity. J Catal, 2017, 349: 8-18

229 Ji M, Di J, Ge Y, et al. 2D-2D stacking of graphene-like g- $\mathrm{C}_{3} \mathrm{~N}_{4} /$ ultrathin $\mathrm{Bi}_{4} \mathrm{O}_{5} \mathrm{Br}_{2}$ with matched energy band structure towards antibiotic removal. Appl Surf Sci, 2017, 413: 372-380

230 Aliaga J, Cifuentes N, González G, et al. Enhancement photocatalytic activity of the heterojunction of two-dimensional hybrid semiconductors $\mathrm{ZnO} / \mathrm{V}_{2} \mathrm{O}_{5}$. Catalysts, 2018, 8: 374

231 Chaudhuri RG, Chaturvedi A, Iype E. Visible light active 2D $\mathrm{C}_{3} \mathrm{~N}_{4}$-CdS hetero-junction photocatalyst for effective removal of azo dye by photodegradation. Mater Res Express, 2018, 5: 036202

232 Liu C, Zhu H, Zhu Y, et al. Ordered layered n-doped $\mathrm{KTiNbO}_{5} / \mathrm{g}$ $\mathrm{C}_{3} \mathrm{~N}_{4}$ heterojunction with enhanced visible light photocatalytic activity. Appl Catal B-Environ, 2018, 228: 54-63

233 Xie $\mathrm{T}$, Liu $\mathrm{Y}$, Wang $\mathrm{H}$, et al. Layered $\mathrm{MoSe}_{2} / \mathrm{Bi}_{2} \mathrm{WO}_{6}$ composite with $\mathrm{p}-\mathrm{n}$ heterojunctions as a promising visible-light induced photocatalyst. Appl Surf Sci, 2018, 444: 320-329

234 Zhang J, Liu G, Liu S. 2D/2D feocl/graphite oxide heterojunction with enhanced catalytic performance as a photo-fenton catalyst. New J Chem, 2018, 42: 6896-6902

235 Chen J, Xiao X, Wang Y, et al. Ag nanoparticles decorated $\mathrm{WO}_{3} /$ g- $\mathrm{C}_{3} \mathrm{~N}_{4} 2 \mathrm{D} / 2 \mathrm{D}$ heterostructure with enhanced photocatalytic activity for organic pollutants degradation. Appl Surf Sci, 2019, 467468: $1000-1010$

236 Jo WK, Tonda S. Novel CoAl-LDH/g- $\mathrm{C}_{3} \mathrm{~N}_{4} / \mathrm{RGO}$ ternary heterojunction with notable $2 \mathrm{D} / 2 \mathrm{D} / 2 \mathrm{D}$ configuration for highly efficient visible-light-induced photocatalytic elimination of dye and antibiotic pollutants. J Hazard Mater, 2019, 368: 778-787

237 Le S, Li W, Wang Y, et al. Carbon dots sensitized 2D-2D heterojunction of $\mathrm{BiVO}_{4} / \mathrm{Bi}_{3} \mathrm{TaO}_{7}$ for visible light photocatalytic removal towards the broad-spectrum antibiotics. J Hazard Mater, 2019, 376: 1-11

238 Liang C, Niu CG, Zhang L, et al. Construction of 2D heterojunction system with enhanced photocatalytic performance: Plasmonic $\mathrm{Bi}$ and reduced graphene oxide co-modified $\mathrm{Bi}_{5} \mathrm{O}_{7} \mathrm{I}$ with high-speed charge transfer channels. J Hazard Mater, 2019, 361: 245-258

239 Vidyasagar D, Gupta A, Balapure A, et al. $2 \mathrm{D} / 2 \mathrm{D} \mathrm{Wg}-\mathrm{C}_{3} \mathrm{~N}_{4} / \mathrm{g}$ $\mathrm{C}_{3} \mathrm{~N}_{4}$ composite as "adsorb and shuttle" model photocatalyst for pollution mitigation. J Photochem Photobiol A-Chem, 2019, 370: 117-126

240 Hou H, Zeng X, Zhang X. Production of hydrogen peroxide through photocatalytic processes: A critical review of recent advances. Angew Chem Int Ed, 2019

Acknowledgements This work was financially supported by the Australia Research Council (ARC DP 180102062), and the National Natural Science Foundation of China (51602163).

Author contributions Zhang X proposed the topic and outline of the manuscript; Hou $\mathrm{H}$ collected the related information and drafted the manuscript; Zeng X gave some valuable comments.

Conflict of interest The authors declare that they have no conflict of interest. 


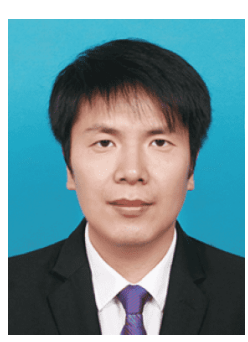

Huilin Hou received his $\mathrm{PhD}$ degree from Taiyuan University of Technology in 2015. He works in Ningbo University of Technology (NBUT), and is currently an associate professor of the Institute of Materials in NBUT. From 2018 to 2019 , he worked at Monash University as a visiting scholar. His research interest focuses on solar photocatalysis.

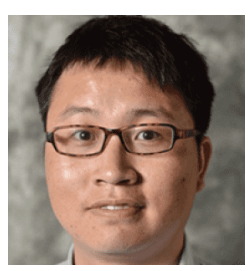

Xiangkang Zeng obtained his $\mathrm{PhD}$ degree in 2017 at Monash University, Australia, under the supervision of Prof. Xiwang Zhang. He then moved to the Hong Kong University of Science and Technology for one-year postdoctoral work. In November 2018, he came back to Australia and is currently a postdoctoral research fellow at Prof. Xiwang Zhang's group. His current research focuses on the development of $2 \mathrm{D}$ photocatalysts.

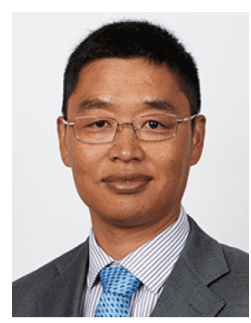

Xiwang Zhang is a professor in the Department of Chemical Engineering at Monash University, and the director of ARC Research Hub for Energy-efficient Separation. His research interests focus on membrane and advanced oxidation technologies for various applications. Prof. Zhang was the receipt of the prestigious Australian Research Fellowship and Larkins Fellowship.

\section{二维/二维异质结光催化剂: 合理设计与能源和环 境应用}

侯慧林 ${ }^{1,2}$, 曾祥康 ${ }^{2}$, 张西旺 ${ }^{2 *}$

摘要 近年来, 二维/二维异质结纳米材料在光催化领域引起了广 泛的研究. 二维/二维异质结材料在高效光催化剂的应用研究上具 有许多独特优点, 主要包括良好的尺寸设计, 较大的界面接触面积, 有机集合了各二维组分的优点, 同时异质结的构筑能够快速促进 光生电荷的分离和转移. 本文首先介绍了二维/二维异质结构光催 化剂所形成界面的一些基本原理内容, 并总结了目前二维/二维异 质结构光催化剂的一般合成策略, 包括原位生长和非原位外组装. 随后着重介绍了二维/二维异质结光催化剂在产氢、二氧化碳还原 和污染物去除等方面的应用的最新研究进展. 最后, 对二维/二维异 质结光催化剂的发展前景进行了展望. 